\title{
lamentat

\section{Mining Natural Products for Macrocycles to Drug Difficult Targets}

Fabio Begnini," Vasanthanathan Poongavanam," Björn Over, " Marie Castaldo, Stefan Geschwindner, Patrik Johansson, Mohit Tyagi, Christian Tyrchan, Lisa Wissler, Peter Sjö, Stefan Schiesser,* and Jan Kihlberg*

Cite This: J. Med. Chem. 2021, 64, 1054-1072

Read Online

ACCESS

|山ll Metrics \& More

Article Recommendations

Supporting Information

ABSTRACT: Lead generation for difficult-to-drug targets that have large, featureless, and highly lipophilic or highly polar and/or flexible binding sites is highly challenging. Here, we describe how cores of macrocyclic natural products can serve as a high-quality in silico screening library that provides leads for difficult-to-drug targets. Two iterative rounds of docking of a carefully selected set of natural-product-derived cores led to the discovery of an uncharged macrocyclic inhibitor of the Keap1-Nrf2 proteinprotein interaction, a particularly challenging target due to its highly polar binding site. The inhibitor displays cellular efficacy

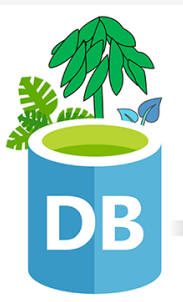

Natural Products

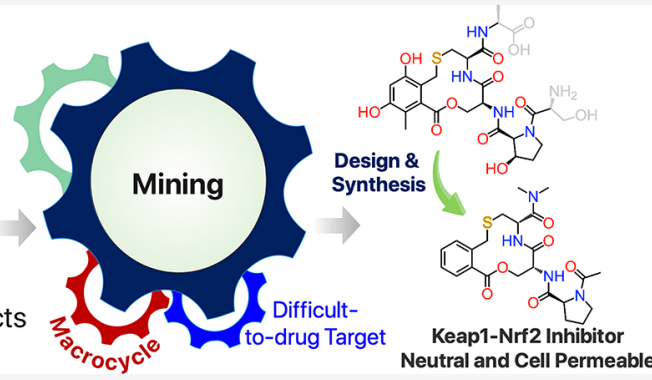
and is well-positioned for further optimization based on the structure of its complex with Keap1 and synthetic access. We believe that our work will spur interest in using macrocyclic cores for in silico-based lead generation and also inspire the design of future macrocycle screening collections.

\section{INTRODUCTION}

More than half of all targets predicted to be involved in human disease are considered to be difficult to modulate with traditional small-molecule drugs that obey Lipinski's rule of 5 (Ro5). ${ }^{1}$ Protein-protein interactions (PPIs), proteases, some kinases as well as transferases and isomerases are important examples., ${ }^{2,3}$ These difficult-to-drug targets often have binding sites that are large, featureless, highly lipophilic or highly polar, and/or flexible. ${ }^{2-4}$ Finding orally bioavailable drugs that reach intracellular difficult-to-drug targets is a daunting task that often requires discovery of ligands in the uncharted chemical space beyond the Ro5 (bRo5). ${ }^{2,5,6}$ Macrocycles ${ }^{7}$ are enriched among oral drugs in the bRo5 space ${ }^{5}$ because they offer superior binding to targets that have large and featureless binding sites as compared to traditional small molecules. ${ }^{2,8}$ In addition, macrocyclization may improve plasma stability, cell permeability, and oral absorption. ${ }^{9}$ However, macrocycles are often under-represented in screening collections, limiting their use for lead generation. For instance, the AstraZeneca collection contains less than 17000 macrocycles out of a total of more than 3.8 million compounds.

Despite a growing number of bRo5 compounds entering clinical trials, lead generation for difficult-to-drug targets has not been systematically investigated and is challenging as the chemical space expands dramatically with increasing molecular size. ${ }^{10}$ Natural products are an important source of drugs, ${ }^{11}$ and we hypothesized that the cores of macrocycles may be seen as nature's privileged substructures and could play a similar role in lead generation for difficult-to-drug targets as fragments do for traditional targets. ${ }^{12,13}$ A collection of lead-like macrocyclic cores would then be useful for in silico screening against targets such as PPIs, with subsequent optimization providing novel leads. In addition, such a collection could inspire the design of natural-product-like macrocycle screening libraries.

Here, we report how two sets of macrocyclic cores that may be used to discover leads for difficult-to-drug targets were generated by a comprehensive investigation of macrocyclic natural product chemical space. Docking of the smaller and more lead-like set into the highly charged binding site for Nrf2 on Keap1 identified the core of cyclothialidine (1) as a potential inhibitor of Keap1. A second round of docking studies using structurally simplified analogues of the core, followed by synthesis, identified a weak inhibitor of the Keap1-Nrf2 PPI. Optimization of the hit via synthesis of an additional nine compounds led to an inhibitor (14), which has a potency in the low $\mu \mathrm{M}$ range and displays cellular activity. The crystal structure of the complex of Keap1 and $\mathbf{1 4}$ reveals how the uncharged macrocycle 14 binds to the charged binding site of Keap 1 and provides a platform for its further optimization.

Received: September 8, 2020

Published: December 18, 2020 


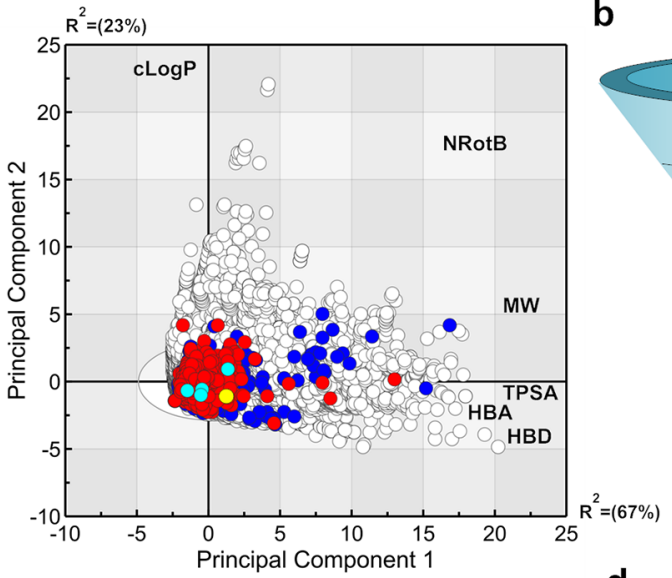

b $\quad>150,000$ Natural Products

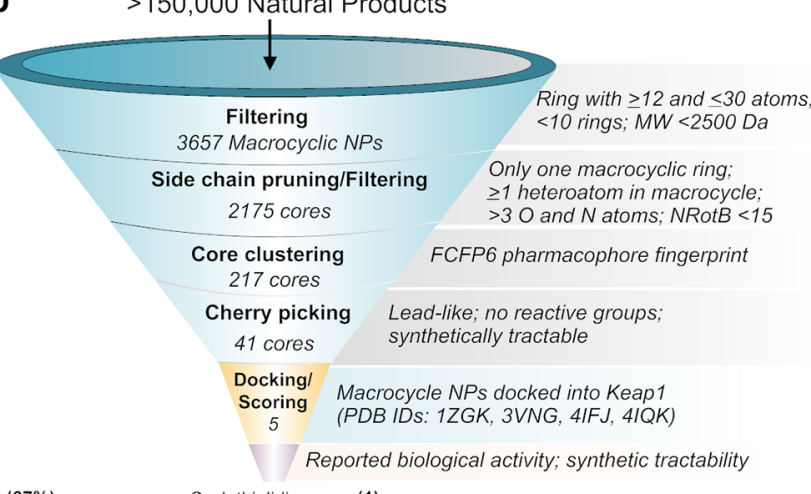

Cyclothialidine core (1)

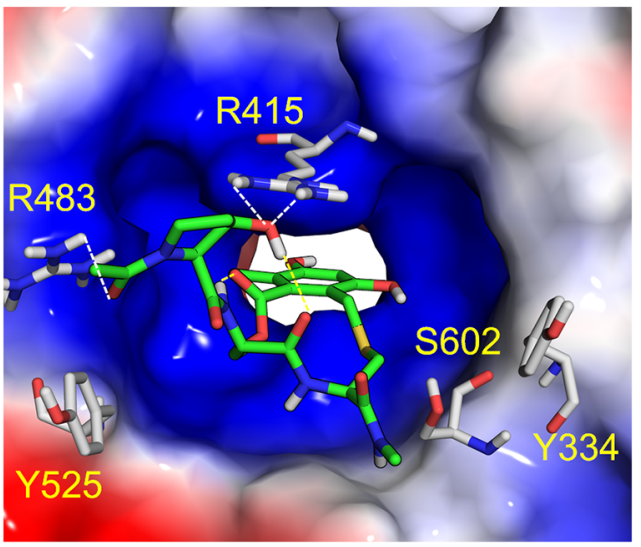

Figure 1. Mining the Dictionary of Natural Products for macrocycle cores. (a) Principal component analysis comparing the chemical space of all compounds in the Dictionary of Natural Products ${ }^{14}$ (white circles) to that of orally and parenterally administered drugs from DrugBank ${ }^{16}$ (red and blue circles, respectively). The first two principal components explain $90 \%$ of the variance in the data set $\left(R^{2}=0.90, Q^{2}=0.70\right)$. The positions of the core of cyclothialidine (1, yellow circle) and macrocycle cores $\mathbf{2}-\mathbf{5}$ (circles in cyan) are indicated. The contribution of descriptors used to characterize the drugs to each of the principal components is indicated by their position on each of the axes. Descriptors: cLogP, calculated lipophilicity; HBA, hydrogen-bond acceptor; HBD, hydrogen-bond donor; MW, molecular weight; NRotB, number of rotatable bonds; TPSA, topological polar surface area. (b) Funnel describing how the Dictionary of Natural Products was filtered and clustered to give one large set of 217 macrocycle cores and one smaller set of 41 lead-like cores. The identification of the core of cyclothialidine (1) by docking of the lead-like cores into the binding site of Keap1 is also indicated. (c) Structures of the five top-ranked macrocycles obtained by docking into Keap1. The bonds of the docked cores are in black and their heteroatoms are colored, while side chains that were pruned from the original natural product have bonds and heteroatoms in gray. (d) Pose obtained from docking of the core of cyclothialidine (1) into the binding site of Keap1 (PDB ID: 4IQK), in which the substituted phenylene group protrudes into the Kelch channel. Key residues in the Keap1 binding site are highlighted.

\section{RESULTS}

Mining the Dictionary of Natural Products. The Dictionary of Natural Products (DNP) ${ }^{14}$ contains $>150000$ compounds that cover a vast chemical space. This space includes that of approved oral and parenteral drugs but also extends far beyond (Figures 1a and S1). Therefore, we considered the DNP as an attractive source for the identification of macrocyclic cores as promising starting points for difficult-to-drug targets. First, duplicates and natural products containing known toxicophores and/or very reactive groups were removed from the DNP using the HTS filter implemented in Pipeline Pilot, ${ }^{12}$ which removes several functionalities including acyl halides, anhydrides, diazo groups, and hydrazides. Then, the remaining compounds were filtered to contain at least one macrocyclic ring, not more than ten smaller rings, and to have a molecular weight $(\mathrm{MW})<2500$ $\mathrm{Da}$ (Figures $1 \mathrm{~b}$ and S2). This removed all nonmacrocycles and large macrocycles, e.g., of polysaccharidic or polypeptidic nature, which are unlikely to reach intracellular targets and provided a data set of 3657 macrocycles. The side chains of this set were pruned to attachment points consisting of at least two heavy atoms or functional groups connected to the macrocycle using the first two steps of the fragment generation algorithm reported previously. ${ }^{12}$ In this way, fragmentation of the macrocycle ring was avoided. The resulting extended Murcko scaffolds, ${ }^{15}$ herein denoted macrocycle cores, were filtered to contain at least a total of three oxygen and nitrogen atoms and $<15$ rotatable bonds, thereby removing highly lipophilic and/or flexible cores unlikely to be developable into drugs. Close to 2200 structurally different cores were identified in this macrocycle collection, which were clustered into a set of 217 representative cores. Cores that contained a few remaining reactive groups (i.e., disulfides, peroxides, and alkyl halides), had an overwhelming synthetic complexity, or that were oligomeric (oligopeptides and oligosaccharides) were removed by visual inspection. This provided a smaller set of 41 more lead-like cores that to a large extent adhered to Lipinski's rule of 5 (Table S1 and Figure S3). As molecular weight and complexity may be expected to increase during optimization, they were judged to be suitable starting points for development into cell-permeable inhibitors of difficult-to-drug targets. This set of cores originates from 35 natural products for which the absolute stereochemistry 
has been reported and from three natural products for which only the relative stereochemistry is known, which were included as enantiomeric pairs. The two sets of cores constitute unique in silico collections that can be used in efforts to find novel starting points in drug discovery (Supporting Excel Sheet). The structural complexity varies between the cores in the two sets; some can be readily synthesized, while simplified analogues may be more attractive starting points for other macrocycles.

Novel Class of Inhibitors of the Keap1-Nrf2 PPI. We chose the PPI between Kelch-like ECH-associated protein 1 (Keap1) and nuclear factor erythroid 2-related factor 2 (Nrf2), to evaluate our sets of macrocyclic cores. The Keap1-Nrf2 system is an important cellular response mechanism for oxidative stress, which is involved in many chronic, age-related, or inflammatory diseases. ${ }^{17}$ Inhibitors of this PPI are of major interest for drug discovery as Nrf2 controls the expression of several cytoprotective genes but is negatively regulated through complexation with Keap1, leading to its ubiquitination and degradation. The Keap1-Nrf2 PPI consists of a highly polar pocket on Keap1, which binds the peptide motif DEETGE on $\mathrm{Nrf2}$, and other negatively charged ligands, with high affinity. ${ }^{18,19}$ This renders it challenging to find cell-permeable and orally absorbed ligands that reach and inhibit the Keap1Nrf2 PPI, ${ }^{19-21}$ just as for other targets that have polar binding sites. $^{22,23}$ Targeting the Keap1-Nrf2 PPI in CNS disorders is particularly challenging. ${ }^{19,20}$ We hypothesize that macrocycles, due to their conformational restriction, may provide an optimal fit in the polar binding site of Keap1. Thus, they may be ideal for the discovery of novel uncharged inhibitors with improved cell permeability and oral absorption as compared to current Keap 1 inhibitors, the majority of which are acidic. ${ }^{20,21}$

The binding site on Keap1 for Nrf2 shows a certain degree of conformational mobility. ${ }^{21}$ In particular, the side chain of Arg415 adopts its conformation so that the binding site is more open in the apo form and more closed when bound to smallmolecule inhibitors. To avoid bias toward a particular binding site conformation when docking the macrocyclic cores, we selected four high-resolution crystal structures of Keap1 that showed variation in the binding site: two with a bound smallmolecule ligand (PDB ID: $4 \mathrm{IQK}^{24}$ and $3 \mathrm{VNG}$ ) and two apo structures (PDB ID: 4IFJ and $1 \mathrm{ZGK}^{25}$ ) (Figure S4). Docking of the 41 cores of the lead-like set into the binding site was then performed using flexible docking in Glide. ${ }^{26}$ The ten cores that docked best into each Keap1 structure were identified by their GlideScores (Table S2). Then, the cores that had docked into three or four of the Keap1 structures were identified from the combined top-ten lists and selected as potential hits as we anticipated them to be more likely to bind to Keap 1 than cores that docked well into only one or two crystal structures (Table S3). Interestingly, the resulting top-five macrocycles displayed a large structural diversity (Figure 1c). Inspection of the docked poses revealed that the core of cyclothialidine (1), which docked well into all four Keap1 structures, was able to bind deep in the binding site and reached into the Kelch channel (Figure 1d). The cores of piperazinomycin (2), 8-ethoxy-3-oxo-1,2-dehydroretrorsine (3), numismine (4), and iriomoteolide 3a (5), which docked well into only three of the Keap1 structures, bound closer to the periphery, providing fewer opportunities for interactions with Keap1 (Figure S5). To the best of our knowledge, the natural product precursors of cores $1-5$ have not been reported to bind to Keap1. However, cytotoxicity has been reported for the natural product precursor of core 5, which may make 5 less suitable for development of an inhibitor of the
Keap1-Nrf2 PPI (Table S4). No synthetic routes have been reported for $\mathbf{3}$ and $\mathbf{4}$, the routes to $\mathbf{2}$ and $\mathbf{5}$ are long, ${ }^{27,28}$ while a route suitable for synthesis of analogues has been reported for the core of $1 .{ }^{29}$ Overall, these considerations revealed the cyclothialidine core as the most promising starting point for discovery of a novel Keapl inhibitor.

Induced-fit docking suggested that simplified analogues of cyclothialidine core, such as stereoisomers 6-9 where the hydroxyl and methyl groups of the phenylene group and the hydroxyl of the proline have been removed (Figure 2a), would also bind to Keap1 (Figure S6). In addition, the docking also indicated that 8 and 9 were more likely to bind stronger than 6 and 7. Satisfactorily, 9 was found to inhibit the binding of Keap1 to an immobilized peptide derived from $\mathrm{Nrf2}\left(K_{\mathrm{D}}=237 \mu \mathrm{M}\right)$ in a surface plasmon resonance (SPR)-based inhibition in solution assay (ISA) ${ }^{30}$ (Figure 2a). Inversion of the stereochemistry of the L-proline moiety to give $\mathbf{1 0}$ led to a reduced binding affinity that revealed its importance for inhibition of Keap1, while hydroxylation of L-proline (11) maintained the $K_{D}$ (Figures 2a and S7). Importantly, replacement of the methyl ester with different amides $(12-15)$ provided dimethylamide 14 , which is close to 2 orders of magnitude more potent than 9 . Ring-opened 16 was drastically less active than 14; similarly, macrocycles ring expanded by one and three atoms, respectively (17 and 18), also lost binding affinity, confirming the critical role of the macrocycle for inhibition of Keap1. The activity of $\mathbf{1 4}$ in the ISA was confirmed in a direct SPR binding assay ${ }^{31}$ and by isothermal titration calorimetry (ITC), which provided almost identical affinities for Keap1 (Figure 2b,c). Macrocycle 14 displayed cellular activity as it induced $\mathrm{Nrf2}$ translocation into the nucleus by inhibiting binding to Keap1 (Figure 2d). Moreover, 14 has high aqueous solubility, low-to-moderate permeability across Caco- 2 cell monolayers, and a medium in vitro microsomal clearance. It therefore constitutes a promising lead compound for further optimization into an uncharged, nonacidic Keap 1 advanced lead.

Syntheses of Compounds 6-18. Compounds 6-18 were synthesized in five to seven steps from commercially available starting materials, using a modified version of the route reported for cyclothialidine (Scheme 1). ${ }^{29}$ The synthesis of macrocycles 6-9 (Scheme 1A) was initiated with the protection of 2(bromomethyl)benzoic acid as a 2,2,2-trichloroethyl (Tce) ester to give 19. Benzyl bromide 19 was used to alkylate the thiol of $\mathrm{L}-$ and D-cysteine methyl ester, respectively, providing 20a and 20b, which were then coupled with $\mathrm{L}$ - and D-Boc-serine affording dipeptides $\mathbf{2 1 a - d}$. The Tce protecting group was cleaved using zinc powder to obtain acids $22 \mathrm{a}-\mathbf{d}$, which were subjected to an intramolecular Mitsunobu reaction to obtain macrocycles 23ad. To our delight, the macrocyclization conditions proved robust, providing satisfactory yields for all four diastereoisomers 23a-d. Removal of the Boc group using acidic conditions, followed by coupling of the resulting free amine with Ac-L-Pro$\mathrm{OH}$, provided compounds 6-9.

Macrocycle 23d, the key intermediate in the synthetic sequence, was used to access compounds 10-15. After cleavage of its Boc group, coupling with Ac-D-Pro-OH or Ac-L-Hyp-OH provided compounds $\mathbf{1 0}$ and 11, respectively (Scheme 1B). The methyl ester of compound $23 \mathrm{~d}$ was cleaved using $\mathrm{Me}_{3} \mathrm{SnOH}$, as the use of traditional alkaline metal hydroxides led to partial opening of the lactone ring. The resulting crude acid was directly coupled with four different amines to give amides 24-27 (Scheme 1C). In a similar fashion as for compounds 6-9, 


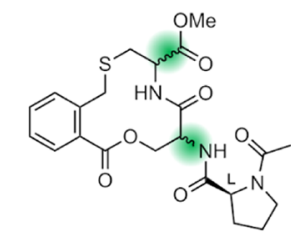

$6=\mathrm{L}-\mathrm{Cys}, \mathrm{L}-\mathrm{Ser} ; \mathrm{K}_{\mathrm{D}}>500 \mathrm{uM}$ 7 = D-Cys, L-Ser; $K_{D}>500 u M$ $8=$ L-Cys, D-Ser; $K_{0}>500 \mu M$ $9=\mathrm{D}$-Cys, D-Ser; $K_{D}=237 \pm 30 u M$

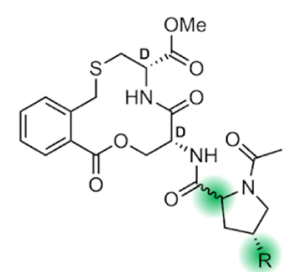

$10 \mathrm{D}-\mathrm{Pro}, \mathrm{R}=\mathrm{H} ; \mathrm{K}_{\mathrm{D}}>500 \mu \mathrm{M}$ $11 \mathrm{~L}-\mathrm{PrO}, \mathrm{R}=\mathrm{OH} ; \mathrm{K}_{\mathrm{D}}=270 \pm 99 \mathrm{uM}$

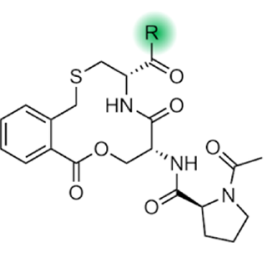

$12 \mathrm{R}=\mathrm{NH}_{2} ; \mathrm{K}_{\mathrm{D}}>500 \mu \mathrm{M}$ $13 \mathrm{R}=\mathrm{NHMe} ; \mathrm{K}_{\mathrm{D}}=209 \pm 95 u \mathrm{M}$ $14 \mathrm{R}=\mathrm{NMe}_{2} ; \mathrm{K}_{\mathrm{D}}=4.1 \pm 0.3 \mathrm{M}$ $15 \mathrm{R}=\mathrm{NEt}_{2} ; \mathrm{K}_{\mathrm{D}}>500 \mathrm{uM}$

Linear Control and Macrocycle Variations

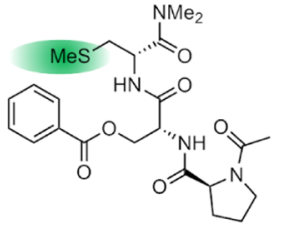

$16 ; K_{D}=406 \pm 55 \mu \mathrm{M}$

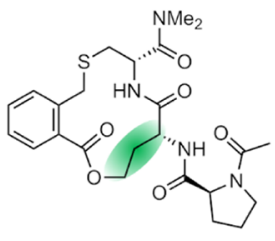

$17 ; \mathrm{K}_{\mathrm{D}}>500 \mu \mathrm{M}$

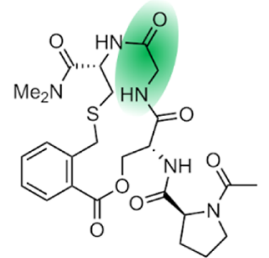

18: $K_{D}>500 u M$

b
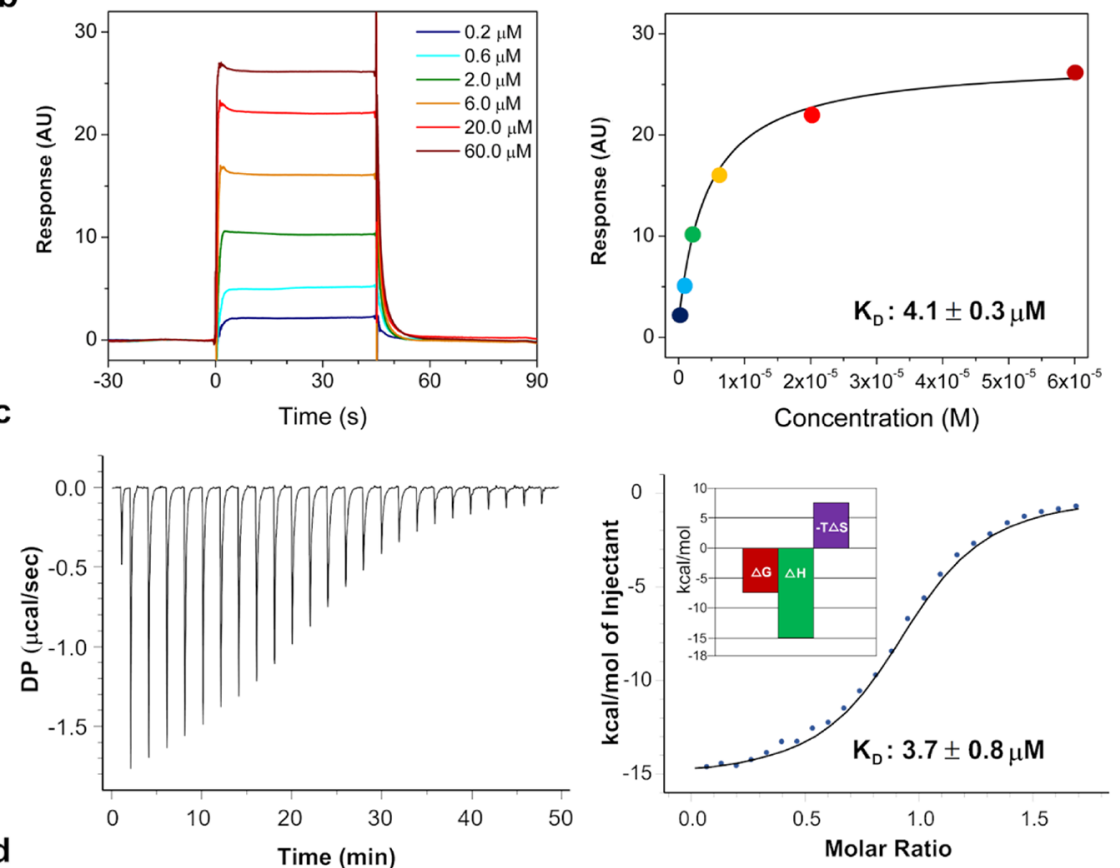

\begin{tabular}{c|c|c|c|c|c}
\hline $\begin{array}{c}\text { MW } \\
(\mathrm{Da})\end{array}$ & $\begin{array}{c}\text { TPSA } \\
(\AA)\end{array}$ & $\begin{array}{c}\text { Solubility } \\
(\mu \mathrm{M})\end{array}$ & $\begin{array}{c}\mathrm{P}_{\text {app }} \mathbf{A B}+\mathbf{I n h} \\
\left(\times 10^{-6} \mathbf{c m} / \mathbf{s}\right)\end{array}$ & $\begin{array}{c}\mathbf{C l}_{\text {int }} \\
(\mu \mathrm{L} / \mathrm{min} / \mathrm{mg})\end{array}$ & $\begin{array}{c}\text { Nrf2 transI } \\
(\% \text { induction })\end{array}$ \\
\hline 491 & 125 & $805 \pm 106$ & $1.6 \pm 0.42$ & $36.5 \pm 2.5$ & 40 \\
\hline
\end{tabular}

Figure 2. Characterization of cyclothialidine analogues. (a) Synthesized analogues of the cyclothialidine core that were evaluated as inhibitors of binding of Keap1 to an immobilized peptide derived from Nrf2 by surface plasmon resonance using an inhibition in solution assay (ISA) format. Dissociation constants, reported as mean values \pm standard deviation, from three measurements on three distinct samples are given for each analogue. (b) Interaction kinetic analysis of a dilution series of macrocycle $\mathbf{1 4}$ in a direct binding assay using immobilized Keap1 (left). Determination of the dissociation constant $\left(K_{\mathrm{D}}\right)$ for $\mathbf{1 4}$ by fitting of the data to a two-parametric sigmoidal equation (right). The dissociation constant was obtained from three measurements on three distinct samples and is reported as the mean value \pm standard deviation. (c) Determination of $K_{\mathrm{D}}$ for the binding of $\mathbf{1 4}$ to Keap 1 by isothermal titration calorimetry. The raw heat signals from the exothermic binding reaction (left) have been integrated to yield a binding isotherm (right) from which the thermodynamic parameters were extracted (insert). The dissociation constant was obtained from three measurements on three distinct samples and is reported as the mean value \pm standard deviation. (d) Characterization of macrocycle 14 by calculated descriptors (MW and TPSA), solubility in phosphate-buffered saline at $25^{\circ} \mathrm{C}$ and $\mathrm{pH} 7.4$, efflux-inhibited permeability across a Caco-2 cell monolayer $\left(\mathrm{P}_{\text {app }} \mathrm{AB}+\right.$ inh), human microsomal metabolism $\left(\mathrm{Cl}_{\text {int }}\right)$, and induction of $\mathrm{Nrf} 2$ translocation into the nucleus $(\mathrm{Nrf2}$ transl) at $256 \mu \mathrm{M}$. The values for solubility, cell permeability, and human microsomal metabolism are mean values \pm standard deviation from three measurements on three distinct samples. The Nrf2 translocation into the nucleus is the mean from two measurements on two distinct samples. 


\section{Scheme 1. Syntheses of Compounds $6-18^{a}$}

A. Synthesis of compounds 6-9
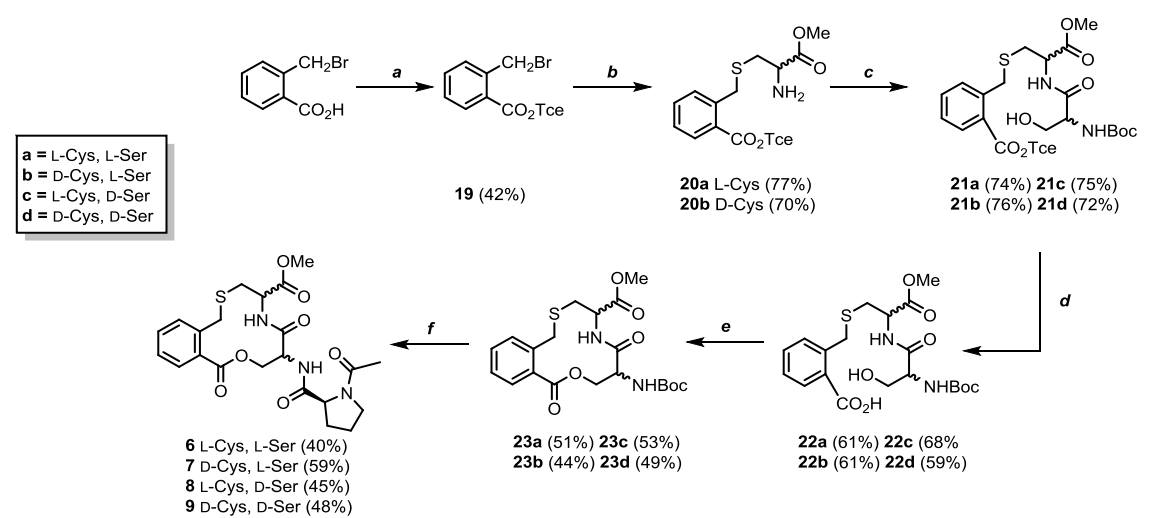

B. Synthesis of compounds 10 and 11

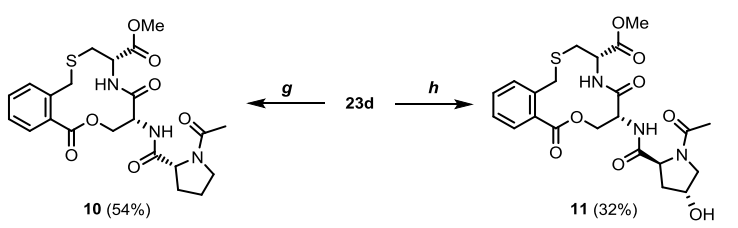

C. Synthesis of compounds 12-15

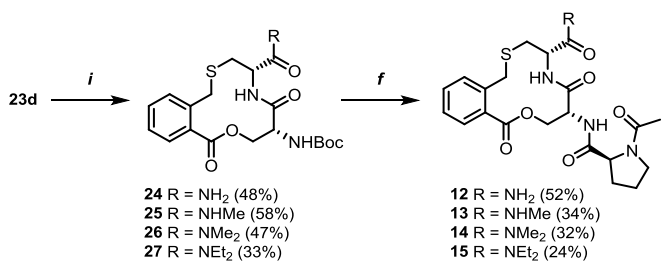

E. Synthesis of compound 17<smiles>COC(=O)c1ccccc1CBr</smiles>

$31(48 \%)$<smiles>CC(=O)N1CCCC1C(=O)N[C@@H](CCOC(=O)c1ccccc1CSCC(C)C)C(=O)N[C@@H](CSCc1ccccc1)C(=O)N(C)C</smiles>

$17(46 \%)$

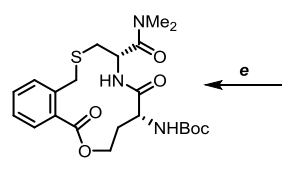

$34(21 \%)$

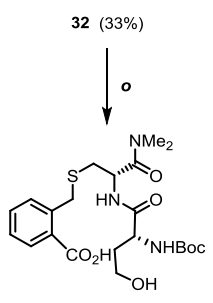

$33(64 \%)$

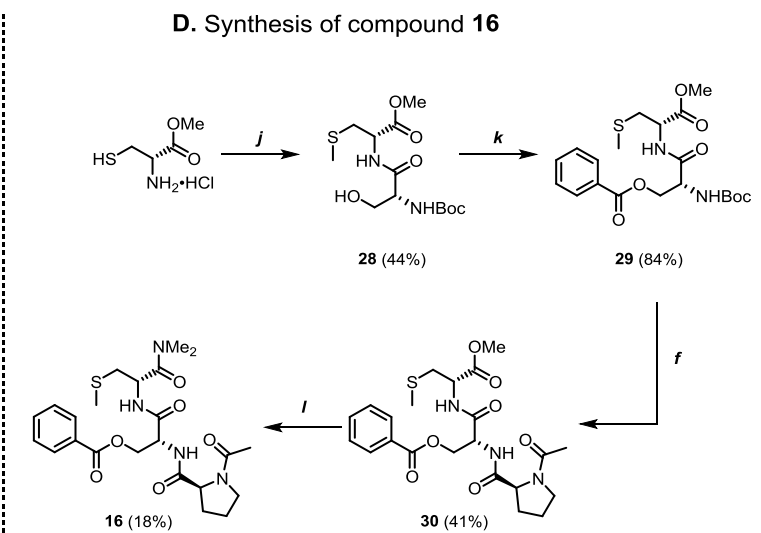

F. Synthesis of compound 18
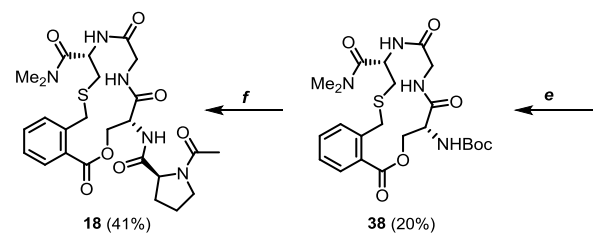

${ }^{a}$ Reagents and conditions: (a) 2,2,2-trichloroethanol, EDC $\cdot \mathrm{HCl}$, 4-dimethylaminopyridine (DMAP), dichloromethane (DCM), rt, 16 h; (b) H-(D/ L)-Cys-OMe $\cdot \mathrm{HCl}, \mathrm{Et}_{3} \mathrm{~N}, \mathrm{DMSO}, \mathrm{rt}, 2 \mathrm{~h}$; (c) Boc-(D/L)-Ser-OH, EDC $\cdot \mathrm{HCl}, \mathrm{MeCN}, \mathrm{rt}, 1$ h; (d) Zn dust, aq. $\mathrm{NH}_{4} \mathrm{OAc}$, tetrahydrofuran (THF), rt, 2 h; (e) $\mathrm{PPh}_{3}$, di-tert-butylazodicarboxylate, toluene:DMSO 95:5, rt, 4 h; (f) $\mathrm{HCl}$ in 1,4-dioxane, rt, $1 \mathrm{~h}$, then Ac-L-Pro-OH, EDC.HCl, N,Ndiisopropylethylamine (DIPEA), dimethyl sulfoxide (DMSO), rt, $2 \mathrm{~h} ;(\mathrm{g}) \mathrm{HCl}$ in 1,4-dioxane, $\mathrm{rt}, 1 \mathrm{~h}$, then Ac-D-Pro-OH, EDC·HCl, DIPEA, DMSO, rt, $2 \mathrm{~h}$; (h) $\mathrm{HCl}$ in 1,4-dioxane, rt, $1 \mathrm{~h}$, then Ac-L-Hyp-OH, EDC·HCl, DIPEA, DMSO, rt, $2 \mathrm{~h}$; (i) $\mathrm{Me}_{3} \mathrm{SnOH}, 1,2-\mathrm{DCE}, 83^{\circ} \mathrm{C}, 45 \mathrm{~min}$, then $\mathrm{R}_{2} \mathrm{NH}, \mathrm{EDC} \cdot \mathrm{HCl}, \mathrm{HOBt} \cdot \mathrm{H}_{2} \mathrm{O}, \mathrm{N}, \mathrm{N}$-dimethylformamide (DMF), rt, $2 \mathrm{~h}$; (j) MeI, DIPEA, DMSO, rt, $2 \mathrm{~h}$, then Boc-D-Ser-OH, EDC $\cdot \mathrm{HCl}$, $\mathrm{MeCN}, \mathrm{rt}, 1 \mathrm{~h}$; (k) BzCl, DMAP, Et ${ }_{3} \mathrm{~N}, \mathrm{DCM}, 0{ }^{\circ} \mathrm{C}$ to rt, $1 \mathrm{~h}$; (l) $\mathrm{Me}_{3} \mathrm{SnOH}, 1,2-\mathrm{DCE}, 83{ }^{\circ} \mathrm{C}, 45 \mathrm{~min}$, then $\mathrm{Me}{ }_{2} \mathrm{~N} \cdot \mathrm{HCl}, \mathrm{EDC} \cdot \mathrm{HCl}, \mathrm{HOBt} \cdot x \mathrm{H}_{2} \mathrm{O}$, DIPEA, DMF, rt, $2 \mathrm{~h}$; (m) Boc-D-Cys-OH, Et ${ }_{3} \mathrm{~N}$, THF:DMF 4:1, rt, $16 \mathrm{~h}$, then $\mathrm{Me}_{2} \mathrm{~N} \cdot \mathrm{HCl}, \mathrm{EDC} \cdot \mathrm{HCl}, \mathrm{HOBt} \cdot x \mathrm{H}_{2} \mathrm{O}, \mathrm{DIPEA}, \mathrm{DMF}, \mathrm{rt}, 2 \mathrm{~h}$; (n) $\mathrm{HCl}$ in 1,4-dioxane, rt, $1 \mathrm{~h}$, then Boc-D-Homoser-OH, EDC $\cdot \mathrm{HCl}$, DIPEA, $\mathrm{MeCN}, \mathrm{rt}, 1 \mathrm{~h}$; (o) aq. $\mathrm{LiOH}, \mathrm{MeOH}, 35^{\circ} \mathrm{C}, 16 \mathrm{~h}$; (p) $\mathrm{HCl}$ in $1,4-$ dioxane, $\mathrm{rt}, 1 \mathrm{~h}$, then Boc-Gly-OH, EDC $\cdot \mathrm{HCl}, \mathrm{MeCN}$, rt, $1 \mathrm{~h}$. DMAP, 4-dimethylaminopyridine; EDC, $N$-(3-dimethylaminopropyl)- $N^{\prime}$ ethylcarbodiimide; HOBt, 1-hydroxybenzotriazole; Tce, 2,2,2-trichloroethyl. 
cleavage of the Boc group from 24-27 and coupling with Ac-LPro-OH provided compounds 12-15.

The synthesis of ring-opened compound 16 (Scheme 1D) started with the methylation of the sulfur of D-cysteine methyl ester, followed by coupling with Boc-D-Ser to provide dipeptide 28, the hydroxyl group of which was acylated with benzoyl chloride in the presence of DMAP leading to compound 29. After Boc removal and coupling with Ac-L-Pro-OH tripeptide 30 was obtained. Cleavage of the methyl ester followed by coupling of the resulting free acid with dimethylamine afforded the linear control 16.

The preparation of the ring-expanded macrocycles $\mathbf{1 7}$ and $\mathbf{1 8}$ (Scheme 1E,F) is based on the common intermediate 31, which was prepared by alkylation of the thiol moiety of Boc-D-Cys with commercially available 2-bromomethyl methyl benzoate, followed by coupling of the crude carboxylic acid with dimethylamine. The Boc protecting group was then cleaved, the liberated amine was coupled with Boc-D-homoserine to give compound 32, followed by saponification of the methyl ester, which gives compounds 33 . The same conditions employed for the Mitsunobu reaction with acids $23 \mathbf{a}-\mathbf{d}$ were successfully applied to form the 13-membered lactone of 34. Finally, Boc removal and coupling with Ac-L-Pro-OH provided macrocycle 17. For the preparation of compound $\mathbf{1 8}$ (Scheme 1F), the Boc group of $\mathbf{3 1}$ was cleaved followed by coupling with Boc-glycine to obtain dipeptide 35 . The newly introduced Boc group was cleaved and the liberated amine was coupled with Boc-D-serine to afford tripeptide 36. After cleavage of the methyl ester, the obtained acid 37 was subjected to a Mitsunobu reaction to form the 15-membered core of compound 38, followed by Boc cleavage and coupling with Ac-L-Pro-OH to give macrocycle 18.

Structure of Inhibitor 14 in Complex with Keap1. We determined the structure of the complex of macrocycle 14 and Keap1 at $2.4 \AA$ resolution to understand how uncharged 14 binds in the charged and polar binding site of Keap 1 . The crystal structure confirms that $\mathbf{1 4}$ binds to the same polar binding site in Keap1 as Nrf2 (Figure 3a, Table S5, and Figure S8). Inspection of the structure shows that the phenylene group and parts of the macrocycle are wedged between R415 and A556 in a fairly hydrophobic pocket that reaches toward the Kelch channel, confirming that $\mathbf{1 4}$ is bound similarly to the docked poses of analogue 9 (Figure S9). The complex is stabilized only by a few polar interactions in addition to the cation $-\pi$ interaction between R415 and 14. The carbonyl group of Ser in 14 forms a hydrogen bond to the side chain of S602 in Keap1, similar to that of the main-chain carbonyl group of T 80 in Nrf2. ${ }^{32}$ In addition, a chloride ion bridges the $\mathrm{NH}$ of Ser in $\mathbf{1 4}$ with three residues in Keap 1, in a similar manner as in the complex with a highly potent inhibitor identified by fragment-based drug discovery. ${ }^{33}$ As physiological salt conditions were used in the SPR and ITC assays, as well as in the cellular Nrf2 translocation assay, we believe that formation of this complex also occurs in biologically relevant environments. The ability of macrocycle $\mathbf{1 4}$ to adopt a compact and well-defined conformation in the binding site explains why the ring-opened and ring-expanded analogues 1618 have a significantly lower affinity for Keap1. The solvent exposure of part of Pro and its $N$-acetyl group is in line with the fact that replacement with hydroxyproline (compound 11) does not affect the inhibitory potency.

The C-terminal dimethylamide of $\mathbf{1 4}$ is well-defined by the electron density of the X-ray structure and stacks against Y572, but inspection of the complex does not explain the large increase in potency as compared to ester 9 and amides 12, 13, and 15 .

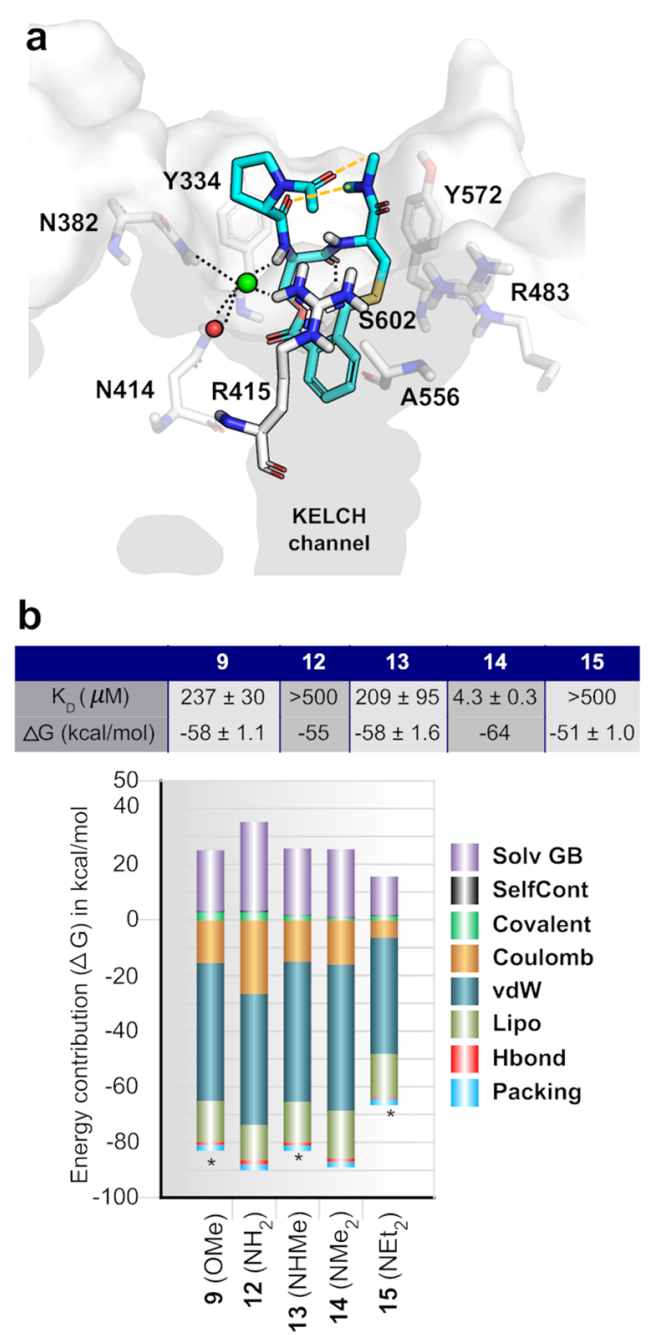

Figure 3. Characterization of the Keap1-14 complex. (a) Crystal structure of the complex between macrocyclic dimethylamide 14 and Keap1 determined at $2.4 \AA$ resolution (PDB ID: 6Z6A). Residues in Keap1 that make key contacts with $\mathbf{1 4}$ are shown in black text. The chloride ion that links R415, N414, and N382 to the NH of the serine moiety of macrocycle $\mathbf{1 4}$ is shown as a green sphere. An active site water that mediates formation of the protein-ligand complex is shown as a red sphere. The Kelch channel (dark gray) projects down from the phenylene group of 14. The nonclassical intramolecular hydrogen bonds that stabilize the bound structure of $\mathbf{1 4}$ are indicated with dashed orange lines. (b) Comparison of dissociation constants $\left(K_{\mathrm{D}}\right)$ with ligand binding affinities $(\Delta G)$ calculated with Prime MM-GBSA ${ }^{34}$ for the binding of $9,12,13,14$, and 15 to Keap 1 (top). Energy components that contribute to the binding affinities of $9,12,13,14$, and 15 in their complexes with Keap1 calculated with Prime MM-GBSA (bottom). ${ }^{34}$ Compounds 9, 13, and 15, which are marked with asterisks, may bind to Keap1 with their $\mathrm{OMe}, \mathrm{NHMe}$, and $\mathrm{NEt}_{2}$ groups in different conformations, e.g., the cis- and trans-orientations about the Cterminal amide bond of 13. Mean values for the energy components and standard deviations are shown here; values for each conformation are found in Tables S6-S7. Energy components: Solv GB, generalized Born electrostatic solvation energy; SelfCont, self-contact correction; Covalent, covalent binding energy; Coulomb, Coulomb energy; vdW, van der Waals energy; Lipo, lipophilic energy; Hbond, hydrogenbonding correction; Packing, $\pi-\pi$ packing correction.

Ligand binding affinity calculations using Prime MM-GBSA, ${ }^{34}$ which is frequently used to estimate the free energy of proteinligand complexes, ${ }^{35}$ provided insights both into the underlying reasons for the potency differences and into what forces stabilize 
the complex between 14 and Keap1 (Figure 3b, Tables S6-S7, Figure S10). In agreement with the experimental dissociation constants, macrocycle 14 was predicted to have the highest affinity for Keap1, with 9 and 13 being predicted as intermediate and 12 and 15 as weak. Nonpolar van der Waals and lipophilic interactions were found to be the main contributors to the binding affinity of $\mathbf{1 4}$ and to that it had a higher affinity than inhibitors 9 and 13. According to the calculations, the complex of the inactive $\mathbf{1 2}$ is stabilized by stronger polar (Coulomb) interactions, but this is offset by a larger desolvation penalty, while inactive $\mathbf{1 5}$ forms the weakest polar (Coulomb) and van der Waals interactions among the five compounds investigated. Interestingly, the distances between the two $N$-methyl groups of 14 and the carbonyl groups of Pro and the $\mathrm{N}$-acetyl group show that two intramolecular nonclassical hydrogen bonds ${ }^{36}$ are formed between these residues (Figure 3a), an observation that was supported by quantum mechanical calculations (see Supporting Information, Computational Procedure 2). Nonclassical hydrogen bonds are weaker than hydrogen bonds, ${ }^{37}$ but the two intramolecular bonds are likely to provide additional conformational restriction and stabilization of the Keap1-bound conformation of 14.

Comparison of 14 to Reported Keap1 Inhibitors. We assembled two sets of inhibitors of the Keap1-Nrf2 PPI to generate an overview of the diversity of the Keap1 inhibitor space and to investigate whether macrocycle 14 occupies a unique position in this space. The first set, termed "PubChem", was obtained from the 528 unique compounds reported as active in the PubChem Bioassay database after testing of $>337000$ compounds. ${ }^{38}$ Removal of compounds that did not pass a pan assay interference compounds (PAINS) filter ${ }^{39}$ from the actives gave the PubChem set consisting of 375 inhibitors (Table S8). A different set of "validated" inhibitors was assembled from two sources. First, nine compounds reported ${ }^{20}$ to show reproducible activity in a triad of orthogonal assays after resynthesis were included. Then, compounds bound in the binding site of Keap 1 for Nrf2 were retrieved from the Protein Data Bank (PDB). Peptides were excluded from this set, providing a "validated" set of 35 inhibitors, including six fragments that had a MW < $205 \mathrm{Da}$ (Table S9).

The Tanimoto coefficient, ${ }^{40}$ used to describe structural diversity, was calculated from seven fingerprints and revealed that $\mathbf{1 4}$ had a low similarity to the compounds in both the PubChem and the validated set (Figure $4 a$ and S11). The diversity of the Keap1 inhibitor space was investigated by networklike similarity graphs derived from substructure fragment fingerprints ${ }^{41}$ calculated for 14, the combined validated and PubChem sets, and the validated set alone. The graph for the combined set, just as the one for the validated set, confirmed that macrocycle 14 occupies a unique position in chemical space with no structurally similar neighbors (cf. color and size of spheres and the background, in Figure $4 b, c)$. The overview of the combined set also revealed the Keap 1 inhibitor space to be fairly diverse and that most inhibitors have few structural neighbors, with the exception of a large cluster located in the left center of the graph. This cluster contains a series of phthalimides represented by the validated inhibitor ${ }^{20} \mathrm{~V} 1$ (cf. structure in Figure $4 \mathrm{c}$ and Table S9). 1,4-Diaminonaphtalene V3, cyclic sulfonamide V7, and diazole V8 (Figure 4c) are the most potent reported Keap1 inhibitors and were obtained after medicinal chemistry optimization. In contrast to 14, they all contain carboxylic acids. In summary, both Tanimoto coefficients and the networklike similarity graphs highlight the potential of using
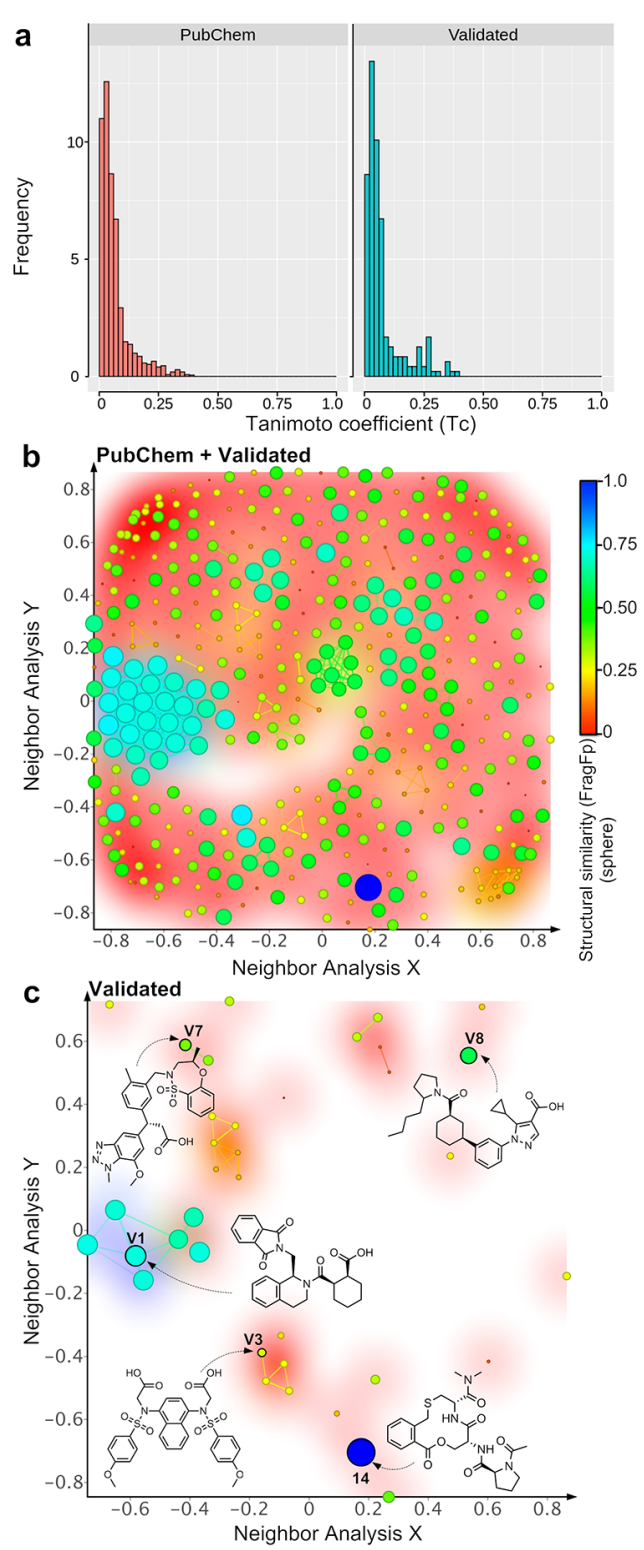

Figure 4. Structural similarity of inhibitor $\mathbf{1 4}$ to reported inhibitors of the Keap1-Nrf2 PPI. Comparisons have been made to a set of inhibitors retrieved from PubChem $(n=375)$ and to a validated set $(n=35)$. (a) Histograms of Tanimoto coefficients ${ }^{40}$ (Tc), calculated from seven structural fingerprints, comparing $\mathbf{1 4}$ to the PubChem and validated inhibitor sets. Inhibitors that have large structural differences as compared to 14 have $T c$ values close to 0 . (b, c) Network-like similarity graphs illustrating the structural diversity of the Keap1 inhibitor landscape and structural similarities, using 14 as the reference. The graphs were obtained after calculation of FragFp substructure fragment fingerprints ${ }^{41}$ for the compounds in the combined PubChem + validated set (panel $b$ ) and for the validated set only (panel $c$ ). Compounds that are similar to $\mathbf{1 4}$ are indicated by spheres that are similar in size and color to $\mathbf{1 4}$ (blue sphere). Connecting lines indicate clusters of inhibitors that have similar structures, and the number of similar neighbors is also highlighted with the background color (red, low number; blue, high number). Structures of four inhibitors from the validated set are shown together with compound $\mathbf{1 4}$ in panel $\mathrm{c}$.

natural-product-derived cores as a source of structurally unique Keap1 inhibitors, belonging to a chemical space very different from that of previously reported inhibitors. 


\section{DISCUSSION AND CONCLUSIONS}

Lead generation is very challenging for targets that have difficultto-drug binding sites, ${ }^{2-4}$ such as protein-protein interactions (PPIs). ${ }^{42}$ High-throughput screening (HTS) has often failed to identify useful hits for such targets as screening collections do not provide sufficient coverage of relevant chemical space due to their limited size and history of assembly. ${ }^{42}$ Fragment-based lead discovery allows a more comprehensive search of chemical space and has shown success in some cases where HTS has failed. ${ }^{13}$ Structure-based docking constitutes an alternative that may be particularly appealing for difficult-to-drug targets, as platforms for docking of ultralarge libraries are now being developed. ${ }^{43,44}$

Difficult-to-drug targets that have flat and featureless binding sites are often modulated by macrocyclic drugs, many of which originate from natural products. ${ }^{2,8}$ We therefore mined the macrocycles in the Dictionary of Natural Products to facilitate the identification of novel chemical matter for modulation of difficult-to-drug targets. This provided a smaller set of 41 leadlike macrocyclic cores and a larger set of 217 cores with more complex structures (Supporting Excel Sheet). Docking of the smaller set of cores into the positively charged and polar binding site of Keap 1 led to the discovery of the $4 \mu \mathrm{M}$ inhibitor 14, which originates from the core of cyclothialidine (1), after synthesis of only 13 compounds. Macrocycle 14 constitutes an uncharged inhibitor of Keap1, indicating that macrocycles may be used to discover promising starting points for difficult-to-drug targets that have polar binding sites, in addition to targets with flat and featureless sites. In contrast to $\mathbf{1 4}$, the majority of the reported Keap1 inhibitors contain acidic groups, which may be detrimental to cell permeability and oral absorption, ${ }^{21}$ and are expected to prevent CNS permeability. ${ }^{19}$ Compound $\mathbf{1 4}$ has druglike properties and shows cellular potency in an $\mathrm{Nrf} 2$ translocation assay, making it suitable for further lead optimization. The structure of its complex with Keap1 illustrates how cation $-\pi$ interactions may allow an uncharged ligand to bind in a charged binding site and provides a first indication of how an uncharged inhibitor can be tailored to fit the charged binding site.

It is interesting to contrast our results to those obtained in a virtual screen of 1.3 billion compounds from Enamine's REAL space library and the ZINC library, ${ }^{44}$ as well as with fragmentbased lead generation. ${ }^{33,45}$ The ultralarge screen identified Keap1 inhibitors with $100 \mathrm{nM}$ potencies as determined by surface plasmon resonance, ${ }^{44}$ but which contain potentially reactive or toxic groups. In addition, the structures were similar to previously reported inhibitors and appeared to provide limited opportunities for optimization into drug candidates. Obviously, screening of large libraries provides immense opportunities for drug discovery, but our results reiterate the importance of the structural diversity and quality of the libraries, just as for regular HTS compound collections. One of the most advanced Keap1 inhibitors was obtained by merging information from three fragments that bound in different subsites of the Keap1 binding site. ${ }^{33,45}$ While highly potent in in vitro $\left(K_{\mathrm{D}} 1.3\right.$ $\mathrm{nM})$ and active in cellular and in vivo models, the oral bioavailability in rats was low (7\%), potentially because of the presence of a carboxylic acid moiety originating from one of the fragments.

Recently, natural products have also been utilized as a source of three-dimensional (3D) fragments ${ }^{12}$ and as inspiration for design of compound collections prepared by diversity-oriented synthesis (DOS). ${ }^{46}$ In addition, pseudo-natural products have been designed by combination and fusion of natural-productderived fragments. ${ }^{47}$ However, natural products and their derivatives often have complex structures. We demonstrated how two rounds of docking of our smaller set of lead-like cores first identified the cyclothialidine core and then tailored it to its target Keap1 via docking of 6-9 while simultaneously reducing the structural complexity. In this manner, one of the major shortcomings of natural products, i.e., that they are often prepared via long synthetic routes, was mitigated. Similarly, the larger set of 217 structurally more complex macrocyclic cores may provide inspiration for design of collections having simplified structures that can be prepared through shorter routes than the original natural products. In conclusion, the cores reported herein provide another example of how nature's privileged structures and their diversity can be capitalized on as a rich source of quality leads for drug discovery. ${ }^{11}$

\section{EXPERIMENTAL SECTION}

Chemistry. General Methods. All reagents were purchased from Sigma-Aldrich, Fluorochem, and VWR International. DCM, DMSO, hexane, DMF, and acetonitrile were purchased from VWR International, while 1,2-DCE, toluene, and THF were purchased from SigmaAldrich. All nonaqueous reactions were performed in oven-dried glassware under an argon atmosphere. The Büchi rotary evaporator R114 was used to remove solvents in vacuo. Reactions were generally monitored by liquid chromatography-mass spectrometry (LC-MS) with an Agilent 1100 series high-performance liquid chromatography (HPLC) with a C18 Atlantis T3 column $(3.0 \mathrm{~mm} \times 50 \mathrm{~mm}, 5 \mu \mathrm{m})$ using acetonitrile-water (flow rate $0.75 \mathrm{~mL} / \mathrm{min}$ over $6 \mathrm{~min}$ ) as the mobile phase and a Waters micromass ZQ (model code: MM1) mass spectrometer with the electrospray ionization mode as the detector. Alternatively, TLC silica gel 60 F254 plates from VWR International were used and visualization was done using UV light $(254 \mathrm{~nm})$ or by staining with a $\mathrm{KMnO}_{4}$ solution $(2 \% \mathrm{~m} / \mathrm{v}$ in water). Silica gel (43-63 $\mu \mathrm{m}$, VWR international) was used for purification of compounds with flash column chromatography. Preparative reversed-phase HPLC was performed on a Kromasil C8 column $(250 \mathrm{~mm} \times 21.2 \mathrm{~mm}, 5 \mu \mathrm{m})$ on a Gilson HPLC equipped with a Gilson 322 pump, a UV-visible-156 detector, and a 202 collector using acetonitrile-water gradients as eluents with a flow rate of $15 \mathrm{~mL} / \mathrm{min}$ and detection at 214 or $254 \mathrm{~nm}$. ${ }^{1} \mathrm{H},{ }^{13} \mathrm{C}$, COSY, HSQC, and HMBC NMR spectra for synthesized compounds were recorded at $298 \mathrm{~K}$ on an Agilent Technologies 400 MR spectrometer at 400 or $100 \mathrm{MHz}$ or on an OXFORD AS500 spectrometer at 500 or $126 \mathrm{MHz}$ or on a Bruker Avance III spectrometer at $600 \mathrm{MHz}$ or at $151 \mathrm{MHz}$. The residual peak of the respective solvent was used as the internal standard $\left[\mathrm{CDCl}_{3}\left(\mathrm{CHCl}_{3}\right.\right.$ $\left.\delta \mathrm{H} 7.26 \mathrm{ppm}, \mathrm{CDCl}_{3} \delta \mathrm{C} 77.0 \mathrm{ppm}\right)$, DMSO- $d_{6}\left(\mathrm{CD}_{2} \mathrm{HSOCD}_{3} \delta \mathrm{H} 2.50\right.$ ppm, $\left.\mathrm{CD}_{3} \mathrm{SOCD}_{3} \delta \mathrm{C} 39.5 \mathrm{ppm}\right), \mathrm{CD}_{3} \mathrm{OD}\left(\mathrm{CD}_{2} \mathrm{HOD} \delta \mathrm{H} 3.31 \mathrm{ppm}\right.$, $\left.\mathrm{CD}_{3} \mathrm{OD} \delta \mathrm{C} 49.0 \mathrm{ppm}\right), \mathrm{CD}_{3} \mathrm{CN}\left(\mathrm{CD}_{2} \mathrm{HCN} \delta \mathrm{H} 1.94 \mathrm{ppm}, \mathrm{CD}_{3} \mathrm{CN} \delta \mathrm{C}\right.$ 1.3 and $118.3 \mathrm{ppm})$, acetone- $d_{6}\left(\mathrm{CD}_{2} \mathrm{HCOCD}_{3} \delta \mathrm{H} 2.05 \mathrm{ppm}\right.$, $\mathrm{CD}_{3} \mathrm{COCD}_{3} \delta \mathrm{C} 29.8$ and $\left.\left.206.3 \mathrm{ppm}\right)\right]$. HRMS for all new compounds were recorded in the electrospray ionization (ESI) mode on an S3 LCT Premier connected to a Waters acquity UPLC I-class with acetonitrilewater used as the mobile phase $(1: 1$, with a flow rate of $0.25 \mathrm{~mL} / \mathrm{min})$. The purity of compounds $\mathbf{6 - 1 8}$ is $\geq 95 \%$ as determined using a Waters LCT Premiere mass spectrometer coupled to a Waters Acquity UPLC. The Waters Acquity UPLC was equipped with either a $\mathrm{BEH} \mathrm{C18}$ column $\left(1.7 \mu \mathrm{m}, 2.1 \mathrm{~mm} \times 50 \mathrm{~mm}\right.$, at $45^{\circ} \mathrm{C}$ using a gradient from 5 to $90 \%$ acetonitrile modified with $40 \mathrm{mM}$ ammonia and $5 \mathrm{mM} \mathrm{H}_{2} \mathrm{CO}_{3}, \mathrm{pH}$ 10 within 2.5 or $3 \mathrm{~min}$, detection at $210 \mathrm{~nm})$ or a CSH C18 column (1.7 $\mu \mathrm{m}, 2.1 \mathrm{~mm} \times 50 \mathrm{~mm}$ at $45{ }^{\circ} \mathrm{C}$ using a gradient from 5 to $90 \%$ acetonitrile modified with $10 \mathrm{mM}$ formic acid and $1 \mathrm{mM}$ ammonium formate, $\mathrm{pH} 3$, within 2.5 or $3 \mathrm{~min}$, detection at $230 \mathrm{~nm}$ ).

2,2,2-Trichloroethyl 2-(bromomethyl)benzoate (19). 2-Methylbenzoic acid ( $8.00 \mathrm{~g}, 37.2 \mathrm{mmol}, 1.0$ equiv) was dissolved in DCM (30 $\mathrm{mL})$. EDC $\cdot \mathrm{HCl}(10.7 \mathrm{~g}, 55.8 \mathrm{mmol}, 1.5$ equiv), DMAP (454 mg, 3.72 mmol, 0.1 equiv), and 2,2,2-trichloroethanol ( $4.65 \mathrm{~mL}, 48.4 \mathrm{mmol}, 1.3$ equiv) were then added, and the mixture was stirred at $\mathrm{rt}$ for $16 \mathrm{~h}$. The 
mixture was diluted with DCM $(150 \mathrm{~mL})$ and washed with $1 \mathrm{M}$ aqueous $\mathrm{HCl}$ solution $(2 \times 100 \mathrm{~mL})$ and saturated aqueous $\mathrm{NaHCO}_{3}$ solution $(2 \times 100 \mathrm{~mL})$. The organic phase was dried over $\mathrm{MgSO}_{4}$, filtered, and then concentrated under reduced pressure. The crude mixture was purified by flash chromatography on a silica gel column using 2-10\% EtOAc in hexane as the eluent to give 19 (5.41 g, 15.6 $\mathrm{mmol}, 42 \%)$ as a colorless oil. The ${ }^{1} \mathrm{H}$ NMR spectrum is in accordance with the literature data. ${ }^{48}$

2,2,2-Trichloroethyl-(R)-2-(((2-amino-3-methoxy-3-oxopropyl)thio)methyl)benzoate (20a). 2,2,2-Trichloroethyl 2-(bromomethyl)benzoate 19 ( $3.46 \mathrm{~g}, 10.0 \mathrm{mmol}, 1.0$ equiv) was dissolved in DMSO (50 $\mathrm{mL})$, L-cysteine methyl ester hydrochloride $(2.06 \mathrm{~g}, 12.0 \mathrm{mmol}, 1.2$ equiv) and triethylamine $(3.10 \mathrm{~mL}, 22.0 \mathrm{mmol}, 2.2$ equiv $)$ were added, and the mixture was stirred for $2 \mathrm{~h}$ at rt. The mixture was diluted with EtOAc $(250 \mathrm{~mL})$ and washed with saturated aqueous $\mathrm{NaHCO}_{3}$ solution $(2 \times 100 \mathrm{~mL})$ and brine $(100 \mathrm{~mL})$. The organic phase was dried over $\mathrm{MgSO}_{4}$, filtered, and then removed under reduced pressure. The crude reaction mixture was purified by flash chromatography on a silica gel column using $0-10 \% \mathrm{MeOH}$ in DCM as the eluent to give 20a (3.09 g, $7.70 \mathrm{mmol}, 77 \%$ ) as a yellow oil. HRMS (ESI) $\mathrm{m} / z$ calcd for $\mathrm{C}_{14} \mathrm{H}_{17} \mathrm{Cl}_{3} \mathrm{NO}_{4} \mathrm{~S}[\mathrm{M}+\mathrm{H}]^{+}$399.9944, found 399.9953. ${ }^{1} \mathrm{H}$ NMR (400 $\left.\mathrm{MHz}, \mathrm{CDCl}_{3}\right) \delta 8.08(\mathrm{dd}, J=7.8,1.4 \mathrm{~Hz}, 1 \mathrm{H}), 7.52(\mathrm{td}, J=7.8,1.4 \mathrm{~Hz}$, $1 \mathrm{H}), 7.42-7.32(\mathrm{~m}, 2 \mathrm{H}), 4.97(\mathrm{~s}, 2 \mathrm{H}), 4.23(\mathrm{~d}, J=13.2 \mathrm{~Hz}, 1 \mathrm{H}), 4.16$ (d, $J=13.2 \mathrm{~Hz}, 1 \mathrm{H}), 3.72(\mathrm{~s}, 3 \mathrm{H}), 3.63(\mathrm{dd}, J=7.6,4.6 \mathrm{~Hz}, 1 \mathrm{H}), 2.87$ (dd, $J=13.6,4.6 \mathrm{~Hz}, 1 \mathrm{H}), 2.68(\mathrm{dd}, J=13.6,7.6 \mathrm{~Hz}, 1 \mathrm{H}), 1.79(\mathrm{br} \mathrm{s}$, $2 \mathrm{H}) .{ }^{13} \mathrm{C} \mathrm{NMR}\left(101 \mathrm{MHz}, \mathrm{CDCl}_{3}\right) \delta 174.3,165.2,141.0,132.8,131.8$, $131.3,127.7,127.5,95.0,74.6,54.2,52.2,36.9,34.8$.

2,2,2-Trichloroethyl 2-((4R,7S)-7-(Hydroxymethyl)-4-(methoxycarbonyl)-11,11-dimethyl-6,9-dioxo-10-oxa-2-thia-5,8diazadodecyl)benzoate (21a). Compound 20a (1.25 g, $3.13 \mathrm{mmol}$, 1.0 equiv) was dissolved in acetonitrile $(18 \mathrm{~mL})$, Boc-L-Ser-OH $(0.96 \mathrm{~g}$, $4.71 \mathrm{mmol}, 1.5$ equiv) and $\mathrm{EDC} \cdot \mathrm{HCl}(0.89 \mathrm{~g}, 4.71 \mathrm{mmol}, 1.5$ equiv) were added, and the reaction was stirred for $1 \mathrm{~h}$ at rt. EtOAc $(150 \mathrm{~mL})$ was then added, and the mixture was washed with $1 \mathrm{M}$ aqueous $\mathrm{HCl}$ solution $(100 \mathrm{~mL})$, saturated aqueous $\mathrm{NaHCO}_{3}$ solution $(100 \mathrm{~mL})$, and brine $(100 \mathrm{~mL})$. The organic phase was dried over $\mathrm{MgSO}_{4}$, filtered, and then concentrated under reduced pressure. The crude reaction mixture was purified by flash chromatography on a silica gel column using $0-5 \% \mathrm{MeOH}$ in DCM as the eluent to give 21a (1.35 g, 2.31 mmol, $74 \%$ ) as a colorless foam. HRMS (ESI) $\mathrm{m} / z$ calcd for $\mathrm{C}_{22} \mathrm{H}_{30} \mathrm{Cl}_{3} \mathrm{~N}_{2} \mathrm{O}_{8} \mathrm{~S}[\mathrm{M}+\mathrm{H}]^{+}$587.0788, found 578.0794. ${ }^{1} \mathrm{H}$ NMR $\left(400 \mathrm{MHz}, \mathrm{CDCl}_{3}\right) \delta 8.08(\mathrm{~d}, J=8.0 \mathrm{~Hz}, 1 \mathrm{H}), 7.50(\mathrm{t}, J=8.0 \mathrm{~Hz}, 1 \mathrm{H})$, $7.40-7.34(\mathrm{~m}, 3 \mathrm{H}), 5.62(\mathrm{~d}, J=7.4 \mathrm{~Hz}, 1 \mathrm{H}), 4.96(\mathrm{~s}, 2 \mathrm{H}), 4.85-4.75$ $(\mathrm{m}, 1 \mathrm{H}), 4.29-4.25(\mathrm{~m}, 1 \mathrm{H}), 4.23(\mathrm{~d}, J=12.8 \mathrm{~Hz}, 1 \mathrm{H}), 4.10(\mathrm{~d}, J=12.8$ $\mathrm{Hz}, 1 \mathrm{H}), 4.03(\mathrm{dd}, J=11.5,3.6 \mathrm{~Hz}, 1 \mathrm{H}), 3.71(\mathrm{~s}, 3 \mathrm{H}), 3.69-3.64(\mathrm{~m}$, $1 \mathrm{H}), 3.20(\mathrm{br} \mathrm{s}, 1 \mathrm{H}), 2.94(\mathrm{dd}, J=14.0,4.8 \mathrm{~Hz}, 1 \mathrm{H}), 2.86(\mathrm{dd}, J=14.0$, $6.3 \mathrm{~Hz}, 1 \mathrm{H}), 1.43(\mathrm{~s}, 9 \mathrm{H}) .{ }^{13} \mathrm{C} \mathrm{NMR}\left(101 \mathrm{MHz}, \mathrm{CDCl}_{3}\right) \delta 171.2,170.9$, 165.2, 155.8, 140.4, 133.0, 131.8, 131.4, 127.7, 127.6, 94.9, 80.9, 74.6, 63.1, 55.3, 52.8, 52.1, 34.8, 33.5, 28.3.

2-((4R,7S)-7-(Hydroxymethyl)-4-(methoxycarbonyl)-11,11-dimethyl-6,9-dioxo-10-oxa-2-thia-5,8-diazadodecyl)benzoic Acid (22a). Compound $21 \mathrm{a}$ (1.35 g, $2.31 \mathrm{mmol}, 1.0$ equiv) was dissolved in THF $(19 \mathrm{~mL})$ and aqueous $1 \mathrm{M} \mathrm{NH}_{4} \mathrm{OAc}(4 \mathrm{~mL})$. $\mathrm{Zn}$ dust $(1.51 \mathrm{~g}$, $23.1 \mathrm{mmol}, 10$ equiv) was added, and the reaction was stirred for $2 \mathrm{~h}$ at rt. The mixture was diluted with $\mathrm{MeOH}(250 \mathrm{~mL})$, filtered through a pad of Celite, and concentrated under reduced pressure. The crude reaction mixture was purified by flash chromatography on a silica gel column using $0-20 \% \mathrm{MeOH} / \mathrm{EtOAc}$ as the eluent to give $22 \mathrm{a}(642 \mathrm{mg}$, $1.41 \mathrm{mmol}, 61 \%)$ as a colorless powder. The ${ }^{1} \mathrm{H}$ NMR spectrum is in accordance with the literature data. ${ }^{29}$

Methyl (4R,7S)-7-((tert-Butoxycarbonyl)amino)-6,10-dioxo$1,3,4,5,6,7,8,10$-octahydrobenzo[j][1] oxa[8] thia[5]azacyclododecine-4-carboxylate (23a). Compound 22a (640 mg, $1.40 \mathrm{mmol}, 1.0$ equiv) was dissolved in toluene $(33 \mathrm{~mL})$ and DMSO (2 $\mathrm{mL})$. Triphenylphosphine $(550 \mathrm{mg}, 2.10 \mathrm{mmol}, 1.5$ equiv) and di-tertbutyl azodicarboxylate $(483 \mathrm{mg}, 2.10 \mathrm{mmol}, 1.5$ equiv) were then added, and the mixture was stirred for $4 \mathrm{~h}$ at $\mathrm{rt}$. After evaporation of the solvent, the crude product was purified by flash chromatography on a silica gel column using $40-60 \%$ EtOAc in hexane as the eluent to give 23a (313 mg, $0.71 \mathrm{mmol}, 51 \%$ ) as a colorless powder. The ${ }^{1} \mathrm{H}$ NMR spectrum was in accordance with the literature data. ${ }^{29}$

Methyl-(4R,7S)-7-((S)-1-acetylpyrrolidine-2-carboxamido)-6,10dioxo-1,3,4,5,6,7,8,10-octahydrobenzo[j][1]oxa[8]thia[5]azacyclododecine-4-carboxylate (6). Compound $6(100 \mathrm{mg}, 0.21$ mmol, 1.0 equiv) was dissolved in $4 \mathrm{M} \mathrm{HCl}$ in dioxane $(5 \mathrm{~mL})$ and stirred for $1 \mathrm{~h}$ at $\mathrm{rt}$. After evaporation of the solvent under reduced pressure, the resulting salt was dissolved in DMSO $(4 \mathrm{~mL})$; Ac-L-Pro$\mathrm{OH}$ ( $70 \mathrm{mg}, 0.42 \mathrm{mmol}, 2.0$ equiv), $\mathrm{EDC} \cdot \mathrm{HCl}(86 \mathrm{mg}, 0.42 \mathrm{mmol}, 2.0$ equiv), and DIPEA ( $39 \mu \mathrm{L}, 0.21 \mathrm{mmol}, 1.0$ equiv) were added, and the reaction was stirred for $2 \mathrm{~h}$ at rt. The crude product was purified by reverse-phase HPLC using a gradient from 30 to $75 \%$ acetonitrile in water to give 6 ( $43 \mathrm{mg}, 0.18 \mathrm{mmol}, 40 \%$ ) as a colorless powder. HRMS (ESI) $m / z$ calcd for $\mathrm{C}_{22} \mathrm{H}_{28} \mathrm{~N}_{3} \mathrm{O}_{7} \mathrm{~S}[\mathrm{M}+\mathrm{H}]^{+} 478.1648$, found 478.1652. ${ }^{1} \mathrm{H}$ NMR $\left(400 \mathrm{MHz}, \mathrm{CDCl}_{3}\right) \delta 7.73(\mathrm{dd}, J=7.8,1.4 \mathrm{~Hz}, 1 \mathrm{H})$, $7.68(\mathrm{~d}, J=8.8 \mathrm{~Hz}, 1 \mathrm{H}), 7.52-7.42(\mathrm{~m}, 2 \mathrm{H}), 7.37(\mathrm{dd}, J=7.6,1.4 \mathrm{~Hz}$, $1 \mathrm{H}), 7.32(\mathrm{td}, J=7.8,1.4 \mathrm{~Hz}, 1 \mathrm{H}), 5.00-4.86(\mathrm{~m}, 3 \mathrm{H}), 4.68-4.55(\mathrm{~m}$, $1 \mathrm{H}), 4.51(\mathrm{dd}, J=7.4,5.3 \mathrm{~Hz}, 1 \mathrm{H}), 4.02(\mathrm{~d}, J=10.3 \mathrm{~Hz}, 1 \mathrm{H}), 3.81(\mathrm{~d}, J$ $=10.3 \mathrm{~Hz}, 1 \mathrm{H}), 3.75(\mathrm{~s}, 3 \mathrm{H}), 3.73-3.66(\mathrm{~m}, 1 \mathrm{H}), 3.55-3.44(\mathrm{~m}, 1 \mathrm{H})$, $3.26(\mathrm{dd}, J=14.5,4.8 \mathrm{~Hz}, 1 \mathrm{H}), 2.96(\mathrm{dd}, J=14.5,9.7 \mathrm{~Hz}, 1 \mathrm{H}), 2.27-$ $2.16(\mathrm{~m}, 2 \mathrm{H}), 2.11-1.94(\mathrm{~m}, 2 \mathrm{H}), 2.03(\mathrm{~s}, 3 \mathrm{H}) .{ }^{13} \mathrm{C}$ NMR $(101 \mathrm{MHz}$, $\left.\mathrm{CDCl}_{3}\right) \delta 172.0,171.1,170.8,169.6,168.9,136.4,132.3,131.8,130.8$, $130.7,127.5,67.1,61.1,53.8,52.7,51.6,48.5,34.7,34.4,29.2,25.0$, 22.8 .

2,2,2-Trichloroethyl (S)-2-(((2-Amino-3-methoxy-3-oxopropyl)thio)methyl)benzoate (20b). Compound $20 \mathrm{~b}$ was prepared following the procedure described for the synthesis of compound 20a using 2,2,2trichloroethyl 2-(bromomethyl)benzoate $19(1.70 \mathrm{~g}, 4.91 \mathrm{mmol}, 1.0$ equiv), D-cysteine methyl ester hydrochloride ( $1.01 \mathrm{~g}, 5.89 \mathrm{mmol}, 1.2$ equiv), and triethylamine ( $1.53 \mathrm{~mL}, 10.8 \mathrm{mmol}, 2.2$ equiv). The crude reaction mixture was purified by flash chromatography on a silica gel column using $0-10 \% \mathrm{MeOH}$ in DCM as the eluent to give $20 \mathrm{~b}$ ( $1.37 \mathrm{~g}$, $3.43 \mathrm{mmol}, 70 \%$ ) as a yellow oil. HRMS (ESI) $\mathrm{m} / z$ calcd for $\mathrm{C}_{14} \mathrm{H}_{16} \mathrm{Cl}_{3} \mathrm{NNaO}_{4} \mathrm{~S}[\mathrm{M}+\mathrm{Na}]^{+}$421.9763, found 421.9770. ${ }^{1} \mathrm{H} \mathrm{NMR}$ $\left(400 \mathrm{MHz}, \mathrm{CDCl}_{3}\right) \delta 8.07(\mathrm{dd}, J=7.7,1.5 \mathrm{~Hz}, 1 \mathrm{H}), 7.52-7.47(\mathrm{~m}$, $1 \mathrm{H}), 7.43-7.28(\mathrm{~m}, 2 \mathrm{H}), 4.95(\mathrm{~s}, 2 \mathrm{H}), 4.21(\mathrm{~d}, J=13.2 \mathrm{~Hz}, 1 \mathrm{H}), 4.14$ $(\mathrm{d}, J=13.2 \mathrm{~Hz}, 1 \mathrm{H}), 3.70(\mathrm{~s}, 3 \mathrm{H}), 3.60(\mathrm{dd}, J=7.6,4.6 \mathrm{~Hz}, 1 \mathrm{H}), 2.85$ (dd, $J=13.6,4.6 \mathrm{~Hz}, 1 \mathrm{H}), 2.66$ (dd, $J=13.6,7.6 \mathrm{~Hz}, 1 \mathrm{H}), 1.74(\mathrm{br} \mathrm{s}$,

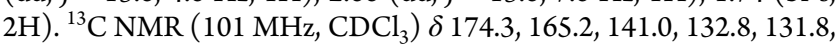
$131.3,127.7,127.5,95.0,74.6,54.1,52.2,36.9,34.8$.

2,2,2-Trichloroethyl 2-((4S,7S)-7-(Hydroxymethyl)-4-(methoxycarbonyl)-11,11-dimethyl-6,9-dioxo-10-oxa-2-thia-5,8diazadodecyl)benzoate (21b). Compound $21 \mathrm{~b}$ was prepared following the procedure described for the synthesis of compound 21a using $20 \mathrm{~b}$ ( $1.30 \mathrm{~g}, 3.25 \mathrm{mmol}, 1.0$ equiv), Boc-L-Ser-OH ( $990 \mathrm{mg}$, 4.87 mmol, 1.5 equiv), and $\mathrm{EDC} \cdot \mathrm{HCl}$ ( $932 \mathrm{mg}, 4.88 \mathrm{mmol}, 1.5$ equiv). The crude reaction mixture was purified by flash chromatography on a silica gel column using $0-5 \% \mathrm{MeOH}$ in DCM as the eluent to give $21 \mathbf{b}$ (1.45 g, $2.47 \mathrm{mmol}, 76 \%$ ) as a colorless foam. HRMS (ESI) $\mathrm{m} / z$ calcd for $\mathrm{C}_{22} \mathrm{H}_{30} \mathrm{Cl}_{3} \mathrm{~N}_{2} \mathrm{O}_{8} \mathrm{~S}[\mathrm{M}+\mathrm{H}]^{+} 587.0788$, found 587.0773. ${ }^{1} \mathrm{H}$ NMR (400 $\left.\mathrm{MHz}, \mathrm{CDCl}_{3}\right) \delta 8.08(\mathrm{dd}, J=8.0,1.4 \mathrm{~Hz}, 1 \mathrm{H}), 7.50(\mathrm{td}, J=8.0,1.4 \mathrm{~Hz}$, $1 \mathrm{H}), 7.40-7.34(\mathrm{~m}, 2 \mathrm{H}), 7.36-7.31(\mathrm{~m}, 1 \mathrm{H}), 5.62(\mathrm{~d}, J=7.4 \mathrm{~Hz}, 1 \mathrm{H})$, $4.99(\mathrm{~d}, J=12.0 \mathrm{~Hz}, 1 \mathrm{H}), 4.96(\mathrm{~d}, J=12.0 \mathrm{~Hz}, 1 \mathrm{H}), 4.85-4.75(\mathrm{~m}, 1 \mathrm{H})$, $4.27-4.18(\mathrm{~m}, 2 \mathrm{H}), 4.10(\mathrm{~d}, J=12.8 \mathrm{~Hz}, 1 \mathrm{H}), 4.09-4.04(\mathrm{~m}, 1 \mathrm{H}), 3.71$ $(\mathrm{s}, 3 \mathrm{H}), 3.68(\mathrm{dd}, J=11.6,4.9 \mathrm{~Hz}, 1 \mathrm{H}), 2.97-2.84(\mathrm{~m}, 2 \mathrm{H}), 2.38(\mathrm{br} \mathrm{s}$, $1 \mathrm{H}), 1.43(\mathrm{~s}, 9 \mathrm{H}) .{ }^{13} \mathrm{C} \mathrm{NMR}\left(101 \mathrm{MHz}, \mathrm{CDCl}_{3}\right) \delta 171.5,171.0,165.2$, 156.0, 140.4, 133.0, 131.8, 131.4, 127.7, 127.6, 94.9, 80.5, 74.6, 63.1, $55.3,52.8,52.0,34.8,33.4,28.3$.

2-((4S,7S)-7-(Hydroxymethyl)-4-(methoxycarbonyl)-11,11-dimethyl-6,9-dioxo-10-oxa-2-thia-5,8-diazadodecyl)benzoic Acid (22b). Compound $22 \mathrm{~b}$ was prepared following the procedure described for the synthesis of compound 22a using $21 \mathbf{b}(1.20 \mathrm{~g}, 2.04 \mathrm{mmol}, 1.0$ equiv) and $\mathrm{Zn}$ dust ( $1.33 \mathrm{~g}, 20.4 \mathrm{mmol}, 10$ equiv). The crude reaction mixture was purified by flash chromatography on a silica gel column using $0-20 \% \mathrm{MeOH}$ in EtOAc as the eluent to give 22b (567 mg, 1.24 mmol, $61 \%$ ) as a colorless powder. HRMS (ESI) $\mathrm{m} / z$ calcd for $\mathrm{C}_{20} \mathrm{H}_{29} \mathrm{~N}_{2} \mathrm{O}_{8} \mathrm{~S}[\mathrm{M}+\mathrm{H}]^{+}$457.1645, found 457.1637. ${ }^{1} \mathrm{H}$ NMR (400 MHz, DMSO-d $)_{6} \delta 12.93(\mathrm{br} \mathrm{s}, 1 \mathrm{H}), 8.27(\mathrm{~d}, J=7.8 \mathrm{~Hz}, 1 \mathrm{H}), 7.82(\mathrm{dd}$, $J=8.0,1.7 \mathrm{~Hz}, 1 \mathrm{H}), 7.46(\mathrm{td}, J=7.6,1.7 \mathrm{~Hz}, 1 \mathrm{H}), 7.41-7.29(\mathrm{~m}, 2 \mathrm{H})$, $6.63(\mathrm{~d}, J=8.1 \mathrm{~Hz}, 1 \mathrm{H}), 4.79(\mathrm{br} \mathrm{s}, 1 \mathrm{H}), 4.55-4.42(\mathrm{~m}, 1 \mathrm{H}), 4.07(\mathrm{~s}$, 
$2 \mathrm{H}), 4.06-4.03(\mathrm{~m}, 1 \mathrm{H}), 3.60(\mathrm{~s}, 3 \mathrm{H}), 3.59-3.41(\mathrm{~m}, 2 \mathrm{H}), 2.74(\mathrm{dd}, J$ $=13.8,5.8 \mathrm{~Hz}, 1 \mathrm{H}), 2.66(\mathrm{dd}, J=13.8,7.6 \mathrm{~Hz}, 1 \mathrm{H}), 1.35(\mathrm{~s}, 9 \mathrm{H}) .{ }^{13} \mathrm{C}$ NMR $\left(101 \mathrm{MHz}\right.$, DMSO- $\left.d_{6}\right) \delta 171.4,170.9,168.8,155.6,140.3,132.0$, 131.4, 131.3, 130.5, 127.6, 78.6, 62.3, 57.1, 52.5, 52.5, 34.1, 32.8, 28.6. Methyl-(4S,7S)-7-((tert-butoxycarbonyl)amino)-6,10-dioxo$1,3,4,5,6,7,8,10$-octahydrobenzo[j][1] oxa[8] thia[5]azacyclododecine-4-carboxylate (23b). Compound $23 \mathrm{~b}$ was prepared following the procedure described for the synthesis of compound 23a using 22b (350 mg, $0.76 \mathrm{mmol}, 1.0$ equiv), triphenylphosphine (300 mg, $1.14 \mathrm{mmol}, 1.5$ equiv), and di-tert-butyl azodicarboxylate (263 $\mathrm{mg}, 1.14 \mathrm{mmol}, 1.5$ equiv). The crude product was purified by flash chromatography on a silica gel column using $40-60 \%$ EtOAc in hexane as the eluent to give $23 \mathrm{~b}$ (146 mg, $0.33 \mathrm{mmol}, 44 \%)$ as a colorless powder. HRMS (ESI) $m / z$ calcd for $\mathrm{C}_{20} \mathrm{H}_{26} \mathrm{~N}_{2} \mathrm{NaO}_{7} \mathrm{~S}[\mathrm{M}+\mathrm{Na}]^{+}$ 461.1358, found 461.1356. ${ }^{1} \mathrm{H}$ NMR $\left(400 \mathrm{MHz}\right.$, DMSO- $\left.d_{6}\right) \delta 9.01(\mathrm{~d}, J$ $=9.3 \mathrm{~Hz}, 1 \mathrm{H}), 7.74(\mathrm{~d}, J=7.8 \mathrm{~Hz}, 1 \mathrm{H}), 7.51(\mathrm{td}, J=7.8,1.5 \mathrm{~Hz}, 1 \mathrm{H})$, $7.45-7.33(\mathrm{~m}, 2 \mathrm{H}), 7.12(\mathrm{~d}, J=8.3 \mathrm{~Hz}, 1 \mathrm{H}), 4.77(\mathrm{td}, J=10.4,4.0 \mathrm{~Hz}$, $1 \mathrm{H}), 4.60-4.52(\mathrm{~m}, 1 \mathrm{H}), 4.50-4.35(\mathrm{~m}, 2 \mathrm{H}), 4.29(\mathrm{dd}, J=10.7,4.6$ $\mathrm{Hz}, 1 \mathrm{H}), 3.84(\mathrm{~d}, J=10.3 \mathrm{~Hz}, 1 \mathrm{H}), 3.66(\mathrm{~s}, 3 \mathrm{H}), 3.08(\mathrm{dd}, J=14.5,4.0$ $\mathrm{Hz}, 1 \mathrm{H}), 2.70$ (dd, $J=14.5,10.9 \mathrm{~Hz}, 1 \mathrm{H}), 1.37(\mathrm{~s}, 9 \mathrm{H}) .{ }^{13} \mathrm{C}$ NMR $(101$ $\left.\mathrm{MHz}, \mathrm{DMSO}-d_{6}\right) \delta 171.4,170.1,167.6,155.3,137.3,132.9,132.4$, 131.6, 130.4, 128.1, 78.9, 65.8, 52.7, 52.6, 52.3, 35.9, 33.9, 28.6.

Methyl-(4S,7S)-7-((S)-1-acetylpyrrolidine-2-carboxamido)-6, 10dioxo-1,3,4,5,6,7,8,10-octahydrobenzo[j][1]oxa[8]thia[5]azacyclododecine-4-carboxylate (7). Compound 7 was prepared following the procedure described for the synthesis of compound 6 using 23b (100 mg, $0.22 \mathrm{mmol}, 1.0$ equiv), Ac-L-Pro-OH (69 mg, 0.44 $\mathrm{mmol}, 2.0$ equiv), $\mathrm{EDC} \cdot \mathrm{HCl}(84 \mathrm{mg}, 0.44 \mathrm{mmol}, 2.0$ equiv), and DIPEA ( $39 \mu \mathrm{L}, 0.22 \mathrm{mmol}, 1.0$ equiv). The crude product was purified by reverse-phase HPLC using a gradient from 30 to $75 \%$ acetonitrile in water to give 7 (62 mg, $0.13 \mathrm{mmol}, 59 \%)$ as a colorless powder. HRMS (ESI) $\mathrm{m} / z$ calcd for $\mathrm{C}_{22} \mathrm{H}_{28} \mathrm{~N}_{3} \mathrm{O}_{7} \mathrm{~S}[\mathrm{M}+\mathrm{H}]^{+} 478.1648$, found 478.1651. ${ }^{1} \mathrm{H} \mathrm{NMR}\left(400 \mathrm{MHz}, \mathrm{CDCl}_{3}\right) \delta 7.79(\mathrm{dd}, J=7.7,1.4 \mathrm{~Hz}, 1 \mathrm{H})$, $7.61(\mathrm{~d}, J=7.7 \mathrm{~Hz}, 1 \mathrm{H}), 7.43(\mathrm{td}, J=7.5,1.4 \mathrm{~Hz}, 1 \mathrm{H}), 7.34-7.26(\mathrm{~m}$, $2 \mathrm{H}), 7.20(\mathrm{~d}, J=7.5 \mathrm{~Hz}, 1 \mathrm{H}), 4.83(\mathrm{td}, J=7.3,4.6 \mathrm{~Hz}, 1 \mathrm{H}), 4.69-4.58$ $(\mathrm{m}, 2 \mathrm{H}), 4.56-4.48(\mathrm{~m}, 2 \mathrm{H}), 4.15(\mathrm{~d}, J=11.1 \mathrm{~Hz}, 1 \mathrm{H}), 4.06(\mathrm{~d}, J=11.1$ $\mathrm{Hz}, 1 \mathrm{H}), 3.74(\mathrm{~s}, 3 \mathrm{H}), 3.71-3.61(\mathrm{~m}, 1 \mathrm{H}), 3.52-3.40(\mathrm{~m}, 1 \mathrm{H}), 3.21$ (dd, $J=14.3,4.8 \mathrm{~Hz}, 1 \mathrm{H}), 3.14(\mathrm{dd}, J=14.3,7.1 \mathrm{~Hz}, 1 \mathrm{H}), 2.33-2.21$ $(\mathrm{m}, 1 \mathrm{H}), 2.13-1.96(\mathrm{~m}, 3 \mathrm{H}), 2.06(\mathrm{~s}, 3 \mathrm{H}) .{ }^{13} \mathrm{C}$ NMR $(101 \mathrm{MHz}$, $\left.\mathrm{CDCl}_{3}\right) \delta 171.2,171.0,170.3,168.6,168.6,137.5,132.3,131.2,131.1$, $130.1,127.6,65.0,60.2,52.8,52.5,52.5,48.3,36.3,34.5,28.4,25.0$, 22.4 .

2,2,2-Trichloroethyl 2-((4R,7R)-7-(Hydroxymethyl)-4-(methoxycarbonyl)-11,11-dimethyl-6,9-dioxo-10-oxa-2-thia-5,8diazadodecyl)benzoate (21c). Compound $21 \mathrm{c}$ was prepared following the procedure described for the synthesis of compound 21a using $20 \mathrm{a}(2.01 \mathrm{~g}, 5.81 \mathrm{mmol}, 1.0$ equiv), Boc-D-Ser-OH (1.78 g, $8.72 \mathrm{mmol}$, 1.5 equiv), and $\mathrm{EDC} \cdot \mathrm{HCl}(1.64 \mathrm{~g}, 8.72 \mathrm{mmol}, 1.5$ equiv). The crude reaction mixture was purified by flash chromatography on a silica gel column using $0-5 \% \mathrm{MeOH}$ in DCM as the eluent to give $21 \mathrm{c}(2.55 \mathrm{~g}$, $4.34 \mathrm{mmol}, 75 \%$ ) as a colorless oil. HRMS (ESI) $\mathrm{m} / z$ calcd for $\mathrm{C}_{22} \mathrm{H}_{29} \mathrm{Cl}_{3} \mathrm{~N}_{2} \mathrm{NaO}_{8} \mathrm{~S}[\mathrm{M}+\mathrm{Na}]^{+}$609.0608, found 609.0612. ${ }^{1} \mathrm{H}$ NMR $\left(400 \mathrm{MHz}, \mathrm{CDCl}_{3}\right) \delta 8.07(\mathrm{dd}, J=8.3,1.4 \mathrm{~Hz}, 1 \mathrm{H}), 7.53-7.48(\mathrm{~m}$, $1 \mathrm{H}), 7.45-7.38(\mathrm{~m}, 1 \mathrm{H}), 7.39-7.33(\mathrm{~m}, 2 \mathrm{H}), 5.67(\mathrm{~d}, J=7.3 \mathrm{~Hz}, 1 \mathrm{H})$, $5.00-4.92(\mathrm{~m}, 2 \mathrm{H}), 4.78(\mathrm{ddd}, J=8.3,6.4,5.0 \mathrm{~Hz}, 1 \mathrm{H}), 4.23(\mathrm{~d}, J=$ $13.1 \mathrm{~Hz}, 1 \mathrm{H}), 4.11(\mathrm{~d}, J=13.0 \mathrm{~Hz}, 1 \mathrm{H}), 4.08-4.01(\mathrm{~m}, 1 \mathrm{H}), 3.71(\mathrm{~s}$, $3 \mathrm{H}), 3.68-3.65(\mathrm{~m}, 1 \mathrm{H}), 3.29-3.22(\mathrm{~m}, 1 \mathrm{H}), 2.91(\mathrm{dd}, J=14.0,5.0$ $\mathrm{Hz}, 1 \mathrm{H}), 2.86(\mathrm{dd}, J=14.0,6.4 \mathrm{~Hz}, 1 \mathrm{H}), 2.10(\mathrm{br} \mathrm{s}, 1 \mathrm{H}), 1.42(\mathrm{~s}, 9 \mathrm{H})$. ${ }^{13} \mathrm{C}$ NMR $\left(101 \mathrm{MHz}, \mathrm{CDCl}_{3}\right) \delta 171.4,171.0,165.2,156.0,140.5$, 133.0, 131.8, 131.4, 127.7, 127.6, 94.9, 80.5, 74.6, 63.0, 55.5, 52.8, 52.0, 34.7, 33.4, 28.3.

2-((4R,7R)-7-(Hydroxymethyl)-4-(methoxycarbonyl)-11,11-dimethyl-6,9-dioxo-10-oxa-2-thia-5,8-diazadodecyl)benzoic Acid (22c). Compound $22 \mathrm{c}$ was prepared following the procedure described for the synthesis of compound 22a using 21c (1.58 g, $2.69 \mathrm{mmol}, 1.0$ equiv) and $\mathrm{Zn}$ dust $(1.75 \mathrm{~g}, 26.9 \mathrm{mmol}, 10$ equiv). The crude reaction mixture was purified by flash chromatography on a silica gel column using $0-15 \% \mathrm{MeOH} / \mathrm{EtOAc}$ as the eluent to give $22 \mathrm{c}(834 \mathrm{mg}, 1.83$ mmol, $68 \%$ ) as a colorless powder. HRMS (ESI) $\mathrm{m} / z$ calcd for $\mathrm{C}_{20} \mathrm{H}_{29} \mathrm{~N}_{2} \mathrm{O}_{8} \mathrm{~S}[\mathrm{M}+\mathrm{H}]^{+}$457.1645, found 457.1633. ${ }^{1} \mathrm{H}$ NMR (400 MHz, DMSO- $\left.d_{6}\right) \delta 12.86($ br s, $1 \mathrm{H}), 8.26(\mathrm{~d}, J=7.9 \mathrm{~Hz}, 1 \mathrm{H}), 7.81(\mathrm{dd}$,
$J=8.2,1.5 \mathrm{~Hz}, 1 \mathrm{H}), 7.45(\mathrm{td}, J=7.5,1.5 \mathrm{~Hz}, 1 \mathrm{H}), 7.37-7.26(\mathrm{~m}, 2 \mathrm{H})$, $6.62(\mathrm{~d}, J=8.3 \mathrm{~Hz}, 1 \mathrm{H}), 4.50-4.43(\mathrm{~m}, 1 \mathrm{H}), 4.07(\mathrm{~s}, 2 \mathrm{H}), 4.04-3.99$ $(\mathrm{m}, 1 \mathrm{H}), 3.59(\mathrm{~s}, 3 \mathrm{H}), 3.58-3.43(\mathrm{~m}, 2 \mathrm{H}), 2.74(\mathrm{dd}, J=13.8,5.8 \mathrm{~Hz}$, $1 \mathrm{H}), 2.65(\mathrm{dd}, J=13.8,7.5 \mathrm{~Hz}, 1 \mathrm{H}), 1.34(\mathrm{~s}, 9 \mathrm{H})$. The $\mathrm{CH}_{2} \mathrm{O}$ Hproton was not detectable in this spectrum. ${ }^{13} \mathrm{C}$ NMR $\left(101 \mathrm{MHz}\right.$, DMSO- $\left.d_{6}\right) \delta$ $171.4,170.9,168.8,155.6,140.3,132.0,131.4,131.3,130.6,127.6,79.6$, 78.6, 62.3, 57.1, 52.5, 34.1, 32.8, 28.6.

Methyl-(4R,7R)-7-((tert-butoxycarbonyl)amino)-6,10-dioxo$1,3,4,5,6,7,8,10$-octahydrobenzo[j][1] oxa[8] thia [5]azacyclododecine-4-carboxylate (23c). Compound $23 \mathrm{c}$ was prepared following the procedure described for the synthesis of compound 23a using $22 \mathrm{c}$ ( $500 \mathrm{mg}, 1.09 \mathrm{mmol}, 1.0$ equiv), triphenylphosphine (429 $\mathrm{mg}, 1.64 \mathrm{mmol}, 1.5$ equiv), and di-tert-butyl azodicarboxylate $(377 \mathrm{mg}$, $1.64 \mathrm{mmol}, 1.5$ equiv). The crude product was purified by flash chromatography on a silica gel column using $40-60 \%$ EtOAc in hexane as the eluent to give $23 \mathrm{c}(253 \mathrm{mg}, 0.57 \mathrm{mmol}, 53 \%)$ as a colorless powder. HRMS (ESI) $\mathrm{m} / z$ calcd for $\mathrm{C}_{20} \mathrm{H}_{26} \mathrm{~N}_{2} \mathrm{NaO}_{7} \mathrm{~S}[\mathrm{M}+\mathrm{Na}]^{+}$ 461.1358, found 461.1349. ${ }^{1} \mathrm{H}$ NMR $\left(400 \mathrm{MHz}, \mathrm{CDCl}_{3}\right) \delta 7.79(\mathrm{~d}, J=$ $7.5 \mathrm{~Hz}, 1 \mathrm{H}), 7.42(\mathrm{td}, J=7.5,1.3 \mathrm{~Hz}, 1 \mathrm{H}), 7.36-7.22(\mathrm{~m}, 2 \mathrm{H}), 6.96(\mathrm{~d}$, $J=7.0 \mathrm{~Hz}, 1 \mathrm{H}), 5.51(\mathrm{~d}, J=6.5 \mathrm{~Hz}, 1 \mathrm{H}), 4.92-4.85(\mathrm{~m}, 1 \mathrm{H}), 4.72-$ $4.60(\mathrm{~m}, 2 \mathrm{H}), 4.47-4.38(\mathrm{~m}, 1 \mathrm{H}), 4.14-4.02(\mathrm{~m}, 2 \mathrm{H}), 3.75(\mathrm{~s}, 3 \mathrm{H})$, $3.15(\mathrm{dd}, J=14.4,4.7 \mathrm{~Hz}, 1 \mathrm{H}), 3.05(\mathrm{dd}, J=14.4,5.9 \mathrm{~Hz}, 1 \mathrm{H}), 1.45(\mathrm{~s}$, $9 \mathrm{H}) .{ }^{13} \mathrm{C}$ NMR $\left(101 \mathrm{MHz}, \mathrm{CDCl}_{3}\right) \delta 170.4,169.1,167.8,154.9,137.2$, 132.2, 131.3, 131.0, 130.2, 127.7, 80.4, 65.3, 52.9, 52.6, 51.9, 35.9, 34.8, 28.3.

Methyl-(4R,7R)-7-((S)-1-acetylpyrrolidine-2-carboxamido)-6,10dioxo-1,3,4,5,6,7,8,10-octahydrobenzo[j][1] oxa[8] thia[5]azacyclododecine-4-carboxylate (8). Compound 8 was prepared following the procedure described for the synthesis of compound 6 using $23 \mathrm{c}$ (95 mg, $0.21 \mathrm{mmol}, 1.0$ equiv), Ac-L-Pro-OH (66 mg, 0.42 $\mathrm{mmol}, 2.0$ equiv), $\mathrm{EDC} \cdot \mathrm{HCl}(80 \mathrm{mg}, 0.42 \mathrm{mmol}, 2.0$ equiv), and DIPEA ( $47 \mu \mathrm{L}, 0.27 \mathrm{mmol}, 1.0$ equiv). The crude product was purified by reverse-phase HPLC using a gradient from 30 to $75 \%$ acetonitrile in water to give 8 ( $45 \mathrm{mg}, 94 \mu \mathrm{mol}, 45 \%)$ as a colorless powder. HRMS (ESI) $\mathrm{m} / z$ calcd for $\mathrm{C}_{22} \mathrm{H}_{28} \mathrm{~N}_{3} \mathrm{O}_{7} \mathrm{~S}[\mathrm{M}+\mathrm{H}]^{+} 478.1648$, found 478.1648. ${ }^{1} \mathrm{H}$ NMR $\left(400 \mathrm{MHz}, \mathrm{CDCl}_{3}\right) \delta 7.78(\mathrm{dd}, J=7.7,1.5 \mathrm{~Hz}, 1 \mathrm{H})$, $7.60(\mathrm{~d}, J=7.0 \mathrm{~Hz}, 1 \mathrm{H}), 7.42(\mathrm{td}, J=7.5,1.4 \mathrm{~Hz}, 1 \mathrm{H}), 7.34-7.27(\mathrm{~m}$, $3 \mathrm{H}), 4.87$ (ddd, $J=7.4,6.6,4.5 \mathrm{~Hz}, 1 \mathrm{H}), 4.75-4.64(\mathrm{~m}, 2 \mathrm{H}), 4.60-$ $4.42(\mathrm{~m}, 2 \mathrm{H}), 4.18(\mathrm{~s}, 2 \mathrm{H}), 3.75(\mathrm{~s}, 3 \mathrm{H}), 3.64-3.58(\mathrm{~m}, 1 \mathrm{H}), 3.55-$ $3.37(\mathrm{~m}, 1 \mathrm{H}), 3.19(\mathrm{dd}, J=14.3,4.5 \mathrm{~Hz}, 1 \mathrm{H}), 3.12(\mathrm{dd}, J=14.3,6.6 \mathrm{~Hz}$, $1 \mathrm{H}), 2.34-2.28(\mathrm{~m}, 1 \mathrm{H}), 2.20-2.06(\mathrm{~m}, 1 \mathrm{H}), 2.10(\mathrm{~s}, 3 \mathrm{H}), 2.04-1.91$ $(\mathrm{m}, 2 \mathrm{H}) .{ }^{13} \mathrm{C}$ NMR $\left(101 \mathrm{MHz}^{\left.-\mathrm{CDCl}_{3}\right)} \delta 171.4,170.8,170.5,168.7\right.$, 168.0, 137.4, 132.2, 131.2, 131.1, 130.3, 127.6, 65.0, 59.9, 52.8, 52.4, $52.0,48.3,36.3,34.6,28.3,25.0,22.5$.

2,2,2-Trichloroethyl 2-((4S,7R)-7-(Hydroxymethyl)-4-(methoxycarbonyl)-11,11-dimethyl-6,9-dioxo-10-oxa-2-thia-5,8diazadodecyl)benzoate (21d). Compound 21d was prepared following the procedure described for the synthesis of compound 21a using $20 \mathrm{~b}$ ( $1.30 \mathrm{~g}, 3.25 \mathrm{mmol}, 1.0$ equiv), Boc-D-Ser-OH (990 mg, 4.87 mmol, 1.5 equiv), and $\mathrm{EDC} \cdot \mathrm{HCl}$ ( $932 \mathrm{mg}, 4.88 \mathrm{mmol}, 1.5$ equiv). The crude reaction mixture was purified by flash chromatography on a silica gel column using $0-5 \% \mathrm{MeOH}$ in DCM as the eluent to give $21 \mathrm{~d}(1.37$ $\mathrm{g}, 2.34 \mathrm{mmol}, 72 \%$ ) as a colorless oil. HRMS (ESI) $\mathrm{m} / z$ calcd for $\mathrm{C}_{22} \mathrm{H}_{29} \mathrm{Cl}_{3} \mathrm{~N}_{2} \mathrm{O}_{8} \mathrm{SNa}[\mathrm{M}+\mathrm{Na}]^{+} 609.0608$, found $609.0594 .{ }^{1} \mathrm{H}$ NMR $\left(400 \mathrm{MHz}, \mathrm{CDCl}_{3}\right) \delta 8.09(\mathrm{dd}, J=7.7,1.5 \mathrm{~Hz}, 1 \mathrm{H}), 7.52(\mathrm{td}, J=7.5,1.4$ $\mathrm{Hz}, 1 \mathrm{H}), 7.41-7.36(\mathrm{~m}, 2 \mathrm{H}), 7.30(\mathrm{~d}, J=7.0 \mathrm{~Hz}, 1 \mathrm{H}), 5.57(\mathrm{~d}, J=7.5$ $\mathrm{Hz}, 1 \mathrm{H}), 4.98(\mathrm{~s}, 2 \mathrm{H}), 4.83-4.77(\mathrm{~m}, 1 \mathrm{H}), 4.31-4.20(\mathrm{~m}, 2 \mathrm{H}), 4.10(\mathrm{~d}$, $J=12.8 \mathrm{~Hz}, 1 \mathrm{H}), 4.08-4.01(\mathrm{~m}, 1 \mathrm{H}), 3.73(\mathrm{~s}, 3 \mathrm{H}), 3.68(\mathrm{dd}, J=11.4$, $5.2 \mathrm{~Hz}, 1 \mathrm{H}), 2.96(\mathrm{dd}, J=14.1,4.7 \mathrm{~Hz}, 1 \mathrm{H}), 2.88(\mathrm{dd}, J=14.1,6.2 \mathrm{~Hz}$, $1 \mathrm{H}), 2.79(\mathrm{br} \mathrm{s}, 1 \mathrm{H}), 1.44(\mathrm{~s}, 9 \mathrm{H}) .{ }^{13} \mathrm{C}$ NMR $\left(101 \mathrm{MHz}, \mathrm{CDCl}_{3}\right) \delta$ $171.2,170.9,165.3,155.8,140.4,133.0,131.8,131.4,127.7,127.6,94.9$, $80.5,74.7,63.3,55.2,52.8,52.0,34.9,33.6,28.3$.

2-((4S,7R)-7-(Hydroxymethyl)-4-(methoxycarbonyl)-11,11-dimethyl-6,9-dioxo-10-oxa-2-thia-5,8-diazadodecyl)benzoic Acid (22d). Compound $22 \mathrm{~d}$ was prepared following the procedure described for the synthesis of compound 22a using $21 \mathrm{~d}$ ( $1.05 \mathrm{~g}$, $1.78 \mathrm{mmol}, 1.0$ equiv) and $\mathrm{Zn}$ dust ( $1.16 \mathrm{~g}, 17.8 \mathrm{mmol}, 10$ equiv). The crude reaction mixture was purified by flash chromatography on a silica gel column using $0-20 \% \mathrm{MeOH} / \mathrm{EtOAc}$ as the eluent to give $22 \mathrm{~d}$ (478 mg, 1.05 mmol, 59\%) as a colorless powder. HRMS (ESI) $\mathrm{m} / z$ calcd for $\mathrm{C}_{20} \mathrm{H}_{29} \mathrm{~N}_{2} \mathrm{O}_{8} \mathrm{~S}[\mathrm{M}+\mathrm{H}]^{+}$457.1645, found $457.1635 .{ }^{1} \mathrm{H}$ NMR (400 
MHz, DMSO- $\left.d_{6}\right) \delta 8.25(\mathrm{~d}, J=7.7 \mathrm{~Hz}, 1 \mathrm{H}), 7.82(\mathrm{~d}, J=7.7 \mathrm{~Hz}, 1 \mathrm{H})$, $7.37(\mathrm{t}, J=7.7 \mathrm{~Hz}, 1 \mathrm{H}), 7.33-7.19(\mathrm{~m}, 2 \mathrm{H}), 6.69(\mathrm{~d}, J=7.9 \mathrm{~Hz}, 1 \mathrm{H})$, $4.53-4.38(\mathrm{~m}, 1 \mathrm{H}), 4.18(\mathrm{~d}, J=13.0 \mathrm{~Hz}, 1 \mathrm{H}), 4.13(\mathrm{~d}, J=13.0 \mathrm{~Hz}, 1 \mathrm{H})$, 4.09-3.96 (m, $1 \mathrm{H}), 3.58(\mathrm{~s}, 3 \mathrm{H}), 3.56-3.41(\mathrm{~m}, 2 \mathrm{H}), 2.77(\mathrm{dd}, J=$ $13.8,5.8 \mathrm{~Hz}, 1 \mathrm{H}), 2.68(\mathrm{dd}, J=13.8,7.2 \mathrm{~Hz}, 1 \mathrm{H}), 1.36(\mathrm{~s}, 9 \mathrm{H})$. The OHprotons were not detectable in this spectrum. ${ }^{13} \mathrm{C}$ NMR $(126 \mathrm{MHz}$, DMSO- $\left.d_{6}\right) \delta 171.4,171.0,168.8,155.7,140.4,132.0,131.5,131.4$, 130.6, 127.7, 78.7, 62.2, 57.2, 52.6, 52.6, 34.1, 32.7, 28.6 .

Methyl-(4S,7R)-7-((tert-butoxycarbonyl)amino)-6,10-dioxo$1,3,4,5,6,7,8,10$-octahydrobenzo[j][1] oxa[8] thia[5]azacyclododecine-4-carboxylate (23d). Compound $23 \mathrm{~d}$ was prepared following the procedure described for the synthesis of compound 23a using 22d ( $401 \mathrm{mg}, 0.87 \mathrm{mmol}, 1.0$ equiv), triphenylphosphine ( $344 \mathrm{mg}, 1.32 \mathrm{mmol}, 1.5$ equiv), and di-tert-butyl azodicarboxylate ( 303 $\mathrm{mg}, 1.32 \mathrm{mmol}, 1.5$ equiv). The crude product was purified by flash chromatography on a silica gel column using $40-65 \%$ EtOAc in hexane as the eluent to give $23 \mathrm{~d}(186 \mathrm{mg}, 0.43 \mathrm{mmol}, 49 \%)$ as a colorless powder. HRMS (ESI) $\mathrm{m} / z$ calcd for $\mathrm{C}_{20} \mathrm{H}_{26} \mathrm{~N}_{2} \mathrm{NaO}_{7} \mathrm{~S}[\mathrm{M}+\mathrm{Na}]^{+}$ 461.1358, found 461.1360. ${ }^{1} \mathrm{H} \mathrm{NMR}\left(400 \mathrm{MHz}, \mathrm{CDCl}_{3}\right) \delta 7.82(\mathrm{~d}, J=$ $7.4 \mathrm{~Hz}, 1 \mathrm{H}), 7.45(\mathrm{t}, J=7.5 \mathrm{~Hz}, 1 \mathrm{H}), 7.35-7.29(\mathrm{~m}, 3 \mathrm{H}), 5.75-5.62$ $(\mathrm{m}, 1 \mathrm{H}), 4.96(\mathrm{dd}, J=11.4,3.0 \mathrm{~Hz}, 1 \mathrm{H}), 4.89-4.79(\mathrm{~m}, 1 \mathrm{H}), 4.60-$ $4.56(\mathrm{~m}, 2 \mathrm{H}), 4.10-4.00(\mathrm{~m}, 2 \mathrm{H}), 3.74(\mathrm{~s}, 3 \mathrm{H}), 3.30-3.25(\mathrm{~m}, 1 \mathrm{H})$, $3.20-3.12(\mathrm{~m}, 1 \mathrm{H}), 1.47(\mathrm{~s}, 9 \mathrm{H}) .{ }^{13} \mathrm{C}$ NMR $\left(101 \mathrm{MHz}, \mathrm{CDCl}_{3}\right) \delta$ $170.4,169.2,168.5,155.0,137.3,132.5,131.4,131.4,129.8,127.6,81.1$, $66.7,55.3,52.8,52.1,36.3,34.9,28.2$.

Methyl-(4S,7R)-7-((S)-1-acetylpyrrolidine-2-carboxamido)-6,10dioxo-1,3,4,5,6,7,8,10-octahydrobenzo[j][1] oxa[8] thia[5]azacyclododecine-4-carboxylate (9). Compound 9 was prepared following the procedure described for the synthesis of compound 6 using 23d (120 mg, $0.27 \mathrm{mmol}, 1.0$ equiv), Ac-L-Pro-OH ( $84 \mathrm{mg}, 0.54$ $\mathrm{mmol}, 2.0$ equiv), $\mathrm{EDC} \cdot \mathrm{HCl}(103 \mathrm{mg}, 0.54 \mathrm{mmol}, 2.0$ equiv), and DIPEA ( $47 \mu \mathrm{L}, 0.27 \mathrm{mmol}, 1.0$ equiv). The crude product was purified by reverse-phase HPLC using a gradient from 30 to $75 \%$ acetonitrile in water to give 9 (62 $\mathrm{mg}, 0.13 \mathrm{mmol}, 48 \%$ ) as a colorless powder. HRMS (ESI) $\mathrm{m} / z$ calcd for $\mathrm{C}_{22} \mathrm{H}_{26} \mathrm{~N}_{3} \mathrm{O}_{7} \mathrm{~S}[\mathrm{M}-\mathrm{H}]^{-}$476.1491, found 476.1503. ${ }^{1} \mathrm{H}$ NMR $\left(400 \mathrm{MHz}, \mathrm{CDCl}_{3}\right) \delta 7.89(\mathrm{dd}, J=7.7,1.2 \mathrm{~Hz}, 1 \mathrm{H})$, $7.78(\mathrm{~d}, J=8.8 \mathrm{~Hz}, 1 \mathrm{H}), 7.71(\mathrm{~d}, J=8.8 \mathrm{~Hz}, 1 \mathrm{H}), 7.50-7.42(\mathrm{~m}, 1 \mathrm{H})$, $7.39(\mathrm{dd}, J=7.7,1.2 \mathrm{~Hz}, 1 \mathrm{H}), 7.32(\mathrm{td}, J=7.7,1.5 \mathrm{~Hz}, 1 \mathrm{H}), 5.09(\mathrm{dd}, J=$ $11.1,2.9 \mathrm{~Hz}, 1 \mathrm{H}), 4.91(\mathrm{td}, J=8.9,4.5 \mathrm{~Hz}, 1 \mathrm{H}), 4.88-4.83(\mathrm{~m}, 1 \mathrm{H})$, $4.50-4.37(\mathrm{~m}, 2 \mathrm{H}), 4.30(\mathrm{~d}, J=10.5 \mathrm{~Hz}, 1 \mathrm{H}), 3.74-3.66(\mathrm{~m}, 1 \mathrm{H}), 3.92$ $(\mathrm{d}, J=10.5 \mathrm{~Hz}, 1 \mathrm{H}), 3.71(\mathrm{~s}, 3 \mathrm{H}), 3.53-3.41(\mathrm{~m}, 1 \mathrm{H}), 3.21(\mathrm{dd}, J=$ $14.6,4.5 \mathrm{~Hz}, 1 \mathrm{H}), 2.94(\mathrm{dd}, J=14.6,9.0 \mathrm{~Hz}, 1 \mathrm{H}), 2.41-2.20(\mathrm{~m}, 2 \mathrm{H})$, $2.08(\mathrm{~s}, 3 \mathrm{H}), 2.05-1.87(\mathrm{~m}, 2 \mathrm{H}) .{ }^{13} \mathrm{C}$ NMR $\left(101 \mathrm{MHz}, \mathrm{CDCl}_{3}\right) \delta$ $171.6,171.5,170.8,168.9,167.9,137.2,132.5,132.2,131.8,129.8$, $127.5,66.9,60.2,53.4,52.5,52.4,48.4,36.2,34.8,28.0,25.3,22.6$.

Methyl-(4S,7R)-7-((R)-1-acetylpyrrolidine-2-carboxamido)-6,10dioxo-1,3,4,5,6,7,8,10-octahydrobenzo[j][1]oxa[8] thia[5]azacyclododecine-4-carboxylate (10). Compound 10 was prepared following the procedure described for the synthesis of compound 6 using $23 \mathrm{~d}$ ( $27 \mathrm{mg}, 61 \mu \mathrm{mol}, 1.0$ equiv), Ac-D-Pro-OH (19 mg, 0.12 mmol, 2.0 equiv), $\mathrm{EDC} \cdot \mathrm{HCl}(23 \mathrm{mg}, 0.12 \mathrm{mmol}, 2.0$ equiv), and DIPEA ( $21 \mu \mathrm{L}, 0.12 \mathrm{mmol}, 2.0$ equiv). The crude product was purified by reverse-phase HPLC using a gradient from 30 to $75 \%$ acetonitrile in water to give 10 (16 mg, $33 \mu \mathrm{mol}, 54 \%)$ as a colorless powder. HRMS (ESI) $\mathrm{m} / z$ calcd for $\mathrm{C}_{22} \mathrm{H}_{28} \mathrm{~N}_{3} \mathrm{O}_{7} \mathrm{~S}[\mathrm{M}+\mathrm{H}]^{+}$478.1648, found 478.1663. ${ }^{1} \mathrm{H}$ NMR $\left(400 \mathrm{MHz}, \mathrm{CD}_{3} \mathrm{CN}\right) \delta 8.07(\mathrm{~d}, J=9.0 \mathrm{~Hz}, 1 \mathrm{H})$, $7.96(\mathrm{dd}, J=7.8,1.2 \mathrm{~Hz}, 1 \mathrm{H}), 7.55(\mathrm{td}, J=7.8,1.2 \mathrm{~Hz}, 1 \mathrm{H}), 7.46(\mathrm{dd}, J=$ $7.8,1.4 \mathrm{~Hz}, 1 \mathrm{H}), 7.42(\mathrm{td}, J=7.8,1.4 \mathrm{~Hz}, 1 \mathrm{H}), 7.30(\mathrm{~d}, J=7.4 \mathrm{~Hz}, 1 \mathrm{H})$, $4.92(\mathrm{dd}, J=11.3,3.0 \mathrm{~Hz}, 1 \mathrm{H}), 4.84(\mathrm{ddd}, J=11.7,9.0,4.0 \mathrm{~Hz}, 1 \mathrm{H})$, $4.68-4.62(\mathrm{~m}, 1 \mathrm{H}), 4.40(\mathrm{dd}, J=11.4,2.0 \mathrm{~Hz}, 1 \mathrm{H}), 4.35(\mathrm{~d}, J=9.3 \mathrm{~Hz}$, $1 \mathrm{H}), 4.28-4.23(\mathrm{~m}, 1 \mathrm{H}), 3.86(\mathrm{~d}, J=9.3 \mathrm{~Hz}, 1 \mathrm{H}), 3.71(\mathrm{~s}, 3 \mathrm{H}), 3.55-$ $3.43(\mathrm{~m}, 1 \mathrm{H}), 3.25(\mathrm{dd}, J=14.6,4.0 \mathrm{~Hz}, 1 \mathrm{H}), 2.91(\mathrm{dd}, J=14.6,11.7$ $\mathrm{Hz}, 1 \mathrm{H}), 2.30-2.22(\mathrm{~m}, 1 \mathrm{H}), 2.02(\mathrm{~s}, 3 \mathrm{H}), 2.01-1.94(\mathrm{~m}, 4 \mathrm{H}) .{ }^{13} \mathrm{C}$ NMR $\left(101 \mathrm{MHz}, \mathrm{CDCl}_{3}\right) \delta 172.0,171.1,170.7,169.6,168.9,136.4$, $132.3,131.8,130.8,130.7,127.5,67.1,61.1,53.8,52.7,51.6,48.6,34.7$, $34.4,29.3,25.0,22.8$.

Methyl-(4S,7R)-7-((2S,4R)-1-acetyl-4-hydroxypyrrolidine-2-carboxamido)-6, 10-dioxo-1,3,4,5,6,7,8,10-octahydrobenzo[j][1]oxa[8]thia[5]azacyclododecine-4-carboxylate (11). Compound 11 was prepared following the procedure described for the synthesis of compound 6 using $23 \mathrm{~d}$ ( $39 \mathrm{mg}, 89 \mu \mathrm{mol}, 1.0$ equiv), 4-trans-hydroxy-
Ac-L-Pro-OH (31 mg, $0.18 \mathrm{mmol}, 2.0$ equiv), $\mathrm{EDC} \cdot \mathrm{HCl}(35 \mathrm{mg}, 0.18$ mmol, 2.0 equiv), and DIPEA ( $31 \mu \mathrm{L}, 0.18 \mathrm{mmol}, 2.0$ equiv). The crude product was purified by reverse-phase HPLC using a gradient from 20 to $70 \%$ acetonitrile in water to give $11(14 \mathrm{mg}, 28 \mu \mathrm{mol}, 32 \%)$ as a colorless powder. HRMS (ESI) $m / z$ calcd for $\mathrm{C}_{22} \mathrm{H}_{27} \mathrm{~N}_{3} \mathrm{NaO}_{8} \mathrm{~S}[\mathrm{M}+$ $\mathrm{Na}]^{+}$516.1417, found 516.1407. ${ }^{1} \mathrm{H}$ NMR $\left(400 \mathrm{MHz}, \mathrm{CD}_{3} \mathrm{CN}\right) \delta 8.47$ $(\mathrm{d}, J=9.7 \mathrm{~Hz}, 1 \mathrm{H}), 7.98(\mathrm{dd}, J=7.7,1.4 \mathrm{~Hz}, 1 \mathrm{H}), 7.69(\mathrm{~d}, J=7.3 \mathrm{~Hz}$, $1 \mathrm{H}), 7.56(\mathrm{td}, J=7.5,1.4 \mathrm{~Hz}, 1 \mathrm{H}), 7.48(\mathrm{dd}, J=7.5,1.4 \mathrm{~Hz}, 1 \mathrm{H}), 7.41$ $(\mathrm{td}, J=7.7,1.4 \mathrm{~Hz}, 1 \mathrm{H}), 5.02(\mathrm{dd}, J=11.1,2.5 \mathrm{~Hz}, 1 \mathrm{H}), 4.84$ (ddd, $J=$ $11.7,9.7,3.9 \mathrm{~Hz}, 1 \mathrm{H}), 4.77(\mathrm{~d}, J=9.4 \mathrm{~Hz}, 1 \mathrm{H}), 4.57-4.52(\mathrm{~m}, 1 \mathrm{H})$, $4.52-4.48(\mathrm{~m}, 1 \mathrm{H}), 4.46-4.40(\mathrm{~m}, 1 \mathrm{H}), 4.38(\mathrm{dd}, J=11.2,2.2 \mathrm{~Hz}$, $1 \mathrm{H}), 3.85(\mathrm{~d}, J=9.4 \mathrm{~Hz}, 1 \mathrm{H}), 3.75-3.68(\mathrm{~m}, 1 \mathrm{H}), 3.66(\mathrm{~s}, 3 \mathrm{H}), 3.48$ $(\mathrm{dt}, J=11.1,1.7 \mathrm{~Hz}, 1 \mathrm{H}), 3.24(\mathrm{dd}, J=14.8,3.9 \mathrm{~Hz}, 1 \mathrm{H}), 2.95(\mathrm{dd}, J=$ $14.8,11.7 \mathrm{~Hz}, 1 \mathrm{H}$ ), 2.16 (ddd, $J=13.1,8.6,4.5 \mathrm{~Hz}, 2 \mathrm{H}), 2.10-2.02$ (m, $1 \mathrm{H}), 2.00(\mathrm{~s}, 3 \mathrm{H}) .{ }^{13} \mathrm{C}$ NMR $\left(101 \mathrm{MHz}, \mathrm{CD}_{3} \mathrm{CN}\right) \delta 172.6,170.7$, $170.7,169.3,167.1,137.5,132.8,132.6,132.0,129.8,127.7,69.8,66.0$, $58.9,56.4,53.7,53.4,51.9,37.3,37.2,34.5,21.9$.

tert-Butyl-((4S,7R)-4-carbamoyl-6, 10-dioxo-1,3,4,5,6,7,8,10octahydrobenzo[j][1]oxa[8]thia[5]azacyclododecin-7-yl)carbamate (24). Compound 23d ( $84 \mathrm{mg}, 0.19 \mathrm{mmol}, 1.0$ equiv) was dissolved in 1,2-dichloroethane ( $2 \mathrm{~mL}) ; \mathrm{Me}_{3} \mathrm{SnOH}(143 \mathrm{mg}, 0.76$ mmol, 4.0 equiv) was added, and the mixture was stirred for $45 \mathrm{~min}$ at $83{ }^{\circ} \mathrm{C}$. The reaction was allowed to cool to $\mathrm{rt}$, acidified with $1 \mathrm{M}$ aqueous $\mathrm{HCl}$ solution $(5 \mathrm{~mL})$, and extracted with $\mathrm{DCM}(3 \times 25 \mathrm{~mL})$. The combined organic phase was dried over $\mathrm{MgSO}_{4}$, filtered, and then concentrated under reduced pressure, and the obtained crude mixture was dissolved in DMF $(5 \mathrm{~mL})$. HOBt $x \mathrm{H}_{2} \mathrm{O}(39 \mathrm{mg}, 0.29 \mathrm{mmol}, 1.5$ equiv), $\mathrm{EDC} \cdot \mathrm{HCl}(72 \mathrm{mg}, 0.38 \mathrm{mmol}, 2.0$ equiv), and ammonia ( $7 \mathrm{M}$ in $\mathrm{MeOH}, 54 \mu \mathrm{L}, 0.38 \mathrm{mmol}, 2.0$ equiv) were added, and the mixture was stirred for $2 \mathrm{~h}$ at rt. EtOAc $(50 \mathrm{~mL})$ was then added, and the mixture was washed with $1 \mathrm{M}$ aqueous $\mathrm{HCl}$ solution $(25 \mathrm{~mL})$, saturated aqueous $\mathrm{NaHCO}_{3}$ solution $(25 \mathrm{~mL})$, and brine $(25 \mathrm{~mL})$. The organic phase was dried over $\mathrm{MgSO}_{4}$, filtered, and then concentrated under reduced pressure. The crude product was purified by reverse-phase HPLC using a gradient from 35 to $70 \%$ acetonitrile in water to give $\mathbf{2 4}$ (39 $\mathrm{mg}, 91 \mu \mathrm{mol}, 48 \%$ ) as a colorless powder. HRMS (ESI) $\mathrm{m} / z$ calcd for $\mathrm{C}_{19} \mathrm{H}_{25} \mathrm{~N}_{3} \mathrm{NaO}_{6} \mathrm{~S}[\mathrm{M}+\mathrm{Na}]^{+}$446.1362, found 446.1377. ${ }^{1} \mathrm{H}$ NMR $\left(500 \mathrm{MHz}, \mathrm{CD}_{3} \mathrm{OD}\right) \delta 7.93(\mathrm{~d}, J=7.8 \mathrm{~Hz}, 1 \mathrm{H}), 7.50(\mathrm{t}, J=7.5 \mathrm{~Hz}, 1 \mathrm{H})$, $7.42(\mathrm{~d}, J=7.5 \mathrm{~Hz}, 1 \mathrm{H}), 7.36(\mathrm{t}, J=7.8 \mathrm{~Hz}, 1 \mathrm{H}), 5.02-4.96(\mathrm{~m}, 1 \mathrm{H})$, $4.73(\mathrm{dd}, J=10.5,4.5 \mathrm{~Hz}, 1 \mathrm{H}), 4.60(\mathrm{~d}, J=10.0 \mathrm{~Hz}, 1 \mathrm{H}), 4.42(\mathrm{~d}, J=$ $10.0 \mathrm{~Hz}, 1 \mathrm{H}), 4.40-4.36(\mathrm{~m}, 1 \mathrm{H}), 3.83(\mathrm{~d}, J=9.7 \mathrm{~Hz}, 1 \mathrm{H}), 3.29-3.23$ $(\mathrm{m}, 1 \mathrm{H}), 2.94-2.85(\mathrm{dd}, J=14.6,10.8 \mathrm{~Hz}, 1 \mathrm{H}), 1.48(\mathrm{~s}, 9 \mathrm{H})$. The NH protons were not detectable in this spectrum. ${ }^{13} \mathrm{C}$ NMR $(126 \mathrm{MHz}$, $\left.\mathrm{CD}_{3} \mathrm{OD}\right) \delta 173.6,171.4,167.6,157.2,137.5,132.3,132.1,131.7,129.5$, $127.2,80.3,65.7,55.7,53.7,36.8,34.0,27.2$.

(4S,7R)-7-((S)-1-Acetylpyrrolidine-2-carboxamido)-6,10-dioxo$1,3,4,5,6,7,8,10$-octahydrobenzo[j][1] oxa[8] thia[5]azacyclododecine-4-carboxamide (12). Compound 12 was prepared following the procedure described for the synthesis of compound 6 using 24 ( $26 \mathrm{mg}, 62 \mu \mathrm{mol}, 1.0$ equiv), Ac-L-Pro-OH ( $19 \mathrm{mg}, 0.12 \mathrm{mmol}$, 2.0 equiv), $\mathrm{EDC} \cdot \mathrm{HCl}(23 \mathrm{mg}, 0.12 \mathrm{mmol}, 2.0$ equiv), and DIPEA (20 $\mu \mathrm{L}, 0.12 \mathrm{mmol}, 2.0$ equiv). The crude product was purified by reversephase HPLC using a gradient from 20 to $70 \%$ acetonitrile in water to give 12 ( $14 \mathrm{mg}, 32 \mu \mathrm{mol}, 52 \%$ ) as a colorless powder. HRMS (ESI) $\mathrm{m} / z$ calcd for $\mathrm{C}_{21} \mathrm{H}_{26} \mathrm{~N}_{4} \mathrm{O}_{6} \mathrm{~S}[\mathrm{M}+\mathrm{H}]^{+}$463.1651, found 463.1647. ${ }^{1} \mathrm{H}$ NMR $\left(400 \mathrm{MHz}, \mathrm{DMSO}-d_{6}\right) \delta 9.39(\mathrm{~d}, J=5.1 \mathrm{~Hz}, 1 \mathrm{H}), 8.13(\mathrm{~d}, J=9.7 \mathrm{~Hz}$, $1 \mathrm{H}), 7.92(\mathrm{~d}, J=7.5 \mathrm{~Hz}, 1 \mathrm{H}), 7.54(\mathrm{t}, J=7.3 \mathrm{~Hz}, 1 \mathrm{H}), 7.46(\mathrm{~d}, J=7.3$ $\mathrm{Hz}, 1 \mathrm{H}), 7.40(\mathrm{t}, J=7.5 \mathrm{~Hz}, 1 \mathrm{H}), 7.26(\mathrm{br} \mathrm{s}, 1 \mathrm{H}), 6.48(\mathrm{br} \mathrm{s}, 1 \mathrm{H}), 4.80-$ $4.74(\mathrm{~m}, 2 \mathrm{H}), 4.45-4.36(\mathrm{~m}, 2 \mathrm{H}), 4.36-4.30(\mathrm{~m}, 2 \mathrm{H}), 3.79(\mathrm{~d}, J=9.2$ $\mathrm{Hz}, 1 \mathrm{H}), 3.63-3.53(\mathrm{~m}, 1 \mathrm{H}), 3.52-3.45(\mathrm{~m}, 1 \mathrm{H}), 3.17(\mathrm{dd}, J=14.7$, $3.8 \mathrm{~Hz}, 1 \mathrm{H}), 2.77(\mathrm{dd}, J=14.7,12.1 \mathrm{~Hz}, 1 \mathrm{H}), 2.11-2.01(\mathrm{~m}, 2 \mathrm{H}), 1.95$ $(\mathrm{s}, 3 \mathrm{H}), 1.90-1.81(\mathrm{~m}, 2 \mathrm{H}) .{ }^{13} \mathrm{C}$ NMR $\left(101 \mathrm{MHz}\right.$, DMSO- $\left.d_{6}\right) \delta 174.9$, 172.4, 170.4, 169.0, 167.1, 137.8, 133.3, 133.1, 132.3, 129.6, 128.1, 65.8, $59.7,55.5,54.5,48.5,37.0,34.5,29.2,25.3,22.7$

tert-Butyl-((4S,7R)-4-(methylcarbamoyl)-6,10-dioxo$1,3,4,5,6,7,8,10$-octahydrobenzo[j][1]oxa[8] thia[5]azacyclododecin-7-yl)carbamate (25). Compound 25 was prepared following the procedure described for the synthesis of compound 24 using 23d (136 mg, $0.31 \mathrm{mmol}, 1.0$ equiv), $\mathrm{Me}_{3} \mathrm{SnOH}$ ( $233 \mathrm{mg}, 1.24$ mmol, 4.0 equiv), $\mathrm{HOBt} \cdot x \mathrm{H}_{2} \mathrm{O}(63 \mathrm{mg}, 0.47 \mathrm{mmol}, 1.5$ equiv), EDC. 
$\mathrm{HCl}$ (118 mg, $0.62 \mathrm{mmol}, 2.0$ equiv), and methylamine ( $2 \mathrm{M}$ in THF, $171 \mu \mathrm{L}, 0.34 \mathrm{mmol}, 1.1$ equiv). The crude reaction mixture was purified by flash chromatography on a silica gel column using $0-5 \% \mathrm{MeOH}$ in DCM as the eluent to give $25(78 \mathrm{mg}, 0.18 \mathrm{mmol}, 58 \%)$ as a colorless powder. HRMS (ESI) $m / z$ calcd for $\mathrm{C}_{20} \mathrm{H}_{28} \mathrm{~N}_{3} \mathrm{O}_{6} \mathrm{~S}[\mathrm{M}+\mathrm{H}]^{+} 438.1699$, found 438.1687. ${ }^{1} \mathrm{H}$ NMR $\left(400 \mathrm{MHz}, \mathrm{CDCl}_{3}\right) \delta 7.83(\mathrm{~d}, J=7.8 \mathrm{~Hz}$, $1 \mathrm{H}), 7.48(\mathrm{t}, J=7.5 \mathrm{~Hz}, 1 \mathrm{H}), 7.42-7.31(\mathrm{~m}, 2 \mathrm{H}), 7.19(\mathrm{br} \mathrm{s}, 1 \mathrm{H}), 6.58$ (br s, $1 \mathrm{H}), 5.58(\mathrm{br} \mathrm{s}, 1 \mathrm{H}), 4.90-4.84(\mathrm{~m}, 1 \mathrm{H}), 4.71-4.57,(\mathrm{~m}, 2 \mathrm{H})$, $4.56-4.49(\mathrm{~m}, 1 \mathrm{H}), 4.01(\mathrm{~d}, J=9.8 \mathrm{~Hz}, 1 \mathrm{H}), 3.80(\mathrm{~d}, J=9.8 \mathrm{~Hz}, 1 \mathrm{H})$, $3.37(\mathrm{dd}, J=14.8,5.3 \mathrm{~Hz}, 1 \mathrm{H}), 2.97(\mathrm{dd}, J=14.8,9.8 \mathrm{~Hz}, 1 \mathrm{H}), 2.80(\mathrm{~d}, J$ $=4.1 \mathrm{~Hz}, 3 \mathrm{H}), 1.46(\mathrm{~s}, 9 \mathrm{H}) .{ }^{13} \mathrm{C} \mathrm{NMR}\left(126 \mathrm{MHz}, \mathrm{CDCl}_{3}\right) \delta 170.6$, 170.4, 168.4, 156.4, 136.2, 132.7, 132.1, 131.6, 129.9, 127.9, 81.7, 66.2, $55.9,50.9,34.4,33.6,28.2,26.4$.

(4S,7R)-7-((S)-1-Acetylpyrrolidine-2-carboxamido)- $N$-methyl6,10-dioxo-1,3,4,5,6,7,8,10-octahydrobenzo[j][1] oxa[8] thia[5]azacyclododecine-4-carboxamide (13). Compound 13 was prepared following the procedure described for the synthesis of compound 6 using 25 ( $50 \mathrm{mg}, 0.11 \mathrm{mmol}, 1.0$ equiv), Ac-L-Pro-OH (34 mg, 0.22 mmol, 2.0 equiv), $\mathrm{EDC} \cdot \mathrm{HCl}(42 \mathrm{mg}, 0.22 \mathrm{mmol}, 2.0$ equiv), and DIPEA ( $19 \mu \mathrm{L}, 0.11 \mathrm{mmol}, 1.0$ equiv). The crude product was purified by reverse-phase HPLC using a gradient from 20 to $70 \%$ acetonitrile in water to give 13 (17 mg, $37 \mu \mathrm{mol}, 34 \%$ ) as a colorless powder. HRMS (ESI) $m / z$ calcd for $\mathrm{C}_{22} \mathrm{H}_{29} \mathrm{~N}_{4} \mathrm{O}_{6} \mathrm{~S}[\mathrm{M}+\mathrm{H}]^{+} 477.1808$, found 477.1802. ${ }^{1} \mathrm{H} \mathrm{NMR}\left(400 \mathrm{MHz}, \mathrm{CDCl}_{3}\right) \delta 8.23(\mathrm{~d}, J=9.1 \mathrm{~Hz}, 1 \mathrm{H}), 7.82$ $(\mathrm{d}, J=7.5 \mathrm{~Hz}, 1 \mathrm{H}), 7.45(\mathrm{t}, J=7.8 \mathrm{~Hz}, 1 \mathrm{H}), 7.39(\mathrm{~d}, J=7.8 \mathrm{~Hz}, 1 \mathrm{H})$, $7.32(\mathrm{t}, J=7.5 \mathrm{~Hz}, 1 \mathrm{H}), 7.23(\mathrm{~d}, J=7.1 \mathrm{~Hz}, 1 \mathrm{H}), 6.58(\mathrm{br} \mathrm{s}, 1 \mathrm{H}), 5.08-$ $5.01(\mathrm{~m}, 1 \mathrm{H}), 4.77-4.67(\mathrm{~m}, 2 \mathrm{H}), 4.59-4.53(\mathrm{~m}, 1 \mathrm{H}), 4.21-4.13(\mathrm{~m}$, $1 \mathrm{H}), 3.99(\mathrm{~d}, J=9.5 \mathrm{~Hz}, 1 \mathrm{H}), 3.94(\mathrm{~d}, J=9.5 \mathrm{~Hz}, 1 \mathrm{H}), 3.68-3.59(\mathrm{~m}$, $1 \mathrm{H}), 3.59-3.51(\mathrm{~m}, 2 \mathrm{H}), 2.91-2.81(\mathrm{~m}, 1 \mathrm{H}), 2.76(\mathrm{~d}, J=4.0 \mathrm{~Hz}, 3 \mathrm{H})$, 2.32-2.09 (m, 4H), $2.00(\mathrm{~s}, 3 \mathrm{H}) .{ }^{13} \mathrm{C}$ NMR $\left(101 \mathrm{MHz}, \mathrm{CDCl}_{3}\right) \delta$ $173.4,171.1,170.4,169.4,168.5,136.4,132.5,132.4,131.4,130.1$, 127.6, 66.0, 61.0, 54.5, 53.3, 48.6, 34.8, 34.6, 29.2, 26.3, 25.4, 22.4.

tert-Butyl-((4S,7R)-4-(dimethylcarbamoyl)-6,10-dioxo$1,3,4,5,6,7,8,10$-octahydrobenzo[j][1] oxa[8] thia[5]azacyclododecin-7-yl)carbamate (26). Compound 26 was prepared following the procedure described for the synthesis of compound 24, using $23 \mathrm{~d}$ ( $82 \mathrm{mg}, 0.19 \mathrm{mmol}, 1.0$ equiv), $\mathrm{Me}_{3} \mathrm{SnOH}$ (143 mg, 0.76 mmol, 4.0 equiv), $\mathrm{HOBt} \cdot x \mathrm{H}_{2} \mathrm{O}(38 \mathrm{mg}, 0.28 \mathrm{mmol}, 1.5$ equiv), EDC. $\mathrm{HCl}(72 \mathrm{mg}, 0.38 \mathrm{mmol}, 2.0$ equiv), and dimethylamine ( $2 \mathrm{M}$ in THF, $105 \mu \mathrm{L}, 0.21 \mathrm{mmol}, 1.1$ equiv). The crude product was purified by reverse-phase HPLC using a gradient from 20 to $75 \%$ acetonitrile in water to give 26 ( $40 \mathrm{mg}, 89 \mu \mathrm{mol}, 47 \%)$ as a colorless powder. HRMS (ESI) $\mathrm{m} / z$ calcd for $\mathrm{C}_{21} \mathrm{H}_{29} \mathrm{~N}_{3} \mathrm{NaO}_{6} \mathrm{~S}[\mathrm{M}+\mathrm{Na}]^{+} 474.1675$, found 474.1667. ${ }^{1} \mathrm{H}$ NMR $\left(500 \mathrm{MHz}, \mathrm{CDCl}_{3}\right) \delta 7.86(\mathrm{dd}, J=7.8,1.4 \mathrm{~Hz}, 1 \mathrm{H})$, $7.47(\mathrm{td}, J=7.5,1.5 \mathrm{~Hz}, 1 \mathrm{H}), 7.39-7.31(\mathrm{~m}, 3 \mathrm{H}), 5.58-5.50(\mathrm{~m}, 1 \mathrm{H})$, 5.21 (ddd, $J=8.6,6.7,5.0 \mathrm{~Hz}, 1 \mathrm{H}), 5.06(\mathrm{dd}, J=11.3,2.8 \mathrm{~Hz}, 1 \mathrm{H})$, $4.62-4.55(\mathrm{~m}, 1 \mathrm{H}), 4.50(\mathrm{dd}, J=11.3,2.2 \mathrm{~Hz}, 1 \mathrm{H}), 4.10-4.05(\mathrm{~m}$, $1 \mathrm{H}), 3.99(\mathrm{~d}, J=10.2 \mathrm{~Hz}, 1 \mathrm{H}), 3.14-3.09(\mathrm{~m}, 1 \mathrm{H}), 3.11(\mathrm{~s}, 3 \mathrm{H}), 3.03$ (dd, $J=14.7,6.7 \mathrm{~Hz}, 1 \mathrm{H}), 2.95(\mathrm{~s}, 3 \mathrm{H}), 1.49(\mathrm{~s}, 9 \mathrm{H}) .{ }^{13} \mathrm{C}$ NMR $(126$ $\left.\mathrm{MHz}, \mathrm{CDCl}_{3}\right) \delta 169.5,169.2,168.1,155.1,137.1,132.4,131.8,131.6$, 130.0, 127.6, 81.3, 66.1, 55.3, 48.9, 37.6, 37.1, 35.9, 35.5, 28.2.

(4S,7R)-7-((S)-1-Acetylpyrrolidine-2-carboxamido)- $\mathrm{N}, \mathrm{N}$-dimethyl-6,10-dioxo-1,3,4,5,6,7,8,10-octahydrobenzo[j][1] oxa[8]thia[5]azacyclododecine-4-carboxamide (14). Compound 14 was prepared following the procedure described for the synthesis of compound 6 using 26 (38 mg, $84 \mu \mathrm{mol}, 1.0$ equiv), Ac-L-Pro-OH $(27 \mathrm{mg}, 0.17 \mathrm{mmol}$, 2.0 equiv), $\mathrm{EDC} \cdot \mathrm{HCl}(32 \mathrm{mg}, 0.17 \mathrm{mmol}, 2.0$ equiv), and DIPEA ( 27 $\mu \mathrm{L}, 0.17 \mathrm{mmol}, 2.0$ equiv). The crude product was purified by reversephase HPLC using a gradient from 25 to $75 \%$ acetonitrile in water to give 14 (13 $\mathrm{mg}, 26 \mu \mathrm{mol}, 32 \%$ ) as a colorless powder. HRMS (ESI) $\mathrm{m} / z$ calcd for $\mathrm{C}_{23} \mathrm{H}_{31} \mathrm{~N}_{4} \mathrm{O}_{6} \mathrm{~S}[\mathrm{M}+\mathrm{H}]^{+}$491.1964, found 491.1941. ${ }^{1} \mathrm{H}$ NMR $\left(500 \mathrm{MHz}, \mathrm{CDCl}_{3}\right) \delta 7.90(\mathrm{~d}, J=7.3 \mathrm{~Hz}, 2 \mathrm{H}), 7.54(\mathrm{~d}, J=9.0 \mathrm{~Hz}, 1 \mathrm{H})$, $7.39(\mathrm{t}, J=7.4 \mathrm{~Hz}, 1 \mathrm{H}), 7.32(\mathrm{~d}, J=7.4 \mathrm{~Hz}, 1 \mathrm{H}), 7.27(\mathrm{t}, J=7.6 \mathrm{~Hz}$, $1 \mathrm{H}), 5.13-5.01(\mathrm{~m}, 2 \mathrm{H}), 4.87-4.83(\mathrm{~m}, 1 \mathrm{H}), 4.52-4.47(\mathrm{~m}, 2 \mathrm{H}), 4.44$ $(\mathrm{d}, J=11.0 \mathrm{~Hz}, 1 \mathrm{H}), 3.90(\mathrm{~d}, J=11.0 \mathrm{~Hz}, 1 \mathrm{H}), 3.77-3.65(\mathrm{~m}, 1 \mathrm{H})$, $3.50-3.42(\mathrm{~m}, 1 \mathrm{H}), 3.06(\mathrm{~s}, 3 \mathrm{H}), 3.04-2.94(\mathrm{~m}, 2 \mathrm{H}), 2.92(\mathrm{~s}, 3 \mathrm{H})$, 2.35-2.26 (m, 1H), 2.22-2.12 (m, 1H), $2.15(\mathrm{~s}, 3 \mathrm{H}), 1.96-1.84(\mathrm{~m}$, $2 \mathrm{H}) .{ }^{13} \mathrm{C}$ NMR $\left(126 \mathrm{MHz}, \mathrm{CDCl}_{3}\right) \delta 172.0,171.4,169.5,168.7,167.7$, 137.2, 132.6, 132.3, 132.2, 129.6, 127.6, 66.8, 60.0, 53.3, 49.9, 48.4, $38.4,37.1,35.9,35.8,27.6,25.2,22.6$.
tert-Butyl-((4S,7R)-4-(diethylcarbamoyl)-6,10-dioxo$1,3,4,5,6,7,8,10$-octahydrobenzo[j][1] oxa[8] thia[5]azacyclododecin-7-yl)carbamate (27). Compound 27 was prepared following the procedure described for the synthesis of compound 24, using $23 \mathrm{~d}$ ( $153 \mathrm{mg}, 0.35 \mathrm{mmol}, 1.0$ equiv), $\mathrm{Me}_{3} \mathrm{SnOH}(263 \mathrm{mg}, 1.40$ mmol, 4.0 equiv), HOBt $x \mathrm{H}_{2} \mathrm{O}$ ( $71 \mathrm{mg}, 0.53 \mathrm{mmol}, 1.5$ equiv), EDC. $\mathrm{HCl}$ (133 mg, $0.70 \mathrm{mmol}, 2.0$ equiv), and diethylamine ( $39 \mu \mathrm{L}, 0.38$ mmol, 1.1 equiv). The crude reaction mixture was purified by flash chromatography on a silica gel column using $0-10 \% \mathrm{MeOH}$ in DCM as the eluent to give $27(55 \mathrm{mg}, 0.12 \mathrm{mmol}, 33 \%)$ as a colorless oil. HRMS (ESI) $m / z$ calcd for $\mathrm{C}_{23} \mathrm{H}_{34} \mathrm{~N}_{3} \mathrm{O}_{6} \mathrm{~S}[\mathrm{M}+\mathrm{H}]^{+} 480.2168$, found 480.2184. ${ }^{1} \mathrm{H}$ NMR $\left(400 \mathrm{MHz}, \mathrm{CDCl}_{3}\right) \delta 7.80(\mathrm{~d}, J=7.4 \mathrm{~Hz}, 1 \mathrm{H}), 7.41$ $(\mathrm{t}, J=7.4 \mathrm{~Hz}, 1 \mathrm{H}), 7.32(\mathrm{t}, J=7.5 \mathrm{~Hz}, 1 \mathrm{H}), 7.29-7.21(\mathrm{~m}, 2 \mathrm{H}), 5.53-$ $5.46(\mathrm{~m}, 1 \mathrm{H}), 5.18-5.08(\mathrm{~m}, 1 \mathrm{H}), 4.70-4.53(\mathrm{~m}, 2 \mathrm{H}), 4.56-4.45(\mathrm{~m}$, $1 \mathrm{H}), 4.14(\mathrm{~d}, J=10.8 \mathrm{~Hz}, 1 \mathrm{H}), 4.07(\mathrm{~d}, J=10.8 \mathrm{~Hz}, 1 \mathrm{H}), 3.55-3.33(\mathrm{~m}$, $3 \mathrm{H}), 3.33-3.17(\mathrm{~m}, 1 \mathrm{H}), 3.01(\mathrm{dd}, J=14.2,3.4 \mathrm{~Hz}, 1 \mathrm{H}), 2.79(\mathrm{dd}, J=$ $14.2,8.7 \mathrm{~Hz}, 1 \mathrm{H}), 1.44(\mathrm{~s}, 9 \mathrm{H}), 1.24(\mathrm{t}, J=7.0 \mathrm{~Hz}, 3 \mathrm{H}), 1.11(\mathrm{t}, J=7.0$ $\mathrm{Hz}, 3 \mathrm{H}) .{ }^{13} \mathrm{C}$ NMR $\left(126 \mathrm{MHz}, \mathrm{CDCl}_{3}\right) \delta 169.0,169.0,167.8,154.8$, $137.0,132.1,131.3,131.3,130.5,127.7,80.2,65.5,52.8,48.8,42.1$, 40.8, 37.0, 36.1, 28.3, 14.6, 12.8 .

(4S,7R)-7-((S)-1-Acetylpyrrolidine-2-carboxamido)- $N, N$-diethyl6,10-dioxo-1,3,4,5,6,7,8,10-octahydrobenzo[j][1]oxa[8]thia[5]azacyclododecine-4-carboxamide (15). Compound 15 was prepared following the procedure described for the synthesis of compound 6 using 27 ( $35 \mathrm{mg}, 72 \mu \mathrm{mol}, 1.0$ equiv), Ac-L-Pro-OH $(22 \mathrm{mg}, 0.14 \mathrm{mmol}$, 2.0 equiv), EDC. $\mathrm{HCl}(27 \mathrm{mg}, 0.14 \mathrm{mmol}, 2.0$ equiv), and DIPEA ( 24 $\mu \mathrm{L}, 0.14 \mathrm{mmol}, 2.0$ equiv). The crude product was purified by reversephase HPLC using a gradient from 5 to $95 \%$ acetonitrile in water to give 15 (9 mg, $37 \mu \mathrm{mol}, 24 \%$ ) as a colorless powder. HRMS (ESI) $m / z$ calcd for $\mathrm{C}_{25} \mathrm{H}_{35} \mathrm{~N}_{4} \mathrm{O}_{6} \mathrm{~S}[\mathrm{M}+\mathrm{H}]^{+} 519.2277$, found 519.2286. ${ }^{1} \mathrm{H}$ NMR (600 $\mathrm{MHz}, \mathrm{DMSO}-d_{6}$, mixture of two rotamers in the ratio 6:4) $\delta 9.14(\mathrm{~d}, J=$ $3.8 \mathrm{~Hz}, 0.6 \mathrm{H}), 9.13(\mathrm{~d}, J=3.8 \mathrm{~Hz}, 0.4 \mathrm{H}), 8.61(\mathrm{~d}, J=8.2 \mathrm{~Hz}, 0.4 \mathrm{H})$, $8.18(\mathrm{~d}, J=8.2 \mathrm{~Hz}, 0.6 \mathrm{H}), 7.78(\mathrm{t}, J=7.6 \mathrm{~Hz}, 1 \mathrm{H}), 7.53(\mathrm{t}, J=7.8 \mathrm{~Hz}$ $1 \mathrm{H}), 7.46-7.43(\mathrm{~m}, 1 \mathrm{H}), 7.41(\mathrm{t}, J=7.6 \mathrm{~Hz}, 1 \mathrm{H}), 5.04-4.97(\mathrm{~m}, 1 \mathrm{H})$, $4.97-4.92(\mathrm{~m}, 1 \mathrm{H}), 4.63(\mathrm{~d}, J=9.7 \mathrm{~Hz}, 0.6 \mathrm{H}), 4.54-4.50(\mathrm{~m}, 0.6 \mathrm{H})$, $4.48-4.43(\mathrm{~m}, 1.2 \mathrm{H}), 4.38(\mathrm{dd}, J=8.5,3.4 \mathrm{~Hz}, 0.6 \mathrm{H}), 4.33(\mathrm{dd}, J=$ $10.4,4.5 \mathrm{~Hz}, 0.4 \mathrm{H}), 4.17(\mathrm{dd}, J=10.2,4.5 \mathrm{~Hz}, 0.6 \mathrm{H}), 3.89(\mathrm{~d}, J=9.8$ $\mathrm{Hz}, 0.4 \mathrm{H}), 3.85(\mathrm{~d}, J=9.8 \mathrm{~Hz}, 0.6 \mathrm{H}), 3.62-3.51(\mathrm{~m}, 1 \mathrm{H}), 3.49-3.43$ $(\mathrm{m}, 1 \mathrm{H}), 3.41-3.34(\mathrm{~m}, 2 \mathrm{H}), 3.33-3.27(\mathrm{~m}, 1 \mathrm{H}), 3.22-3.18(\mathrm{~m}, 1 \mathrm{H})$, $3.02-2.93(\mathrm{~m}, 1 \mathrm{H}), 2.80(\mathrm{dd}, J=14.6,11.4 \mathrm{~Hz}, 0.4 \mathrm{H}), 2.76(\mathrm{dd}, J=$ $14.6,11.2 \mathrm{~Hz}, 0.6 \mathrm{H}), 2.23-2.15(\mathrm{~m}, 0.4 \mathrm{H}), 2.05-1.98(\mathrm{~m}, 0.6 \mathrm{H}), 1.95$ (s, $1.8 \mathrm{H}), 1.93-1.88(\mathrm{~m}, 1 \mathrm{H}), 1.88-1.71(\mathrm{~m}, 2 \mathrm{H}), 1.76(\mathrm{~s}, 1.2 \mathrm{H}), 1.14$ $(\mathrm{t}, J=7.0 \mathrm{~Hz}, 1.8 \mathrm{H}), 1.11(\mathrm{t}, J=7.0 \mathrm{~Hz}, 1.2 \mathrm{H}), 1.04(\mathrm{t}, J=7.1 \mathrm{~Hz}$, $1.8 \mathrm{H}), 1.02(\mathrm{t}, J=7.1 \mathrm{~Hz}, 1.2 \mathrm{H}) .{ }^{13} \mathrm{C}$ NMR $\left(151 \mathrm{MHz}\right.$, DMSO- $d_{6}$, mixture of two rotamers) $\delta 172.6$ and 172.3 (1C), 169.5 (1C), 169.4 (1C), 168.9 and 168.8 (1C), 167.7 and 167.6 (1C), 137.5 and 137.4 (1C), 133.1 and 133.0 (1C), 132.6 and 132.4 (1C), 131.9 and 131.8 (1C), 130.4 and $130.3(1 \mathrm{C}), 128.2(1 \mathrm{C}), 65.6$ and $65.5(1 \mathrm{C}), 60.2$ and $59.3(1 \mathrm{C}), 50.9$ and $50.7(1 \mathrm{C}), 50.1$ and $49.6(1 \mathrm{C}), 48.1$ and $46.7(1 \mathrm{C})$, 41.7 and $41.6(1 \mathrm{C}), 37.3$ and $37.0(1 \mathrm{C}), 35.4$ and $35.3(1 \mathrm{C}), 32.4$ and 30.3 (1C), 24.7 (1C), 23.0 and 22.9 (1C), 22.7 and $22.4(1 \mathrm{C}), 14.9$ and 14.8 (1C), 13.4 and 13.3 (1C).

Methyl N-((tert-Butoxycarbonyl)-D-seryl)-S-methyl-D-cysteinate (28). D-Cysteine methyl ester hydrochloride $(1.02 \mathrm{~g}, 5.90 \mathrm{mmol}, 1.0$ equiv) was dissolved in DMF (12 mL); methyl iodide (404 $\mu \mathrm{L}, 6.51$ mmol, 1.1 equiv) and DIPEA ( $2.01 \mathrm{~mL}, 11.8 \mathrm{mmol}, 2.0$ equiv) were then added, and the mixture stirred at $\mathrm{rt}$ for $2 \mathrm{~h}$. The mixture was diluted with EtOAc $(100 \mathrm{~mL})$ and washed with saturated aqueous $\mathrm{NaHCO}_{3}$ solution $(2 \times 50 \mathrm{~mL})$ and brine $(100 \mathrm{~mL})$. The organic phase was dried over $\mathrm{MgSO}_{4}$, filtered, and then concentrated under reduced pressure. The obtained crude mixture was dissolved in acetonitrile $(10 \mathrm{~mL})$; BocD-Ser-OH (1.21 g, $5.90 \mathrm{mmol}, 1.0$ equiv) and $\mathrm{EDC} \cdot \mathrm{HCl}(1.12 \mathrm{~g}, 5.90$ mmol, 1.0 equiv) were added, and the reaction was stirred for $1 \mathrm{~h}$ at rt. $\operatorname{EtOAc}(100 \mathrm{~mL})$ was then added, and the mixture was washed with $1 \mathrm{M}$ aqueous $\mathrm{HCl}$ solution $(50 \mathrm{~mL})$, saturated aqueous $\mathrm{NaHCO}_{3}$ solution $(50 \mathrm{~mL})$, and brine $(100 \mathrm{~mL})$. The organic phase was dried over $\mathrm{MgSO}_{4}$, filtered, and then concentrated under reduced pressure. The obtained colorless oil ( $867 \mathrm{mg}, 2.60 \mathrm{mmol}, 44 \%$ over two steps) was employed in the next step without further purification. HRMS (ESI) $m / z$ calcd for $\mathrm{C}_{13} \mathrm{H}_{25} \mathrm{~N}_{2} \mathrm{O}_{6} \mathrm{~S}[\mathrm{M}+\mathrm{H}]^{+} 337.1433$, found $337.1428 .{ }^{1} \mathrm{H}$ 
NMR (400 MHz, $\left.\mathrm{CDCl}_{3}\right) \delta 7.31$ (br s, $\left.1 \mathrm{H}\right), 5.56(\mathrm{~d}, J=6.7 \mathrm{~Hz}, 1 \mathrm{H})$, 4.84-4.74 (m, 1H), $4.23(\mathrm{br} \mathrm{s}, 1 \mathrm{H}), 4.07(\mathrm{~m}, 1 \mathrm{H}), 3.76(\mathrm{~s}, 3 \mathrm{H}), 3.67$ $(\mathrm{dd}, J=11.6,5.3 \mathrm{~Hz}, 1 \mathrm{H}), 3.00(\mathrm{dd}, J=14.0,4.8 \mathrm{~Hz}, 1 \mathrm{H}), 2.90(\mathrm{dd}, J=$ $14.1,6.5 \mathrm{~Hz}, 1 \mathrm{H}), 2.10(\mathrm{~s}, 3 \mathrm{H}), 1.45(\mathrm{~s}, 9 \mathrm{H})$. The $\mathrm{CH}_{2} \mathrm{OH}$ proton was not detectable in this spectrum. ${ }^{13} \mathrm{C} \mathrm{NMR}\left(101 \mathrm{MHz}, \mathrm{CDCl}_{3}\right) \delta 171.3$, $171.1,155.9,80.5,63.0,55.2,52.8,51.6,36.1,28.3,16.0$.

(R)-2-((tert-Butoxycarbonyl)amino)-3-(((S)-1-methoxy-3-(methylthio)-1-oxopropan-2-yl)amino)-3-oxopropyl Benzoate (29). Compound 28 (215 $\mathrm{mg}, 0.64 \mathrm{mmol}, 1.0$ equiv) was dissolved in DCM (10 $\mathrm{mL})$ and cooled to $0{ }^{\circ} \mathrm{C}$. Benzoyl chloride $(148 \mu \mathrm{L}, 1.28 \mathrm{mmol}, 2.0$ equiv), triethylamine (181 $\mu \mathrm{L}, 1.28 \mathrm{mmol}, 2.0$ equiv), and DMAP (15 $\mathrm{mg}, 0.13 \mathrm{mmol}, 0.2$ equiv) were then added, and the reaction was allowed to warm to rt and stirred for $1 \mathrm{~h}$. The mixture was diluted with EtOAc $(100 \mathrm{~mL})$ and washed with saturated aqueous $\mathrm{NaHCO}_{3}$ solution $(2 \times 25 \mathrm{~mL})$ and brine $(25 \mathrm{~mL})$. The organic phase was dried over $\mathrm{MgSO}_{4}$, filtered, and then concentrated under reduced pressure. The crude reaction mixture was purified by flash chromatography on a silica gel column using $50-70 \%$ EtOAc in hexane as the eluent to give $\mathbf{2 9}(237 \mathrm{mg}, 0.54 \mathrm{mmol}, 84 \%)$ as a yellow oil. HRMS (ESI) $m / z$ calcd for $\mathrm{C}_{20} \mathrm{H}_{28} \mathrm{~N}_{2} \mathrm{O}_{7} \mathrm{SNa}[\mathrm{M}+\mathrm{Na}]^{+} 463.1515$, found 463.1523. ${ }^{1} \mathrm{H}$ NMR $\left(400 \mathrm{MHz}, \mathrm{CDCl}_{3}\right) \delta 8.05-7.99(\mathrm{~m}, 2 \mathrm{H})$, $7.55(\mathrm{t}, J=7.7 \mathrm{~Hz}, 1 \mathrm{H}), 7.42(\mathrm{t}, J=7.7 \mathrm{~Hz}, 2 \mathrm{H}), 7.14(\mathrm{~d}, J=7.7 \mathrm{~Hz}$, $1 \mathrm{H}), 5.46(\mathrm{br} \mathrm{s}, 1 \mathrm{H}), 4.85-4.79(\mathrm{~m}, 1 \mathrm{H}), 4.73-4.59(\mathrm{~m}, 2 \mathrm{H}), 4.55(\mathrm{dd}$, $J=10.8,4.1 \mathrm{~Hz}, 1 \mathrm{H}), 3.70(\mathrm{~s}, 3 \mathrm{H}), 2.99(\mathrm{dd}, J=14.1,4.9 \mathrm{~Hz}, 1 \mathrm{H}), 2.92$ (dd, $J=14.0,5.6 \mathrm{~Hz}, 1 \mathrm{H}), 2.04(\mathrm{~s}, 3 \mathrm{H}), 1.44(\mathrm{~s}, 9 \mathrm{H}) .{ }^{13} \mathrm{C}$ NMR $(101$ $\mathrm{MHz}_{\mathrm{CDCl}}$ ) $\delta 170.8,169.1,166.3,155.4,133.3,129.8,129.8,128.4$, $128.4,128.3,80.8,64.6,54.0,52.7,51.8,36.3,28.2,16.1$.

(R)-2-((S)-1-Acetylpyrrolidine-2-carboxamido)-3-(((S)-1-methoxy-3-(methylthio)-1-oxopropan-2-yl)amino)-3-oxopropyl Benzoate (30). Compound $\mathbf{3 0}$ was prepared following the procedure described for the synthesis of compound 6 using $29(200 \mathrm{mg}, 0.46$ mmol, 1.0 equiv), Ac-L-Pro-OH ( $144 \mathrm{mg}, 0.92 \mathrm{mmol}, 2.0$ equiv), EDC. $\mathrm{HCl}$ ( $174 \mathrm{mg}, 0.92 \mathrm{mmol}, 2.0$ equiv), and DIPEA $(0.16 \mathrm{~mL}, 0.92 \mathrm{mmol}$, 2.0 equiv). The crude reaction mixture was purified by flash chromatography on a silica gel column using $0-10 \% \mathrm{MeOH}$ in DCM as the eluent to give $30(92 \mathrm{mg}, 0.19 \mathrm{mmol}, 41 \%)$ as a colorless powder. HRMS (ESI) $\mathrm{m} / z$ calcd for $\mathrm{C}_{22} \mathrm{H}_{29} \mathrm{~N}_{3} \mathrm{NaO}_{7} \mathrm{~S}[\mathrm{M}+\mathrm{Na}]^{+}$ 502.1624, found 502.1616. ${ }^{1} \mathrm{H} \mathrm{NMR}\left(500 \mathrm{MHz}, \mathrm{CDCl}_{3}\right) \delta 8.03(\mathrm{~d}, J=$ $7.8 \mathrm{~Hz}, 2 \mathrm{H}), 7.67(\mathrm{~d}, J=8.2 \mathrm{~Hz}, 1 \mathrm{H}), 7.55-7.44(\mathrm{~m}, 2 \mathrm{H}), 7.36(\mathrm{t}, J=$ $7.7 \mathrm{~Hz}, 2 \mathrm{H}), 4.86-4.80(\mathrm{~m}, 2 \mathrm{H}), 4.70-4.65(\mathrm{~m}, 1 \mathrm{H}), 4.48-4.44(\mathrm{~m}$, $1 \mathrm{H}), 4.43-4.34(\mathrm{~m}, 1 \mathrm{H}), 3.65(\mathrm{~s}, 3 \mathrm{H}), 3.63-3.58(\mathrm{~m}, 1 \mathrm{H}), 3.46-3.38$ (m, $1 \mathrm{H}), 2.96-2.83(\mathrm{~m}, 2 \mathrm{H}), 2.32-2.22(\mathrm{~m}, 1 \mathrm{H}), 2.20-2.10(\mathrm{~m}, 1 \mathrm{H})$, $2.06(\mathrm{~s}, 3 \mathrm{H}), 1.95(\mathrm{~s}, 3 \mathrm{H}), 1.93-1.82(\mathrm{~m}, 2 \mathrm{H}) .{ }^{13} \mathrm{C} \mathrm{NMR}(126 \mathrm{MHz}$, $\left.\mathrm{CDCl}_{3}\right) \delta 171.7,171.4,171.0,168.7,166.4,133.3,130.0,130.0,129.5$, 128.4, 128.4, 64.3, 60.1, 52.7, 52.5, 51.9, 48.4, 35.9, 27.8, 25.2, 22.6, 15.9 .

(R)-2-((S)-1-Acetylpyrrolidine-2-carboxamido)-3-(((S)-1-(dimethylamino)-3-(methylthio)-1-oxopropan-2-yl)amino)-3-oxopropyl Benzoate (16). Compound 30 (54 mg, $0.11 \mathrm{mmol}, 1.0$ equiv) was dissolved in 1,2-dichloroethane ( $3 \mathrm{~mL})$. $\mathrm{Me}_{3} \mathrm{SnOH}(83 \mathrm{mg}, 0.44 \mathrm{mmol}$, 4.0 equiv) was added, and the mixture was stirred for $45 \mathrm{~min}$ at $83^{\circ} \mathrm{C}$. The reaction was allowed to cool to rt, acidified with $1 \mathrm{M}$ aqueous $\mathrm{HCl}$ solution $(5 \mathrm{~mL})$, and extracted with DCM $(3 \times 40 \mathrm{~mL})$. The organic phase was dried over $\mathrm{MgSO}_{4}$, filtered, and then concentrated under reduced pressure. The obtained crude was dissolved in DMF $(5 \mathrm{~mL})$; HOBt $x \mathrm{H}_{2} \mathrm{O}(23 \mathrm{mg}, 0.17 \mathrm{mmol}, 1.5$ equiv), EDC. $\mathrm{HCl}(42 \mathrm{mg}, 0.22$ mmol, 2.0 equiv), and dimethylamine ( $2 \mathrm{M}$ in THF, $110 \mu \mathrm{L}, 0.22$ mmol, 2.0 equiv) were added, and the mixture was stirred for $2 \mathrm{~h}$ at $\mathrm{rt}$. $\operatorname{EtOAc}(30 \mathrm{~mL})$ was then added, and the mixture was washed with $1 \mathrm{M}$ aqueous $\mathrm{HCl}$ solution $(25 \mathrm{~mL})$, saturated aqueous $\mathrm{NaHCO}_{3}$ solution $(25 \mathrm{~mL})$, and brine $(25 \mathrm{~mL})$. The organic phase was dried over $\mathrm{MgSO}_{4}$, filtered, and then concentrated under reduced pressure. The crude product was purified by reverse-phase HPLC using a gradient from 25 to $75 \%$ acetonitrile in water to give $\mathbf{4 5}(9 \mathrm{mg}, 20 \mu \mathrm{mol}, 18 \%$ over two steps) as a colorless powder. HRMS (ESI) $\mathrm{m} / z$ calcd for $\mathrm{C}_{23} \mathrm{H}_{33} \mathrm{~N}_{4} \mathrm{O}_{6} \mathrm{~S}$ $[\mathrm{M}+\mathrm{H}]^{+}$493.2121, found 493.2119. ${ }^{1} \mathrm{H}$ NMR $\left(400 \mathrm{MHz} \mathrm{CDCl}_{3}\right) \delta$ 8.15-8.07 (m, 2H), 8.07-8.02 (m, 1H), 7.59-7.52 (m, 1H), 7.48$7.39(\mathrm{~m}, 3 \mathrm{H}), 5.07-5.00(\mathrm{~m}, 1 \mathrm{H}), 4.89(\mathrm{dd}, J=11.2,4.3 \mathrm{~Hz}, 1 \mathrm{H})$, $4.83-4.77(\mathrm{~m}, 1 \mathrm{H}), 4.54(\mathrm{dd}, J=7.9,2.8 \mathrm{~Hz}, 1 \mathrm{H}), 4.49(\mathrm{dd}, J=11.2$, $3.5 \mathrm{~Hz}, 1 \mathrm{H}), 3.74(\mathrm{ddd}, J=9.8,8.1,3.5 \mathrm{~Hz}, 1 \mathrm{H}), 3.48-3.41(\mathrm{~m}, 1 \mathrm{H})$, $3.09(\mathrm{~s}, 3 \mathrm{H}), 2.93(\mathrm{~s}, 3 \mathrm{H}), 2.85(\mathrm{dd}, J=13.5,6.7 \mathrm{~Hz}, 1 \mathrm{H}), 2.72(\mathrm{dd}, J=$ $13.5,6.3 \mathrm{~Hz}, 1 \mathrm{H}), 2.41-2.33(\mathrm{~m}, 1 \mathrm{H}), 2.18(\mathrm{~s}, 3 \mathrm{H}), 2.02(\mathrm{~s}, 3 \mathrm{H}), 1.97-$ $1.79(\mathrm{~m}, 3 \mathrm{H}) .{ }^{13} \mathrm{C}$ NMR $\left(126 \mathrm{MHz}, \mathrm{CDCl}_{3}\right) \delta 171.8,171.6,169.9$, 168.2, 166.4, 133.2, 130.0, 130.0, 129.5, 128.4, 128.4, 64.5, 59.9, 52.9, 48.3, 48.3, 37.8, 36.9, 35.9, 27.4, 25.2, 22.6, 16.3 .

Methyl-(S)-2-(((2-((tert-butoxycarbonyl)amino)-3-(dimethylamino)-3-oxopropyl)thio)methyl)benzoate (31). Boc-D-Cys-OH (6.81 g, $30.8 \mathrm{mmol}, 1.5$ equiv) was dissolved in DMF $(20 \mathrm{~mL})$ and THF $(80$ $\mathrm{mL}$ ). Methyl 2-bromomethylbenzoate ( $4.72 \mathrm{~g}, 20.5 \mathrm{mmol}, 1.0$ equiv) and triethylamine $(6.38 \mathrm{~mL}, 45.1 \mathrm{mmol}, 2.2$ equiv) were added, and the mixture was stirred for $16 \mathrm{~h}$ at $\mathrm{rt}$. After evaporation of THF under reduced pressure, the mixture was diluted with EtOAc $(250 \mathrm{~mL})$ and washed with $1 \mathrm{M}$ aqueous $\mathrm{HCl}$ solution $(100 \mathrm{~mL})$ and brine $(2 \times 100$ $\mathrm{mL}$ ). The organic phase was dried over $\mathrm{MgSO}_{4}$, filtered, and then concentrated under reduced pressure, and the obtained oil was dissolved in DMF ( $50 \mathrm{~mL}$ ). Dimethylamine hydrochloride $(3.34 \mathrm{~g}, 41.0$ mmol, 2.0 equiv), $\mathrm{EDC} \cdot \mathrm{HCl}(7.86 \mathrm{~g}, 41.0 \mathrm{mmol}, 2.0$ equiv), HOBt $x \mathrm{H}_{2} \mathrm{O}(4.15 \mathrm{~g}, 30.8 \mathrm{mmol}, 1.5$ equiv), and DIPEA $(7.16 \mathrm{~mL}, 41.0 \mathrm{mmol}$, 2.0 equiv) were then added, and the mixture was stirred for 2 additional hours at rt. The mixture was diluted with EtOAc $(250 \mathrm{~mL})$ and washed with $1 \mathrm{M}$ aqueous $\mathrm{HCl}$ solution $(100 \mathrm{~mL})$, saturated aqueous $\mathrm{NaHCO}_{3}$ solution $(100 \mathrm{~mL})$, and brine $(100 \mathrm{~mL})$. The organic phase was dried over $\mathrm{MgSO}_{4}$, filtered, and then concentrated under reduced pressure. The crude reaction mixture was purified by flash chromatography on a silica gel column using $10-100 \%$ EtOAc in hexane as the eluent to give 31 (3.90 g, $9.84 \mathrm{mmol}, 48 \%$ over two steps) as a yellow oil. HRMS (ESI) $\mathrm{m} / z$ calcd for $\mathrm{C}_{19} \mathrm{H}_{29} \mathrm{~N}_{2} \mathrm{O}_{5} \mathrm{~S}[\mathrm{M}+\mathrm{H}]^{+}$397.1797, found 397.1804. ${ }^{1} \mathrm{H}$ NMR $\left(500 \mathrm{MHz}, \mathrm{CDCl}_{3}\right) \delta 7.90(\mathrm{~d}, J=7.7 \mathrm{~Hz}, 1 \mathrm{H}), 7.42$ $(\mathrm{t}, J=7.3 \mathrm{~Hz}, 1 \mathrm{H}), 7.38(\mathrm{~d}, J=7.3 \mathrm{~Hz}, 1 \mathrm{H}), 7.30(\mathrm{t}, J=7.6 \mathrm{~Hz}, 1 \mathrm{H})$, $5.37(\mathrm{~d}, J=8.7 \mathrm{~Hz}, 1 \mathrm{H}), 4.81-4.75(\mathrm{~m}, 1 \mathrm{H}), 4.18-4.08(\mathrm{~m}, 2 \mathrm{H}), 3.89$ $(\mathrm{s}, 3 \mathrm{H}), 3.06(\mathrm{~s}, 3 \mathrm{H}), 2.95(\mathrm{~s}, 3 \mathrm{H}), 2.76(\mathrm{dd}, J=13.7,7.4 \mathrm{~Hz}, 1 \mathrm{H}), 2.63$ $(\mathrm{dd}, J=13.7,6.0 \mathrm{~Hz}, 1 \mathrm{H}), 1.43(\mathrm{~s}, 9 \mathrm{H}) .{ }^{13} \mathrm{C} \mathrm{NMR}\left(126 \mathrm{MHz}, \mathrm{CDCl}_{3}\right) \delta$ 171.0, 167.7, 155.1, 140.2, 131.9, 131.2, 131.2, 129.4, 127.2, 79.8, 52.2, 49.6, 37.4, 35.9, 34.9, 34.6, 28.3.

Methyl-2-((4S,7R)-4-(dimethylcarbamoyl)-7-(2-hydroxyethyl)11,11-dimethyl-6,9-dioxo-10-oxa-2-thia-5,8-diazadodecyl)benzoate (32). Compound 31 (1.18 g, $3.01 \mathrm{mmol}, 1.0$ equiv) was dissolved in $4 \mathrm{M} \mathrm{HCl}$ in dioxane $(10 \mathrm{~mL})$ and stirred for $1 \mathrm{~h}$ at $\mathrm{rt}$. After evaporation of the solvent under reduced pressure, the resulting salt was dissolved in acetonitrile $(15 \mathrm{~mL})$. Boc-D-Homoser-OH (1.99 g, 9.03 mmol, 3.0 equiv), EDC $\cdot \mathrm{HCl}(1.72 \mathrm{~g}, 9.03 \mathrm{mmol}, 3.0$ equiv), and DIPEA ( $524 \mu \mathrm{L}, 3.01 \mathrm{mmol}, 1.0$ equiv) were added, and the reaction was stirred for $2 \mathrm{~h}$ at rt. EtOAc $(100 \mathrm{~mL})$ was then added, and the mixture was washed with $1 \mathrm{M}$ aqueous $\mathrm{HCl}$ solution $(50 \mathrm{~mL})$, saturated aqueous $\mathrm{NaHCO}_{3}$ solution $(50 \mathrm{~mL})$, and brine $(50 \mathrm{~mL})$. The organic phase was dried over $\mathrm{MgSO}_{4}$, filtered, and then concentrated under reduced pressure. The reaction crude was purified by flash chromatography on a silica gel column using $0-10 \% \mathrm{MeOH}$ in $\mathrm{DCM}$ as the eluent to give 32 (500 $\mathrm{mg}, 1.01 \mathrm{mmol}, 33 \%$ over two steps) as a white foam. HRMS (ESI) $m / z$ calcd for $\mathrm{C}_{23} \mathrm{H}_{35} \mathrm{~N}_{3} \mathrm{O}_{7} \mathrm{NaS}[\mathrm{M}+\mathrm{Na}]^{+} 520.2093$, found $520.2079 .{ }^{1} \mathrm{H}$ NMR $\left(500 \mathrm{MHz}, \mathrm{CDCl}_{3}\right) \delta 7.89(\mathrm{dd}, J=7.8,1.2 \mathrm{~Hz}, 1 \mathrm{H})$, $7.44(\mathrm{td}, J=7.7,1.2 \mathrm{~Hz}, 1 \mathrm{H}), 7.37(\mathrm{dd}, J=7.7,1.2 \mathrm{~Hz}, 1 \mathrm{H}), 7.31(\mathrm{td}, J=$ $7.8,1.2 \mathrm{~Hz}, 1 \mathrm{H}), 7.21-7.13(\mathrm{~m}, 1 \mathrm{H}), 5.61(\mathrm{~d}, J=7.4 \mathrm{~Hz}, 1 \mathrm{H}), 5.06-$ $4.99(\mathrm{~m}, 1 \mathrm{H}), 4.40-4.33(\mathrm{~m}, 1 \mathrm{H}), 4.18(\mathrm{~d}, J=13.2 \mathrm{~Hz}, 1 \mathrm{H}), 4.08(\mathrm{~d}, J=$ $13.2 \mathrm{~Hz}, 1 \mathrm{H}), 3.90(\mathrm{~s}, 3 \mathrm{H}), 3.75-3.66(\mathrm{~m}, 2 \mathrm{H}), 3.02(\mathrm{~s}, 3 \mathrm{H}), 2.94(\mathrm{~s}$, $3 \mathrm{H}), 2.80(\mathrm{dd}, J=13.7,6.6 \mathrm{~Hz}, 1 \mathrm{H}), 2.69(\mathrm{dd}, J=13.7,6.4 \mathrm{~Hz}, 1 \mathrm{H})$, 2.04-1.95 (m, 1H), 1.83-1.73 (m, 1H), $1.44(\mathrm{~s}, 9 \mathrm{H})$. The $\mathrm{CH}_{2} \mathrm{OH}$ proton was not detectable in this spectrum. ${ }^{13} \mathrm{C}$ NMR $(126$ $\left.\mathrm{MHz} \mathrm{CDCl}_{3}\right) \delta 171.5,170.2,167.9,156.3,140.0,132.0,131.2,131.2$, $129.5,127.4,80.3,58.8,52.3,52.0,48.6,37.4,36.0,36.0,35.1,34.1$, 28.3.

2-((4S,7R)-4-(Dimethylcarbamoyl)-7-(2-hydroxyethyl)-11,11-dimethyl-6,9-dioxo-10-oxa-2-thia-5,8-diazadodecyl)benzoic Acid (33). Compound 33 ( $480 \mathrm{mg}, 0.97 \mathrm{mmol}, 1.0$ equiv) was dissolved in $\mathrm{MeOH}(5 \mathrm{~mL})$ and $0.1 \mathrm{M}$ aqueous $\mathrm{LiOH}(20 \mathrm{~mL})$, and the reaction mixture was stirred at $35^{\circ} \mathrm{C}$ for $16 \mathrm{~h}$. The reaction was then allowed to cool to $\mathrm{rt}$, acidified with $1 \mathrm{M}$ aqueous $\mathrm{HCl}$ solution $(5 \mathrm{~mL})$, and extracted with DCM $(3 \times 50 \mathrm{~mL})$. The organic phase was dried over $\mathrm{MgSO}_{4}$, filtered, and then concentrated under reduced pressure providing compound 33 (302 $\mathrm{mg}, 0.63 \mathrm{mmol}, 64 \%)$ as a colorless 
solid. HRMS (ESI) $m / z$ calcd for $\mathrm{C}_{22} \mathrm{H}_{34} \mathrm{~N}_{3} \mathrm{O}_{7} \mathrm{~S}[\mathrm{M}+\mathrm{H}]^{+}$484.2117, found 484.2120. ${ }^{1} \mathrm{H}$ NMR $\left(600 \mathrm{MHz}, \mathrm{DMSO}-d_{6}\right) \delta 12.94$ (br s, $\left.1 \mathrm{H}\right)$, $8.01(\mathrm{~d}, J=8.5 \mathrm{~Hz}, 1 \mathrm{H}), 7.84(\mathrm{dd}, J=7.8,1.4 \mathrm{~Hz}, 1 \mathrm{H}), 7.47(\mathrm{td}, J=7.5$, $1.4 \mathrm{~Hz}, 1 \mathrm{H}), 7.40(\mathrm{dd}, J=7.5,1.4 \mathrm{~Hz}, 1 \mathrm{H}), 7.36(\mathrm{td}, J=7.8,1.4 \mathrm{~Hz}$, $1 \mathrm{H}), 6.87(\mathrm{~d}, J=8.0 \mathrm{~Hz}, 1 \mathrm{H}), 4.88-4.82(\mathrm{~m}, 1 \mathrm{H}), 4.53-4.45(\mathrm{~m}, 1 \mathrm{H})$, $4.14(\mathrm{~d}, J=13.0 \mathrm{~Hz}, 1 \mathrm{H}), 4.05(\mathrm{~d}, J=13.0 \mathrm{~Hz}, 1 \mathrm{H}), 4.95-4.03(\mathrm{~m}, 1 \mathrm{H})$, $3.48-3.36(\mathrm{~m}, 2 \mathrm{H}), 2.94(\mathrm{~s}, 3 \mathrm{H}), 2.82(\mathrm{~s}, 3 \mathrm{H}), 2.75(\mathrm{dd}, J=13.6,7.7$ $\mathrm{Hz}, 1 \mathrm{H}), 2.46(\mathrm{dd}, J=13.6,5.9 \mathrm{~Hz}, 1 \mathrm{H}), 1.80-1.71(\mathrm{~m}, 1 \mathrm{H}), 1.69-$ $1.58(\mathrm{~m}, 1 \mathrm{H}), 1.38(\mathrm{~s}, 9 \mathrm{H}) .{ }^{13} \mathrm{C}$ NMR $\left(151 \mathrm{MHz}\right.$, DMSO- $\left.d_{6}\right) \delta 172.3$, $170.1,168.8,155.7,140.6,131.9,131.4,131.3,130.6,127.6,78.6,58.0$, 52.4, 48.6, 37.0, 35.7, 35.4, 34.3, 33.4, 28.6.

tert-Butyl-((4S,7R)-4-(dimethylcarbamoyl)-6,11-dioxo1,4,5,6,7,8,9,11-octahydro-3H-benzo[c][1]oxa[6]thia[9]azacyclotridecin-7-yl)carbamate (34). Compound 34 was prepared following the procedure described for the synthesis of compound 23a using 33 (163 $\mathrm{mg}, 0.33 \mathrm{mmol}, 1.0$ equiv), triphenylphosphine (173 mg, $0.66 \mathrm{mmol}, 2.0$ equiv), and di-tert-butyl azodicarboxylate ( $152 \mathrm{mg}, 0.66$ mmol, 2.0 equiv). The crude product was purified by reverse-phase HPLC using a gradient from 20 to $70 \%$ acetonitrile in water to give 34 (32 $\mathrm{mg}, 69 \mu \mathrm{mol}, 21 \%$ ) as a colorless powder. HRMS (ESI) $\mathrm{m} / z$ calcd for $\mathrm{C}_{22} \mathrm{H}_{32} \mathrm{~N}_{3} \mathrm{O}_{6} \mathrm{~S}[\mathrm{M}+\mathrm{H}]^{+}$466.2012. found 466.2003. ${ }^{1} \mathrm{H}$ NMR (500 $\left.\mathrm{MHz}, \mathrm{CDCl}_{3}\right) \delta 7.67(\mathrm{~d}, J=7.2 \mathrm{~Hz}, 1 \mathrm{H}), 7.46-7.38(\mathrm{~m}, 2 \mathrm{H}), 7.33-$ $7.27(\mathrm{~m}, 1 \mathrm{H}), 7.22(\mathrm{~d}, J=7.9 \mathrm{~Hz}, 1 \mathrm{H}), 5.74(\mathrm{~d}, J=9.2 \mathrm{~Hz}, 1 \mathrm{H}), 4.92$ $(\mathrm{td}, J=8.5,2.7 \mathrm{~Hz}, 1 \mathrm{H}), 4.70-4.63(\mathrm{~m}, 1 \mathrm{H}), 4.59-4.52(\mathrm{~m}, 1 \mathrm{H})$, $4.42-4.43(\mathrm{~m}, 1 \mathrm{H}), 4.21(\mathrm{~d}, J=13.6 \mathrm{~Hz}, 1 \mathrm{H}), 3.98(\mathrm{~d}, J=13.6 \mathrm{~Hz}, 1 \mathrm{H})$, $2.98(\mathrm{~s}, 3 \mathrm{H}), 2.93(\mathrm{~s}, 3 \mathrm{H}), 2.82-2.70(\mathrm{~m}, 2 \mathrm{H}), 2.39(\mathrm{dd}, J=14.2,8.5$ $\mathrm{Hz}, 1 \mathrm{H}), 2.08-1.99(\mathrm{~m}, 1 \mathrm{H}), 1.48$ (s, 9H). ${ }^{13} \mathrm{C} \mathrm{NMR}(126 \mathrm{MHz}$, $\left.\mathrm{CDCl}_{3}\right) \delta 170.6,169.5,168.9,156.4,138.9,131.9,131.7,131.6,129.4$, 127.4, 80.8, 63.7, 51.0, 49.5, 37.1, 36.0, 34.2, 32.8, 30.5, 28.4 .

(4S,7R)-7-((S)-1-Acetylpyrrolidine-2-carboxamido)- $N, N$-dimethyl-6,11-dioxo-1,4,5,6,7,8,9,11-octahydro-3H-benzo[c][1]oxa[6]thia[9]azacyclotridecine-4-carboxamide (17). Compound 17 was prepared following the procedure described for the synthesis of compound 6 using 34 ( $25 \mathrm{mg}, 54 \mu \mathrm{mol}, 1.0$ equiv), Ac-L-Pro-OH ( $25 \mathrm{mg}$, 0.16 $\mathrm{mmol}, 3.0$ equiv), $\mathrm{EDC} \cdot \mathrm{HCl}(31 \mathrm{mg}, 0.16 \mathrm{mmol}, 3.0$ equiv), and DIPEA ( $28 \mu \mathrm{L}, 0.16 \mathrm{mmol}, 3.0$ equiv). The crude product was purified by reverse-phase HPLC using a gradient from 20 to $70 \%$ acetonitrile in water to give 17 ( $13 \mathrm{mg}, 25 \mu \mathrm{mol}, 46 \%$ ) as a colorless powder. HRMS (ESI) $\mathrm{m} / z$ calcd for $\mathrm{C}_{24} \mathrm{H}_{33} \mathrm{~N}_{4} \mathrm{O}_{6} \mathrm{~S}[\mathrm{M}+\mathrm{H}]^{+}$505.2121, found 505.2121. ${ }^{1} \mathrm{H} \mathrm{NMR}\left(600 \mathrm{MHz}, \mathrm{CDCl}_{3}\right) \delta 8.12(\mathrm{~d}, J=9.9 \mathrm{~Hz}, 1 \mathrm{H}), 7.73$ $(\mathrm{d}, J=7.6 \mathrm{~Hz}, 1 \mathrm{H}), 7.57(\mathrm{~d}, J=6.9 \mathrm{~Hz}, 1 \mathrm{H}), 7.55(\mathrm{~d}, J=7.7 \mathrm{~Hz}, 1 \mathrm{H})$, $7.44(\mathrm{t}, J=7.7 \mathrm{~Hz}, 1 \mathrm{H}), 7.29-7.25(\mathrm{~m}, 1 \mathrm{H}), 4.89-4.83(\mathrm{~m}, 2 \mathrm{H}), 4.75$ $(\mathrm{td}, J=11.5,2.5 \mathrm{~Hz}, 1 \mathrm{H}), 4.58-4.55(\mathrm{~m}, 1 \mathrm{H}), 4.54(\mathrm{~d}, J=13.9 \mathrm{~Hz}, 1 \mathrm{H})$, $4.32(\mathrm{dt}, J=12.2,4.3 \mathrm{~Hz}, 1 \mathrm{H}), 3.90-3.85(\mathrm{~m}, 1 \mathrm{H}), 3.82(\mathrm{~d}, J=13.9 \mathrm{~Hz}$, $1 \mathrm{H}), 3.53(\mathrm{td}, J=9.4,6.6 \mathrm{~Hz}, 1 \mathrm{H}), 2.93(\mathrm{~s}, 6 \mathrm{H}), 2.90-2.86(\mathrm{~m}, 1 \mathrm{H})$, $2.65-2.56(\mathrm{~m}, 2 \mathrm{H}), 2.44-2.38(\mathrm{~m}, 1 \mathrm{H}), 2.36-2.28(\mathrm{~m}, 1 \mathrm{H}), 2.13(\mathrm{~s}$, $3 \mathrm{H}), 2.11-2.03(\mathrm{~m}, 2 \mathrm{H}), 2.00-1.93(\mathrm{~m}, 1 \mathrm{H}) .{ }^{13} \mathrm{C}$ NMR $(151 \mathrm{MHz}$, $\left.\mathrm{CDCl}_{3}\right) \delta 172.0,171.6,170.2,169.3,169.1,139.2,132.2,131.5,131.2$, 130.1, 127.0, 61.5, 60.7, 50.8, 49.4, 47.7, 36.8, 35.8, 32.7, 31.7, 29.3, 28.7, 24.9, 22.6.

Methyl-(S)-2-(4-(dimethylcarbamoyl)-11,11-dimethyl-6,9-dioxo10-oxa-2-thia-5,8-diazadodecyl)benzoate (35). Compound 31 (4.16 $\mathrm{g}, 10.5 \mathrm{mmol}, 1.0$ equiv) was dissolved in $4 \mathrm{M} \mathrm{HCl}$ in dioxane $(15 \mathrm{~mL})$ and stirred for $1 \mathrm{~h}$ at $\mathrm{rt}$. After evaporation of the solvent under reduced pressure, the resulting salt was dissolved in acetonitrile $(35 \mathrm{~mL})$. BocGly-OH (2.76 g, $15.8 \mathrm{mmol}, 1.5$ equiv), $\mathrm{EDC} \cdot \mathrm{HCl}(3.03 \mathrm{~g}, 15.8 \mathrm{mmol}$, 1.5 equiv), and DIPEA ( $1.83 \mathrm{~mL}, 10.5 \mathrm{mmol}, 1.0$ equiv) were added, and the reaction was stirred for $2 \mathrm{~h}$ at $\mathrm{rt}$. EtOAc $(200 \mathrm{~mL})$ was then added, and the mixture was washed with $1 \mathrm{M}$ aqueous $\mathrm{HCl}$ solution $(100 \mathrm{~mL})$, saturated aqueous $\mathrm{NaHCO}_{3}$ solution $(100 \mathrm{~mL})$, and brine $(50 \mathrm{~mL})$. The organic phase was dried over $\mathrm{MgSO}_{4}$, filtered, and then concentrated under reduced pressure. The crude reaction mixture was purified by flash chromatography on a silica gel column using $0-10 \%$ $\mathrm{MeOH}$ in DCM as the eluent to give $35(3.81 \mathrm{~g}, 8.41 \mathrm{mmol}, 80 \%$ over two steps) as a colorless oil. HRMS (ESI) $m / z$ calcd for $\mathrm{C}_{21} \mathrm{H}_{32} \mathrm{~N}_{3} \mathrm{O}_{6} \mathrm{~S}$ $[\mathrm{M}+\mathrm{H}]^{+} 454.2012$, found 454.2002. ${ }^{1} \mathrm{H}$ NMR $\left(400 \mathrm{MHz} \mathrm{CDCl}_{3}\right) \delta$ $7.90(\mathrm{dd}, J=7.8,1.3 \mathrm{~Hz}, 1 \mathrm{H}), 7.44(\mathrm{td}, J=7.6,1.3 \mathrm{~Hz}, 1 \mathrm{H}), 7.37(\mathrm{dd}, J=$ $7.6,1.3 \mathrm{~Hz}, 1 \mathrm{H}), 7.31(\mathrm{td}, J=7.8,7.3 \mathrm{~Hz}, 1 \mathrm{H}), 6.90(\mathrm{~d}, J=7.1 \mathrm{~Hz}, 1 \mathrm{H})$, $5.24-5.14(\mathrm{~m}, 1 \mathrm{H}), 5.12-5.02(\mathrm{~m}, 1 \mathrm{H}), 4.17(\mathrm{~d}, J=13.1 \mathrm{~Hz}, 1 \mathrm{H}), 4.07$ $(\mathrm{d}, J=13.1 \mathrm{~Hz}, 1 \mathrm{H}), 3.90(\mathrm{~s}, 3 \mathrm{H}), 3.85-3.73(\mathrm{~m}, 2 \mathrm{H}), 3.03(\mathrm{~s}, 3 \mathrm{H})$, $2.94(\mathrm{~s}, 3 \mathrm{H}), 2.80(\mathrm{dd}, J=14.0,6.8 \mathrm{~Hz}, 1 \mathrm{H}), 2.69(\mathrm{dd}, J=14.0,5.6 \mathrm{~Hz}$, $1 \mathrm{H}), 1.44(\mathrm{~s}, 9 \mathrm{H}) .{ }^{13} \mathrm{C} \mathrm{NMR}\left(126 \mathrm{MHz}, \mathrm{CDCl}_{3}\right) \delta 170.3,168.8,167.8$, $155.8,140.1,132.0,131.2,131.2,129.4,127.3,80.2,52.3,48.3,44.1$, $37.4,35.9,35.2,34.2,28.3$.

Methyl 2-((4S,10R)-4-(Dimethylcarbamoyl)-10-(hydroxymethyl)14,14-dimethyl-6,9,12-trioxo-13-oxa-2-thia-5,8, 11 triazapentadecyl)benzoate (36). Compound 35 ( $3.80 \mathrm{~g}, 8.40 \mathrm{mmol}$, 1.0 equiv) was dissolved in $4 \mathrm{M} \mathrm{HCl}$ in dioxane $(15 \mathrm{~mL})$ and stirred for $1 \mathrm{~h}$ at $\mathrm{rt}$. After evaporation of the solvent under reduced pressure, the resulting salt was dissolved in acetonitrile $(25 \mathrm{~mL})$. Boc-D-Ser-OH (3.44 g, $16.8 \mathrm{mmol}, 2.0$ equiv), $\mathrm{EDC} \cdot \mathrm{HCl}(3.21 \mathrm{~g}, 16.8 \mathrm{mmol}, 2.0$ equiv), and DIPEA ( $1.46 \mathrm{~mL}, 8.40 \mathrm{mmol}, 1.0$ equiv) were added, and the reaction was stirred for $2 \mathrm{~h}$ at rt. EtOAc $(150 \mathrm{~mL})$ was then added, and the mixture was washed with $1 \mathrm{M}$ aqueous $\mathrm{HCl}$ solution $(50 \mathrm{~mL})$, saturated aqueous $\mathrm{NaHCO}_{3}$ solution $(50 \mathrm{~mL})$, and brine $(50 \mathrm{~mL})$. The organic phase was dried over $\mathrm{MgSO}_{4}$, filtered, and then concentrated under reduced pressure. The crude reaction mixture was purified by flash chromatography on a silica gel column using $0-15 \% \mathrm{MeOH}$ in $\mathrm{DCM}$ as the eluent to give 36 ( $1.50 \mathrm{~g}, 2.77 \mathrm{mmol}, 33 \%$ over two steps) as a colorless oil. HRMS (ESI) $\mathrm{m} / z$ calcd for $\mathrm{C}_{24} \mathrm{H}_{36} \mathrm{~N}_{4} \mathrm{O}_{8} \mathrm{NaS}[\mathrm{M}+$ $\mathrm{Na}]^{+}$563.2152, found 563.2145. ${ }^{1} \mathrm{H}$ NMR $\left(500 \mathrm{MHz}, \mathrm{CDCl}_{3}\right) \delta 7.89$ $(\mathrm{d}, J=9.4 \mathrm{~Hz}, 1 \mathrm{H}), 7.52(\mathrm{~d}, J=8.2 \mathrm{~Hz}, 1 \mathrm{H}), 7.46-7.42(\mathrm{~m}, 1 \mathrm{H}), 7.36-$ $7.29(\mathrm{~m}, 2 \mathrm{H}), 7.22-7.15(\mathrm{~m}, 1 \mathrm{H}), 5.62(\mathrm{~d}, J=8.6 \mathrm{~Hz}, 1 \mathrm{H}), 5.06-4.95$ $(\mathrm{m}, 1 \mathrm{H}), 4.32-4.22(\mathrm{~m}, 2 \mathrm{H}), 4.12-4.03(\mathrm{~m}, 3 \mathrm{H}), 3.92-3.90(\mathrm{~m}, 1 \mathrm{H})$, $3.90(\mathrm{~s}, 3 \mathrm{H}), 3.71-3.60(\mathrm{~m}, 1 \mathrm{H}), 3.03(\mathrm{~s}, 3 \mathrm{H}), 2.91(\mathrm{~s}, 3 \mathrm{H}), 2.75(\mathrm{dd}, J$ $=13.9,7.2 \mathrm{~Hz}, 1 \mathrm{H}), 2.66(\mathrm{dd}, J=13.9,6.1 \mathrm{~Hz}, 1 \mathrm{H}), 1.43(\mathrm{~s}, 9 \mathrm{H}) .{ }^{13} \mathrm{C}$ NMR $\left(126 \mathrm{MHz}, \mathrm{CDCl}_{3}\right) \delta 172.0,170.7,168.5,168.0,155.6,140.0$, $132.1,131.2,131.2,129.5,127.4,80.3,63.2,55.8,52.3,48.4,43.1,37.5$, $36.1,35.3,33.9,28.3$.

2-((4S, 10R)-4-(Dimethylcarbamoyl)-10-(hydroxymethyl)-14,14dimethyl-6,9, 12-trioxo-13-oxa-2-thia-5,8,11-triazapentadecyl)benzoic Acid (37). Compound 36 (502 mg, $0.93 \mathrm{mmol}, 1.0$ equiv) was dissolved in $\mathrm{MeOH}(5 \mathrm{~mL})$ and $0.1 \mathrm{M}$ aqueous $\mathrm{LiOH}(20 \mathrm{~mL})$. After being stirred at $35{ }^{\circ} \mathrm{C}$ for $16 \mathrm{~h}$, the reaction was acidified with $1 \mathrm{M}$ aqueous $\mathrm{HCl}$ solution $(5 \mathrm{~mL})$ and extracted with $\mathrm{DCM}(3 \times 50 \mathrm{~mL})$. The organic phase was dried over $\mathrm{MgSO}_{4}$, filtered, and then concentrated under reduced pressure. The crude reaction mixture was purified by flash chromatography on a silica gel column using $0-$ $15 \% \mathrm{MeOH}$ in DCM as the eluent to give $37(240 \mathrm{mg}, 0.46 \mathrm{mmol}$, $49 \%$ ) as a colorless powder. HRMS (ESI) $m / z$ calcd for $\mathrm{C}_{23} \mathrm{H}_{33} \mathrm{~N}_{4} \mathrm{O}_{8} \mathrm{~S}$ $[\mathrm{M}-\mathrm{H}]^{-}$525.2019, found 525.2023. ${ }^{1} \mathrm{H}$ NMR $\left(500 \mathrm{MHz}\right.$, DMSO- $\left.d_{6}\right)$ $\delta 12.77(\mathrm{br} \mathrm{s}, 1 \mathrm{H}), 8.20(\mathrm{~d}, J=8.3 \mathrm{~Hz}, 1 \mathrm{H}), 8.16-8.04(\mathrm{~m}, 1 \mathrm{H}), 7.83$ $(\mathrm{dd}, J=7.6,1.4 \mathrm{~Hz}, 1 \mathrm{H}), 7.48(\mathrm{td}, J=7.6,1.3 \mathrm{~Hz}, 1 \mathrm{H}), 7.40(\mathrm{dd}, J=7.6$, $1.3 \mathrm{~Hz}, 1 \mathrm{H}), 7.35(\mathrm{td}, J=7.6,1.4 \mathrm{~Hz}, 1 \mathrm{H}), 6.72(\mathrm{~d}, J=7.8 \mathrm{~Hz}, 1 \mathrm{H})$, $4.88-4.70(\mathrm{~m}, 1 \mathrm{H}), 4.13(\mathrm{~d}, J=13.0 \mathrm{~Hz}, 1 \mathrm{H}), 4.09(\mathrm{~d}, J=13.0 \mathrm{~Hz}, 1 \mathrm{H})$, $4.01-3.96(\mathrm{~m}, 1 \mathrm{H}), 3.79-3.65(\mathrm{~m}, 2 \mathrm{H}), 3.56(\mathrm{~d}, J=5.5 \mathrm{~Hz}, 2 \mathrm{H}), 2.96$ (s, 3H), $2.83(\mathrm{~s}, 3 \mathrm{H}), 2.75(\mathrm{dd}, J=13.5,7.9 \mathrm{~Hz}, 1 \mathrm{H}), 2.48-2.42(\mathrm{~m}$, $1 \mathrm{H}), 1.39$ (s, 9H). ${ }^{13} \mathrm{C}$ NMR (126 MHz, DMSO-d $d_{6} \delta 171.2,170.0$, $169.0,168.8,155.7,140.5,131.9,131.4,131.4,131.2$, 127.6, 78.7, 62.4, 57.1, 48.7, 42.3, 37.1, 35.8, 34.4, 33.6, 28.6.

tert-Butyl-((4S, 10R)-4-(dimethylcarbamoyl)-6,9,13-trioxo1,4,5,6,7,8,9,10,11,13-decahydro-3H-benzo[m][1]oxa[11]thia[5,8]diazacyclopentadecin-10-yl)carbamate (38). Compound 38 was prepared following the procedure described for the synthesis of compound 23a using 37 (130 mg, $0.25 \mathrm{mmol}, 1.0$ equiv), triphenylphosphine $(131 \mathrm{mg}, 0.50 \mathrm{mmol}, 2.0$ equiv), and di-tert-butyl azodicarboxylate ( $115 \mathrm{mg}, 0.50 \mathrm{mmol}, 2.0$ equiv). The crude product was purified by reverse-phase HPLC using a gradient from 20 to $70 \%$ acetonitrile in water to give $\mathbf{3 8}(25 \mathrm{mg}, 50 \mu \mathrm{mol}, 20 \%)$ as a colorless powder. HRMS (ESI) $m / z$ calcd for $\mathrm{C}_{23} \mathrm{H}_{33} \mathrm{~N}_{4} \mathrm{O}_{7} \mathrm{~S}[\mathrm{M}+\mathrm{H}]^{+}$509.2070, found 509.2068. ${ }^{1} \mathrm{H}$ NMR $\left(500 \mathrm{MHz}, \mathrm{CDCl}_{3}\right) \delta 7.72(\mathrm{~d}, J=7.5 \mathrm{~Hz}$, $1 \mathrm{H}), 7.56-7.47(\mathrm{~m}, 1 \mathrm{H}), 7.41-7.36(\mathrm{~m}, 1 \mathrm{H}), 7.36-7.26(\mathrm{~m}, 3 \mathrm{H}), 5.87$ $(\mathrm{d}, J=6.7 \mathrm{~Hz}, 1 \mathrm{H}), 4.93-4.86(\mathrm{~m}, 1 \mathrm{H}), 4.84(\mathrm{~d}, J=11.0 \mathrm{~Hz}, 1 \mathrm{H})$, $4.69-4.61(\mathrm{~m}, 1 \mathrm{H}), 4.39(\mathrm{dd}, J=11.1,5.7 \mathrm{~Hz}, 1 \mathrm{H}), 4.23-4.13(\mathrm{~m}$, $1 \mathrm{H}), 4.09(\mathrm{~d}, J=13.3 \mathrm{~Hz}, 1 \mathrm{H}), 3.93(\mathrm{~d}, J=13.6 \mathrm{~Hz}, 1 \mathrm{H}), 3.63-3.55(\mathrm{~m}$, $1 \mathrm{H}), 3.08(\mathrm{~s}, 3 \mathrm{H}), 2.93(\mathrm{~s}, 3 \mathrm{H}), 2.84-2.75(\mathrm{~m}, 1 \mathrm{H}), 2.66-2.55(\mathrm{~m}$, $1 \mathrm{H}), 1.45(\mathrm{~s}, 9 \mathrm{H}) .{ }^{13} \mathrm{C} \mathrm{NMR}\left(126 \mathrm{MHz} \mathrm{CDCl}_{3}\right) \delta 170.1,169.9,168.8$, $167.4,155.3,139.1,131.7,130.7,130.5,130.2$, 127.4, 80.5, 65.2, 53.1, $48.8,43.7,37.3,36.1,35.4,33.9,28.3$. 
(4S, 10R)-10-((S)-1-Acetylpyrrolidine-2-carboxamido)- $\mathrm{N}, \mathrm{N}$-dimethyl-6,9,13-trioxo-1,4,5,6,7,8,9,10,11,13-decahydro-3H-benzo[m][1]oxa[11]thia[5,8]diazacyclopentadecine-4-carboxamide (18). Compound 18 was prepared following the procedure described for the synthesis of compound 6 using 38 ( $20 \mathrm{mg}, 39 \mu \mathrm{mol}, 1.0$ equiv), Ac-LPro-OH ( $19 \mathrm{mg}, 0.12 \mathrm{mmol}, 3.0$ equiv), $\mathrm{EDC} \cdot \mathrm{HCl}(23 \mathrm{mg}, 0.12 \mathrm{mmol}$, 3.0 equiv), and DIPEA ( $21 \mu \mathrm{L}, 0.12 \mathrm{mmol}, 3.0$ equiv). The crude product was purified by reverse-phase HPLC using a gradient from 25 to $75 \%$ acetonitrile in water to give $\mathbf{1 8}(9 \mathrm{mg}, 16 \mu \mathrm{mol}, 41 \%)$ as a colorless powder. HRMS (ESI) $m / z$ calcd for $\mathrm{C}_{25} \mathrm{H}_{34} \mathrm{~N}_{5} \mathrm{O}_{7} \mathrm{~S}[\mathrm{M}+\mathrm{H}]^{+}$ 548.2179, found 548.2128. ${ }^{1} \mathrm{H} \mathrm{NMR}\left(500 \mathrm{MHz}, \mathrm{CDCl}_{3}\right) \delta 8.20(\mathrm{dd}, J=$ $7.6,4.7 \mathrm{~Hz}, 1 \mathrm{H}), 7.88(\mathrm{dd}, J=7.8,1.5 \mathrm{~Hz}, 1 \mathrm{H}), 7.59(\mathrm{~d}, J=9.3 \mathrm{~Hz}, 1 \mathrm{H})$, $7.50(\mathrm{~d}, J=7.7 \mathrm{~Hz}, 1 \mathrm{H}), 7.47(\mathrm{td}, J=7.5,1.5 \mathrm{~Hz}, 1 \mathrm{H}), 7.40$ (dd, $J=7.7$, $1.3 \mathrm{~Hz}, 1 \mathrm{H}), 7.36(\mathrm{td}, J=7.5,1.4 \mathrm{~Hz}, 1 \mathrm{H}), 5.18(\mathrm{dd}, J=11.3,2.3 \mathrm{~Hz}$, $1 \mathrm{H}), 4.94(\mathrm{dt}, J=9.3,2.8 \mathrm{~Hz}, 1 \mathrm{H}), 4.86(\mathrm{td}, \mathrm{J}=8.1,4.6 \mathrm{~Hz}, 1 \mathrm{H}), 4.47$ $(\mathrm{dd}, J=11.2,3.3 \mathrm{~Hz}, 1 \mathrm{H}), 4.37-4.25(\mathrm{~m}, 3 \mathrm{H}), 3.82(\mathrm{~d}, J=13.5 \mathrm{~Hz}$, $1 \mathrm{H}), 3.72-3.66(\mathrm{~m}, 1 \mathrm{H}), 3.62-3.49(\mathrm{~m}, 2 \mathrm{H}), 3.13(\mathrm{~s}, 3 \mathrm{H}), 2.94(\mathrm{~s}$, $3 \mathrm{H}), 2.89-2.80(\mathrm{~m}, 2 \mathrm{H}), 2.29-2.14(\mathrm{~m}, 3 \mathrm{H}), 2.12(\mathrm{~s}, 3 \mathrm{H}), 2.03-1.94$ (m, 1H). ${ }^{13} \mathrm{C}$ NMR $\left(126 \mathrm{MHz}, \mathrm{CDCl}_{3}\right) \delta 172.7,170.6,170.4,169.1$, 168.9, 168.2, 139.1, 132.2, 131.4, 130.8, 129.7, 127.6, 64.3, 61.2 52.4, 48.6, 48.0, 44.0, 37.4, 36.2, 36.1, 34.9, 30.0, 25.4, 22.5.

Calculation of Descriptors and PCA Analysis. Two-dimensional structures from the DNP and DrugBank ${ }^{16}$ databases were imported into MOE (version 2015.10, Chemical Computing Group, www. chemcomp.com).$^{49}$ Both data sets were cleaned by addition of explicit hydrogen atoms, removal of organometallic compounds, and adjustment of protonation states (i.e., strong acids were deprotonated and strong bases were protonated). Duplicate structures were eliminated using the 2D SMILES string of each compound. Molecular descriptors were calculated for all compounds in the DNP and in DrugBank, and for the 41 lead-like macrocycle cores identified from the DNP, using the MOE descriptor module. ${ }^{50}$ Principal component analysis (PCA) was performed using SIMCA (version 14.1) (o $^{51}$ to identify clusters and investigate the coverage of the chemical space of the DNP and DrugBank.

Extraction of Macrocyclic Cores from the DNP. The structures of the natural products contained in the sd-file version of the Dictionary of Natural Products (DNP) were cleaned as described previously, whereby natural products containing toxic or reactive groups are removed using the knowledge-based HTS filter in Pipeline Pilot. ${ }^{12}$ Further filtering in Pipeline Pilot was performed to retain macrocycles ( $\geq 12$ and $\leq 30$ atoms in the ring) having $<10$ rings in total and a MW $<$ $2500 \mathrm{Da}$ to provide 3657 macrocyclic natural products.

The side chains of the macrocyclic natural products were then pruned to provide macrocycle cores using the first two steps of the fragment generation algorithm reported previously, ${ }^{12}$ i.e., deglycosylation and extraction of extended Murcko frameworks ${ }^{15}$ decorated with attachment points (Figure S2). In these two steps, macrocycles were not fragmented and all ring systems and linker atoms were kept while linear side chains were pruned, but not removed. This provided macrocycle cores, i.e., macrocycles decorated with attachment points, which are functional groups linked directly to the ring system or pruned side chains. Thus, the algorithm was designed to retain functional groups and only cut side chains at carbon-carbon bonds at a distance of $>2$ atoms from the ring.

The algorithm defines an attachment point by traversing through the side chain of the macrocycle through two heavy atoms, starting from the ring-based atom. For each heavy atom thereafter it decides, based on a set of rules, ${ }^{12}$ whether the atom is kept, whether that atoms neighboring atoms are examined, or if the side chain is cut after the second heavy atom. The algorithm keeps aliphatic carbon atoms as long as they are within two atoms from the ring, and longer aliphatic side chains are pruned to ethyl groups. If the algorithm encounters a carbon atom linked to a non-carbon and non-hydrogen atom, it will keep the heteroatom and examine the immediate neighbor atom, irrespective of its distance from the ring. The algorithm prunes after the next upcoming carbon atom as long as this is not double-bonded to a heteroatom.

In branched side chains, the algorithm traverses the branches separately, within the distance limit. If the branching carbon is connected to a heteroatom by a double bond, the immediate next neighbor atom in the other branch will be investigated using the same rules as for a linear side chain, irrespective of its distance from the ring. The next upcoming carbon atom is kept, as the terminating carbon, but the rest of the side chain is pruned. If the branching atom is a heteroatom, the immediate next neighbor atoms will be investigated in the same manner, irrespective of their distance from the ring. As a result of this pruning process, the original functional group motif of the macrocyclic part of the natural product remains intact in the core. In addition, functional groups close to the macrocycle ring in side chains remain intact. All generated macrocycle cores are stored in a database for further processing.

If identical macrocyclic cores were obtained after side-chain pruning of different natural products, only one was kept. Cores with $\leq 3 \mathrm{O}$ or $\mathrm{N}$ atoms or $>15$ rotatable bonds, without a heteroatom in the macrocycle, and/or having more than one macrocycle were also removed in Pipeline Pilot. This resulted in 2175 natural-product-derived macrocycle cores, which are based on 764 different Murcko scaffolds. The 2175 cores were clustered in Pipeline Pilot using an FCFP6 pharmacophore fingerprint $^{52}$ into 217 clusters (Supporting Excel Sheet). The cluster centers were visually inspected by three or more experienced medicinal chemists, and 41 cluster centers or near neighbors were selected by exclusion of cores containing reactive or potentially redox-sensitive groups, excessively complex structures including $>6$ rings, or oligomeric/pseudo-oligomeric structures (Supporting Excel Sheet).

Docking of Macrocycle Cores into Keap1. The atomic coordinates of four crystal structures of human Keap1, in complex with a small molecule [PDB ID: $4 \operatorname{IQK}^{24}(1.97 \AA)$ and 3VNG $(2.1 \AA)$ ] or in the apo form [PDB ID: 4IFJ (1.8 $)$ and $1 \mathrm{ZGK}^{25}(1.35 \AA)$ ], were obtained from the Protein Data Bank. Each crystal structure was imported and refined with Protein Preparation Wizard ${ }^{53}$ using the Schrödinger Suite. ${ }^{54}$ Structure refinement included adding hydrogen atoms, assigning bond orders, building disulfide bonds, and removal of water molecules beyond $5 \AA$ from the ligand atoms. The PROPKA tool from Protein Preparation Wizard was used to predict the protonation states of the ionizable residues at $\mathrm{pH}$ 7.0. ${ }^{55}$ Subsequently, the positions of the hydrogen atoms in the Keap1 structure were energy-minimized using the OPLS3 force field. ${ }^{56}$

The receptor grid generation module of Glide ${ }^{26}$ was used to define the active site for the docking experiments. The active site of Keap1 was defined either by the bound ligand or by key residues in the active site (R415, R483, Y525, Y572, A556, S603), which were used as the centroid of the grid box (the radius of the active site was $15 \AA$ from the centroid). The docking protocol was first validated by comparing the conformation (the pose) of the bound ligands as obtained from docking with the one determined by X-ray crystallography for the two ligandbound structures (PDB ID: 4IQK ${ }^{24}$ and 3VNG; see the Supporting Information, Computational Procedure 1). Subsequently, the set of 41 cores was preprocessed using LigPrep, which included generating possible ionization states (at $\mathrm{pH} 7$ ) using the Epik tool ${ }^{57}$ and structure minimization using the OPLS3 force field. ${ }^{56}$ During preprocessing, input chirality was retained.

The 41 lead-like macrocyclic cores (Table S1) were then docked into each of the four refined Keap1 structures using Glide, which treats the cores as fully flexible while Keap1 remains rigid during the docking. Docking poses for each macrocycle core were ranked according to the GlideScore from the standard precision mode, and for each core, the docked pose that had the lowest GlideScore for binding to each of the Keap 1 structures was identified (Table S2). The resulting combined list of the top-10 cores for each Keap1 structure was then used to rank the cores by the number of Keap1 structures each core bound to (Table S3), after which the docked poses of the top-five cores in each of the four crystal structure were inspected visually (Figure S5).

Induced-fit docking (IFD) $)^{58}$ of stereoisomeric macrocycles 6-9 was performed as implemented in the Schrödinger suite. ${ }^{58,59}$ Threedimensional structures of compounds 6-9 were built with Maestro from the Schrödinger suite ${ }^{60}$ and energy-minimized using the OPLS3 force field. A grid centered at the bound ligand (PDB ID: $4 \mathrm{IQK}^{24}$ ) with the box size of $15 \AA$ was defined. Subsequently, 6-9 were docked and scored using the standard precision mode with an extended sampling 
protocol, which will generate up to 80 poses per ligand from several docking runs. The cyclothialidine core was included for comparison. A total of 286 binding poses were analyzed with regard to if the phenyl ring of 1 and 6-9 reached deep into the Kelch channel of the Keap1 binding pocket (Figure S6).

Binding Affinity Calculations. The atomic coordinates of Keap 1 in complex with compound $\mathbf{1 4}$ were preprocessed as described above in the docking section. The chloride ion in the ligand binding pocket was included in the binding affinity calculations as it is coordinated with residues in Keap1 (N382, N414, and R415) and with 14. Methyl ester (9), amide (12), monomethyl amide (13), and diethylamide (15) were modeled into the Keap1 binding site using the bound conformation of 14 as a template. For compounds 9 and 13, two alternative conformations of the methyl ester and methyl amide (cis- and transforms) were explored, while five alternative conformations were explored for the diethylamide of $\mathbf{1 5}$ (Tables S6 and S7).

Prior to affinity calculations, the ligand binding site was defined using the receptor grid generation module available in Glide as described in the docking section above. Subsequently, the initial input ligand coordinates for 9 and 12-15 were optimized in the receptor, the ligand binding pose was scored, and poses were used for ligand binding affinity calculations in Prime MM-GBSA ${ }^{34}$ as implemented in the Schrödinger suite. The ligand and a $5 \AA$ region of the protein around the ligand were treated as flexible in the binding affinity calculation. Other settings were chosen as defaults.

Similarity to Reported Inhibitors of the Keap1-Nrf2 PPI. A set of inhibitors of the Keap1-Nrf2 PPI, termed "PubChem", was retrieved from the PubChem Bioassay database (PubChem Bioassay AIDs: 504523; 588683; 651798; 651801; 651806; 651807; 651823; 651829; $651833 ; 651834){ }^{38}$ Duplicate entries, compounds that lacked complete stereochemical information, and solvents were removed from the 1127 compounds reported as active in the 10 listed bioassays. Compounds that did not pass the PAINS filter ${ }^{39}$ were also removed, providing a PubChem set consisting of 375 inhibitors (Table S8). Another set of "validated" inhibitors was assembled from two sources. First, nine compounds, reported to show reproducible activity in a triad of orthogonal assays by Tran et al., ${ }^{20}$ were selected. Second, crystal structures that had a compound bound in the binding site for Nrf2 of Keap1 were retrieved from the Protein Data Bank (PDB). Peptides were excluded, while fragments were retained, providing a validated set of 35 compounds (Table S9).

The Tanimoto coefficient ${ }^{40}$ was calculated to determine the similarity between compound $\mathbf{1 4}$ and the Keap1 inhibitors in the validated and PubChem sets, respectively, using seven commonly used structural fingerprints (Figure S11). The similarity was also calculated based on all fingerprints. All substructure similarity analyses were performed with the Canvas tool (version 3.1.011). ${ }^{61}$ The molecular similarity landscape (a so-called networklike similarity graph) was investigated for the merged validated and PubChem sets and for the validated set alone, using compound $\mathbf{1 4}$ as a reference. The structures of all compounds were imported, and a substructure fragment fingerprint (FragFp)-based similarity landscape was computed using DataWarrior (version 5.2.1). ${ }^{41}$ FragFp contains more than 500 predefined binary structural fingerprints. DataWarrior calculates a similarity matrix for each compound in the data set and allocates each compound to the most similar neighbor space using the Rubberbanding force-field approach. $^{41}$ Compounds with a structural similarity $>0.95$ were clustered for the analysis.

SPR Inhibition in Solution Assay (ISA). The SPR ISA was performed in analogy to the protocol developed by Chen et al. ${ }^{30}$ with the following differences. Instead of a biotinylated peptide, a lysinetagged version of the Nrf2-peptide (KKKKAFFAQLQLDEETGEFL) was utilized for tethering to the sensor surface. For the covalent tethering, a CM5 biosensor (GE Healthcare, research grade) was employed using HBS-P [10 mM HEPES, $150 \mathrm{mM} \mathrm{NaCl}, 0.05 \%$ (v/v) Tween 20, $1.0 \mathrm{mM}$ TCEP, $\mathrm{pH} 7.40$ ] as the continuous flow buffer at a rate of $10 \mu \mathrm{L} / \mathrm{min}$ at $20^{\circ} \mathrm{C}$ on a BIAcore 3000 optical biosensor unit (GE Healthcare). The surface was activated by injecting a mixture of 1 ethyl-3-(3-dimethylaminopropyl)-carbodiimide hydrochloride (EDC) and $\mathrm{N}$-hydroxysuccinimide (NHS) for $7 \mathrm{~min}$, followed by an injection of $50 \mu \mathrm{M}$ Nrf2-peptide in $10 \mathrm{mM}$ Na-acetate, $\mathrm{pH} 4.0$ for $2 \mathrm{~min}$. Any reactive groups still present on the surface were deactivated by a $7 \mathrm{~min}$ injection of $0.1 \mathrm{M}$ ethanolamine hydrochloride- $\mathrm{NaOH}, \mathrm{pH}$ 8.5. Peptide immobilization levels were typically between 400 and $600 \mathrm{RU}$ to ensure mass transport limitation and thus a protein-concentration-dependent response.

For the detection of active compounds, a solution of $25 \mathrm{nM}$ Keap 1 (Kelch domain = aa321-aa609) was prepared in running buffer and preincubated with compounds at either constant or varying concentrations. These mixtures were subsequently injected over the peptide-modified biosensor, and control samples devoid of compounds were used to determine the remaining free protein concentration of Keap 1 in response to compound binding. For this, the initial association slopes of the binding sensorgrams have been measured (interval 5-15 s after sample injection) for each sample, followed by a regeneration of the sensor for the next cycle through a $45 \mathrm{~s}$ injection of $50 \mathrm{mM} \mathrm{Tris} / \mathrm{Cl}$, $0.25 \%$ SDS, $5 \mathrm{mM}$ TCEP, $\mathrm{pH} 7.5$. Results have been reported as $K_{\mathrm{D}^{-}}$ values for concentration-response experiments. This was achieved by a nonlinear regression analysis of the concentration-response data, which is normalized by the control samples representing $0 \%$ inhibition.

SPR Direct Binding Assay (DBA). The SPR DBA was performed as described previously, ${ }^{31}$ with the difference that HBS-P $[10 \mathrm{mM}$ HEPES, $150 \mathrm{mM} \mathrm{NaCl}, 0.05 \%$ (v/v) Tween 20, $1.0 \mathrm{mM}$ TCEP, $\mathrm{pH}$ 7.40] was used as a continuous flow buffer and that the contact and dissociation times were 45 and $60 \mathrm{~s}$, respectively.

Isothermal Titration Calorimetry (ITC). Isothermal titration calorimetry experiments were carried out on a MicroCal ITC-200 system (GE Healthcare) using Keap1 protein (Kelch domain = aa321aa609) that had been passed through a PD-10 column (GE Healthcare) equilibrated with $10 \mathrm{mM}$ HEPES, $150 \mathrm{mM} \mathrm{NaCl}, 0.05 \%(\mathrm{v} / \mathrm{v}$ ) Tween 20, $1 \mathrm{mM}$ TCEP, $\mathrm{pH} 7.40$, and 1\% DMSO. Complete titration of $60 \mu \mathrm{M}$ Keap 1 was typically achieved by injecting $19 \times 2 \mu \mathrm{L}$ of aliquots of a 0.6 $\mathrm{mM}$ compound at a temperature of $25^{\circ} \mathrm{C}$ and a spacing interval of $90 \mathrm{~s}$ between injections. The thermodynamic binding parameters were extracted by nonlinear regression analysis of the binding isotherms (Microcal Origin version 7.0 software package). A single site binding model was applied, yielding binding enthalpy $(\Delta H)$, stoichiometry $(n)$, entropy $(\Delta S)$, and association constant $\left(K_{\mathrm{a}}\right)$.

Aqueous Solubility. The aqueous solubility of compound 14 was determined at AstraZeneca as reported previously. ${ }^{62}$

Cell Permeability. The efflux-inhibited permeability of compound 14 across Caco- 2 cell monolayers was determined in the presence of a cocktail of inhibitors of efflux transporters $(50 \mu \mathrm{M}$ quinidine, $30 \mu \mathrm{M}$ benzbromarone, and $20 \mu \mathrm{M}$ sulfasalazine) by the DMPK department at Pharmaron as reported previously. ${ }^{63}$

Clearance by Human Liver Microsomes. The clearance of compound 14 by human liver microsomes $\left(\mathrm{CL}_{\text {int }}\right)$ was determined at AstraZeneca as reported previously. ${ }^{62}$

Cell Assay for 14. The cellular activity of macrocycle 14 was determined by DiscoveRx using their PathHunter U2OS Keap1-Nrf2 functional assay, ${ }^{64}$ which quantifies the translocation of Nrf2 into the nucleus due to inhibition of formation of the Keap1-Nrf2 complex.

Keap1 Expression, Purification, and Crystallization with 14. The DNA coding domain for human Keap $1(6 \times$ His- TCS-Keap 1 (A321-T609) [E540A, E542S]) was cloned into pET24 and expressed in Escherichia coli BL21(DE3)-Star in LB media at $291 \mathrm{~K}$. After harvest, the cells were resuspended in $20 \mathrm{mM}$ Tris $/ \mathrm{HCl}, \mathrm{pH} 7.5,200 \mathrm{mM} \mathrm{NaCl}$, $5 \%$ glycerol, $1 \mathrm{mM}$ DTT, protease inhibitors, disrupted with a highpressure homogenizer, and clarified by centrifugation. Purification was made by affinity chromatography using Ni Sepharose FF (GE Healthcare), eluted in one step with $300 \mathrm{mM}$ imidazole followed by SEC (Superdex200, GE Healthcare). The purified protein was detagged at room temperature with a thrombin cleave kit (Sigma) and finalized by a second SEC step (Superdex200, GE Healthcare) in buffer $20 \mathrm{mM}$ Tris/ $\mathrm{HCl}, \mathrm{pH}$ 7.5, $5 \mathrm{mM}$ DTT before concentration (Amicon cells, $10 \mathrm{kDa}$ cutoff). Crystals were grown at $20^{\circ} \mathrm{C}$ in sitting drops using $200 \mathrm{~nL}$ of protein $(11-13 \mathrm{mg} / \mathrm{mL})$ and $200 \mathrm{~nL}$ of well solution (3.7-4.1 M ammonium acetate, $0.09 \mathrm{M}$ sodium acetate $\mathrm{pH}$ 4.6 , and $10 \mathrm{mM}$ cadmium chloride). Crystals appeared after one day but continued to grow for approximately one week. Compound 14 was 
soaked by incubating crystals for $1 \mathrm{~h}$ with $1 \mathrm{mM}$ compound in $5 \mathrm{M}$ ammonium acetate and $0.1 \mathrm{M}$ sodium acetate at $\mathrm{pH}$ 4.6. Crystals were subsequently frozen in liquid nitrogen using a soaking solution supplemented with $20 \%$ glycerol as the cryoprotectant prior to data collection.

Data Collection, Structure Solution, and Refinement. X-ray diffraction data was collected at the European Synchrotron Facility, beamline ID29, using a Pilatus 6M-F pixel detector to a resolution of $2.37 \AA$. The data were indexed and integrated with MOSFLM ${ }^{65}$ and scaled with SCALA ${ }^{66}$ in the space group $P 2_{1} 2_{1} 2_{1}$ with cell dimensions of 75.775 .8203 .1 A. Two Keap1 molecules were identified by PHASER ${ }^{67}$ using a published Keap1 structure (PDB ID: $1 Z^{2} \mathrm{GK}^{25}$ ) as a search model. The Keap1 macrocycle complex was further refined by alternative cycles of model rebuilding in $\operatorname{Coot}^{68}$ and refinement in AutoBuster 2.11.6 (Global Phasing Ltd, Cambridge U.K.). A final model was refined to an R/Rfree of $19 / 21 \%$. Full data collection and refinement statistics can be found in Table S5, and the $2 \mathrm{Fo}-\mathrm{Fc}$ electron density of compound $\mathbf{1 4}$ is in Figure S8. The coordinates and corresponding structure factors have been deposited to the Protein Data Bank with accession code 6Z6A.

\section{ASSOCIATED CONTENT}

\section{(s) Supporting Information}

The Supporting Information is available free of charge at https://pubs.acs.org/doi/10.1021/acs.jmedchem.0c01569.

Supporting tables, supporting figures, computational procedures, NMR spectra of all new compounds, purity reports for compounds 6-18 (PDF)

Structures (SMILES codes) for the sets of 41 and 217 macrocyclic cores (XLSX)

Structures (SMILES codes) and biological properties for compounds 6-18, (CSV)

\section{Accession Codes}

Coordinates and structure factors for the complex of Keap1 with compound 14 have been deposited with the PDB with accession code 6Z6A. The authors will release the atomic coordinates and experimental data upon article publication.

\section{AUTHOR INFORMATION}

\section{Corresponding Authors}

Stefan Schiesser - Department of Medicinal Chemistry, Research and Early Development, Respiratory and Immunology, BioPharmaceuticals R\&D, AstraZeneca, 43183 Mölndal, Sweden; 다잉.org/0000-0002-8668-2844; Email: stefan.schiesser@astrazeneca.com

Jan Kihlberg - Department of Chemistry - BMC, Uppsala University, 75123 Uppsala, Sweden; 10 orcid.org/00000002-4205-6040; Email: jan.kihlberg@kemi.uu.se

\section{Authors}

Fabio Begnini - Department of Chemistry - BMC, Uppsala University, 75123 Uppsala, Sweden

Vasanthanathan Poongavanam - Department of Chemistry BMC, Uppsala University, 75123 Uppsala, Sweden; (ㄱ) orcid.org/0000-0002-8880-9247

Björn Over - Department of Medicinal Chemistry, Research and Early Development, Early Cardiovascular, Renal and Metabolism, BioPharmaceuticals R\&D, AstraZeneca, 43183 Mölndal, Sweden

Marie Castaldo - Discovery Biology, Discovery Sciences, RぬD, AstraZeneca, 43183 Mölndal, Sweden

Stefan Geschwindner - Structure, Biophysics \& FragmentBased Lead Generation, Discovery Sciences, R\&D,
AstraZeneca, 43183 Mölndal, Sweden; 이이.org/00000002-2154-8345

Patrik Johansson - Structure, Biophysics \& Fragment-Based Lead Generation, Discovery Sciences, R\&D, AstraZeneca, 43183 Mölndal, Sweden

Mohit Tyagi - Department of Chemistry - BMC, Uppsala University, 75123 Uppsala, Sweden

Christian Tyrchan - Department of Medicinal Chemistry, Research and Early Development, Respiratory and Immunology, BioPharmaceuticals R\&D, AstraZeneca, 43183 Mölndal, Sweden

Lisa Wissler - Structure, Biophysics \& Fragment-Based Lead Generation, Discovery Sciences, R\&D, AstraZeneca, 43183 Mölndal, Sweden

Peter Sjö - Drugs for Neglected Diseases initiative (DNDi), 1202 Geneva, Switzerland

Complete contact information is available at:

https://pubs.acs.org/10.1021/acs.jmedchem.0c01569

\section{Author Contributions}

${ }^{\#}$ F.B., V.P., and B.O. made equal contributions to the manuscript. The manuscript was written through contributions of all authors. All authors have given approval to the final version of the manuscript.

\section{Funding}

This work was funded by grants from the Swedish Research Council (grant no. 2016-05160) and AstraZeneca.

\section{Notes}

The authors declare the following competing financial interest(s): M.C., S.G., P.J., C.T., L.W., and S.S. are employees and shareholders of AstraZeneca. P.S. is an employee of Drugs for Neglected Diseases initiative (DNDi).

\section{ACKNOWLEDGMENTS}

We thank Dr. Matthew Perry at AstraZeneca for contributions to the selection of the 41 lead-like macrocycle cores and for comments on the manuscript and Dr. Werngard Czechtizky at AstraZeneca for providing valuable comments on the manuscript.

\section{ABBREVIATIONS}

bRo5, beyond the rule of $5 ; \mathrm{Cl}_{\text {int }}$, human microsomal metabolism; cLogS, calculated solubility; DNP, Dictionary of Natural Products; ISA, inhibition in solution assay; ITC, isothermal titration calorimetry; Keap1, Kelch-like ECH Associated Protein-1; Nrf2, nuclear factor, erythroid 2 Like 2; NRotB, number of rotatable bonds; $\mathrm{P}_{\text {app }} \mathrm{AB}+$ inh, effluxinhibited permeability across a Caco- 2 cell monolayer; PPI, protein-protein interaction; SPR, surface plasmon resonance; TCE, 2,2,2-trichloroethyl; TPSA, topological polar surface area

\section{REFERENCES}

(1) Hopkins, A. L.; Groom, C. R. The druggable genome. Nat. Rev. Drug Discovery 2002, 1, 727-730.

(2) Doak, B. C.; Zheng, J.; Dobritzsch, D.; Kihlberg, J. How beyond rule of 5 drugs and clinical candidates bind to their targets. J. Med. Chem. 2016, 59, 2312-2327.

(3) Egbert, M.; Whitty, A.; Keserü, G. M.; Vajda, S. Why some targets benefit from beyond rule of five drugs. J. Med. Chem. 2019, 62, 1000510025 .

(4) Surade, S.; Blundell, T. L. Structural biology and drug discovery of difficult targets: the limits of ligandability. Chem. Biol. 2012, 19, 42-50. 
(5) Doak, B. C.; Over, B.; Giordanetto, F.; Kihlberg, J. Oral druggable space beyond the rule of 5 : Insights from drugs and clinical candidates. Chem. Biol. 2014, 21, 1115-1142.

(6) DeGoey, D. A.; Chen, H.-J.; Cox, P. B.; Wendt, M. D. Beyond the rule of 5: lessons learned from AbbVie's drugs and compound collection. J. Med. Chem. 2018, 61, 2636-2651.

(7) Herein, we have used the common definition of a macrocycle, i.e. a compound that has 12 or more atoms in a ring.

(8) Villar, E. A.; Beglov, D.; Chennamadhavuni, S.; Porco, J. A.; Kozakov, D.; Vajda, S.; Whitty, A. How proteins bind macrocycles. Nat. Chem. Biol. 2014, 10, 723-732.

(9) Mallinson, J.; Collins, I. Macrocycles in new drug discovery. Future Med. Chem. 2012, 4, 1409-1438.

(10) Blum, L. C.; Reymond, J.-L. 970 Million druglike small molecules for virtual screening in the chemical universe database GDB-13. J. Am. Chem. Soc. 2009, 131, 8732-8733.

(11) Newman, D. J.; Cragg, G. M. Natural products as sources of new drugs from 1981 to 2014. J. Nat. Prod. 2016, 79, 629-661.

(12) Over, B.; Wetzel, S.; Grütter, C.; Nakai, Y.; Renner, S.; Rauh, D.; Waldmann, H. Natural-product-derived fragments for fragment-based ligand discovery. Nat. Chem. 2013, 5, 21-28.

(13) Erlanson, D. A.; Fesik, S. W.; Hubbard, R. E.; Jahnke, W.; Jhoti, $\mathrm{H}$. Twenty years on: the impact of fragments on drug discovery. Nat. Rev. Drug Discovery 2016, 15, 605-619.

(14) Dictionary of Natural Products; CRC Press, Taylor \& Francis: Boca Raton, 2020.

(15) Bemis, G. W.; Murcko, M. A. The properties of known drugs. 1. Molecular frameworks. J. Med. Chem. 1996, 39, 2887-2893.

(16) Wishart, D. S.; Feunang, Y. D.; Guo, A. C.; Lo, E. J.; Marcu, A.; Grant, J. R.; Sajed, T.; Johnson, D.; Li, C.; Sayeeda, Z.; Assempour, N.; Iynkkaran, I.; Liu, Y.; Maciejewski, A.; Gale, N.; Wilson, A.; Chin, L.; Cummings, R.; Le, D.; Pon, A.; Knox, C.; Wilson, M. DrugBank 5.0: a major update to the DrugBank database for 2018. Nucleic Acids Res. 2018, 46, D1074-D1082.

(17) Cuadrado, A.; Rojo, A. I.; Wells, G.; Hayes, J. D.; Cousin, S. P.; Rumsey, W. L.; Attucks, O. C.; Franklin, S.; Levonen, A.-L.; Kensler, T. W.; Dinkova-Kostova, A. T. Therapeutic targeting of the NRF2 and KEAP1 partnership in chronic diseases. Nat. Rev. Drug Discovery 2019, 18, 295-317.

(18) Jiang, Z.-Y.; Lu, M.-C.; You, Q.-D. Discovery and development of Kelch-like ECH-associated protein 1. Nuclear factor erythroid 2-related factor 2 (KEAP1:NRF2) protein-protein interaction inhibitors: achievements, challenges, and future directions. J. Med. Chem. 2016, 59, 10837-10858.

(19) Pallesen, J. S.; Tran, K. T.; Bach, A. Non-covalent small-molecule Kelch-like ECH-associated protein 1-nuclear factor erythroid 2related factor 2 (Keap1-Nrf2) inhibitors and their potential for targeting central nervous system diseases. J. Med. Chem. 2018, 61, $8088-8103$.

(20) Tran, K. T.; Pallesen, J. S.; Solbak, S. M. Ø.; Narayanan, D.; Baig, A.; Zang, J.; Aguayo-Orozco, A.; Carmona, R. M. C.; Garcia, A. D.; Bach, A. A comparative assessment study of known small-molecule Keap1-Nrf2 protein-protein interaction inhibitors: chemical synthesis, binding properties, and cellular activity. J. Med. Chem. 2019, 62, $8028-8052$.

(21) Zhong, M.; Lynch, A.; Muellers, S. N.; Jehle, S.; Luo, L.; Hall, D. R.; Iwase, R.; Carolan, J. P.; Egbert, M.; Wakefield, A.; Streu, K.; Harvey, C. M.; Ortet, P. C.; Kozakov, D.; Vajda, S.; Allen, K. N.; Whitty, A. Interaction energetics and druggability of the protein-protein interaction between Kelch-like ECH-Associated Protein 1 (KEAP1) and Nuclear Factor Erythroid 2 Like 2 (Nrf2). Biochemistry 2020, 59, $563-581$.

(22) Hajduk, P. J.; Huth, J. R.; Fesik, S. W. Druggability indices for protein targets derived from NMR-based screening data. J. Med. Chem. 2005, 48, 2518-2525.

(23) Kozakov, D.; Hall, D. R.; Napoleon, R. L.; Yueh, C.; Whitty, A.; Vajda, S. New frontiers in druggability. J. Med. Chem. 2015, 58, 90639088.
(24) Marcotte, D.; Zeng, W.; Hus, J.-C.; McKenzie, A.; Hession, C.; Jin, P.; Bergeron, C.; Lugovskoy, A.; Enyedy, I.; Cuervo, H.; Wanga, D.; Atmanene, C.; Roecklin, D.; Vecchi, M.; Vivat, V.; Kraemer, J.; Winkler, D.; Hong, V.; Chao, J.; Lukashev, M.; Silvian, L. Small molecules inhibit the interaction of Nrf2 and the Keap1 Kelch domain through a noncovalent mechanism. Bioorg. Med. Chem. 2013, 21, 4011-4019.

(25) Beamer, L. J.; Li, X.; Bottoms, C. A.; Hanninka, M. Conserved solvent and side-chain interactions in the $1.35 \AA$ structure of the Kelch domain of Keap1. Acta Crystallogr., Sect. D: Biol. Crystallogr. 2005, 61, $1335-1342$.

(26) Halgren, T. A.; Murphy, R. B.; Friesner, R. A.; Beard, H. S.; Frye, L. L.; Pollard, W. T.; Banks, J. L. Glide: A new approach for rapid, accurate docking and scoring. 2. Enrichment factors in database screening. J. Med. Chem. 2004, 47, 1750-1759.

(27) Cribiú, R.; Jäger, C.; Nevado, C. Syntheses and biological evaluation of iriomoteolide $3 \mathrm{a}$ and analogues. Angew. Chem., Int. Ed. 2009, 48, 8780-8783.

(28) Boger, D. L.; Zhou, J. Total synthesis of (+)-piperazinomycin. J. Am. Chem. Soc. 1993, 115, 11426-11433.

(29) Götschi, E.; Jenny, C.-J.; Reindl, P.; Ricklin, F. Total synthesis of cyclothialidine. Helv. Chim. Acta 1996, 79, 2219-2234.

(30) Chen, Y.; Inoyama, D.; Kong, A.-N. T.; Beamer, L. J.; Hu, L. Kinetic analyses of Keap1-Nrf2 interaction and determination of the minimal Nrf2 peptide sequence required for Keap1 binding using surface plasmon resonance. Chem. Biol. Drug Des. 2011, 78, 10141021.

(31) Jiang, C.-H.; Zhuang, C.-L.; Zhu, K.; Zhang, J.; Muehlmann, L. A.; Longo, J. P. F.; Azevedo, R. B.; Zhang, W.; Meng, N.; Zhang, H. Identification of a novel small-molecule Keap1-Nrf2 PPI inhibitor with cytoprotective effects on LPS-induced cardiomyopathy. J. Enzyme Inhib. Med. Chem. 2018, 33, 833-841.

(32) Padmanabhan, B.; Tong, K. I.; Ohta, T.; Nakamura, Y.; Scharlock, M.; Ohtsuji, M.; Kang, M.-I.; Kobayashi, A.; Yokoyama, S.; Yamamoto, M. Structural basis for defects of Keap1 activity provoked by its point mutations in lung cancer. Mol. Cell 2006, 21, 689-700.

(33) Davies, T. G.; Wixted, W. E.; Coyle, J. E.; Griffiths-Jones, C.; Hearn, K.; McMenamin, R.; Norton, D.; Rich, S. J.; Richardson, C.; Saxty, G.; Willems, H. M. G.; Woolford, A. J.-A.; Cottom, J. E.; Kou, J.P.; Yonchuk, J. G.; Feldser, H. G.; Sanchez, Y.; Foley, J. P.; Bolognese, B. J.; Logan, G.; Podolin, P. L.; Yan, H.; Callahan, J. F.; Heightman, T. D.; Kerns, J. K. Monoacidic inhibitors of the Kelch-like ECH-Associated Protein 1: Nuclear Factor Erythroid 2-Related Factor 2 (KEAP1:NRF2) protein-protein interaction with high cell potency identified by fragment-based discovery. J. Med. Chem. 2016, 59, 39914006.

(34) Greenidge, P. A.; Kramer, C.; Mozziconacci, J.-C.; Wolf, R. M. MM/GBSA binding energy prediction on the PDBbind data set: successes, failures, and directions for further improvement. J. Chem. Inf. Model. 2013, 53, 201-209.

(35) Genheden, S.; Ryde, U. The MM/PBSA and MM/GBSA methods to estimate ligand-binding affinities. Expert Opin. Drug Discovery 2015, 10, 449-461.

(36) Taylor, R.; Kennard, O. Crystallographic evidence for the existence of $\mathrm{C}-\mathrm{H} \cdots \mathrm{O}, \mathrm{C}-\mathrm{H} \cdots \mathrm{N}$, and $\mathrm{C}-\mathrm{H} \cdots \mathrm{Cl}$ hydrogen bonds. J. Am. Chem. Soc. 1982, 104, 5063-5070.

(37) Ajitha, M. J.; Huang, K.-W. Non-classical C-H $\cdots$ X hydrogen bonding and its role in asymmetric organocatalysis. Synthesis 2016, 48, 3449-3458.

(38) https://pubchem.ncbi.nlm.nih.gov/\#query=keap $1 \&$ tab= bioassay, accessed June 16, 2020.

(39) Baell, J. B.; Holloway, G. A. New substructure filters for removal of pan assay interference compounds (PAINS) from screening libraries and for their exclusion in bioassays. J. Med. Chem. 2010, 53, 27192740.

(40) Bajusz, D.; Rácz, A.; Héberger, K. Why is Tanimoto index an appropriate choice for fingerprint-based similarity calculations? J. Cheminf. 2015, 7, No. 20. 
(41) Sander, T.; Freyss, J.; von Korff, M.; Rufener, C. DataWarrior: an open-source program for chemistry aware data visualization and analysis. J. Chem. Inf. Model. 2015, 55, 460-473.

(42) Scott, D. E.; Bayly, A. R.; Abell, C.; Skidmore, J. Small molecules, big targets: drug discovery faces the protein-protein interaction challenge. Nat. Rev. Drug Discovery 2016, 15, 533-550.

(43) Lyu, J.; Wang, S.; Balius, T. E.; Singh, I.; Levit, A.; Moroz, Y. S.; O'Meara, M. J.; Che, T.; Algaa, E.; Tolmachova, K.; Tolmachev, A. A.; Shoichet, B. K.; Roth, B. L.; Irwin, J. J. Ultra-large library docking for discovering new chemotypes. Nature 2019, 566, 224-229.

(44) Gorgulla, C.; Boeszoermenyi, A.; Wang, Z.-F.; Fischer, P. D.; Coote, P. W.; Padmanabha Das, K. M.; Malets, Y. S.; Radchenko, D. S.; Moroz, Y. S.; Scott, D. A.; Fackeldey, K.; Hoffmann, M.; Iavniuk, I.; Wagner, G.; Arthanari, H. An open-source drug discovery platform enables ultra-large virtual screens. Nature 2020, 580, 663-668.

(45) Heightman, T. D.; Callahan, J. F.; Chiarparin, E.; Coyle, J. E.; Griffiths-Jones, C.; Lakdawala, A. S.; McMenamin, R.; Mortenson, P. N.; Norton, D.; Peakman, T. M.; Rich, S. J.; Richardson, C.; Rumsey, W. L.; Sanchez, Y.; Saxty, G.; Willems, H. M. G.; Wolfe, L., III; Woolford, A. J.A.; Wu, Z.; Yan, H.; Kerns, J. K.; Davies, T. G. Structure-activity and structure-conformation relationships of aryl propionic acid inhibitors of the Kelch-like ECH-Associated Protein 1/ Nuclear Factor Erythroid 2-Related Factor 2 (KEAP1/NRF2) protein-protein interaction. J. Med. Chem. 2019, 62, 4683-4702.

(46) Nielsen, T. E.; Schreiber, S. L. Towards the optimal screening collection: a synthesis strategy. Angew. Chem., Int. Ed. 2008, 47, 48-56.

(47) Karageorgis, G.; Foley, D. J.; Laraia, L.; Waldmann, H. Principle and design of pseudo-natural products. Nat. Chem. 2020, 12, 227-235.

(48) Broo, A.; Gottfries, J.; Kossenjans, M.; Lanna, L.; LindstedtAlstermark, E. L.; Nilsson, K. A.; Ohlsson, B.; Thorstensson, M. 2Substituted-3-phenylpropionic Acid Derivatives as Peroxisome Proliferator Activated Receptor Modulators and their Preparation, Pharmaceutical Compositions and Use in the Treatment of Inflammatory Bowel Disease. WO2008/108735A1.

(49) Molecular Operating Environment (MOE), 2015.10; Chemical Computing Group ULC: 1010 Sherbooke St. West, Suite \#910, Montreal, QC, Canada, H3A 2R7, 2016.

(50) Labute, P. A widely applicable set of descriptors. J. Mol. Graphics Modell. 2000, 18, 464-477.

(51) SIMCA (version 14.1) Umetrics, Umeå, Sweden. http://www. umetrics.com.

(52) Hassan, M.; Brown, R. D.; Varma-O’Brien, S.; Rogers, D. Cheminformatics analysis and learning in a data pipelining environment. Mol. Diversity 2006, 10, 283-299.

(53) Sastry, G. M.; Adzhigirey, M.; Day, T.; Annabhimoju, R.; Sherman, W. Protein and ligand preparation: parameters, protocols, and influence on virtual screening enrichments. J. Comput.-Aided Mol. Des. 2013, 27, 221-234.

(54) Schrödinger Release 2019-1: Protein Preparation Wizard; Epik, Schrödinger, LLC, New York, NY, 2019; Impact, Schrödinger, LLC, New York, NY, 2019; Prime, Schrödinger, LLC: New York, NY, 2019.

(55) Li, H.; Robertson, A. D.; Jensen, J. H. Very fast empirical prediction and rationalization of protein $\mathrm{p} K_{\mathrm{a}}$ values. Proteins 2005, 61, 704-721.

(56) Harder, E.; Damm, W.; Maple, J.; Wu, C.; Reboul, M.; Xiang, J. Y.; Wang, L.; Lupyan, D.; Dahlgren, M. K.; Knight, J. L.; Kaus, J. W.; Cerutti, D. S.; Krilov, G.; Jorgensen, W. L.; Abel, R.; Friesner, R. A. OPLS3: a force field providing broad coverage of drug-like small molecules and proteins. J. Chem. Theory Comput. 2016, 12, 281-296.

(57) Shelley, J. C.; Cholleti, A.; Frye, L. L.; Greenwood, J. R.; Timlin, M. R.; Uchimaya, M. Epik: a software program for $\mathrm{p} K_{\mathrm{a}}$ prediction and protonation state generation for drug-like molecules. J. Comput.-Aided Mol. Des. 2007, 21, 681-691.

(58) Sherman, W.; Day, T.; Jacobson, M. P.; Friesner, R. A.; Farid, R. Novel procedure for modeling ligand/receptor induced fit effects. J. Med. Chem. 2006, 49, 534-553.

(59) Schrödinger Release 2019-1: Induced Fit Docking protocol; Glide, Schrödinger, LLC, New York, NY, 2019; Prime, Schrödinger, LLC: New York, NY, 2019.
(60) Schrödinger Release 2019-1: Maestro; Schrödinger, LLC: New York, NY, 2019.

(61) Schrödinger Release 2019-1: Canvas; Schrödinger, LLC: New York, NY, 2019.

(62) Wernevik, J.; Bergström, F.; Novén, A.; Hulthe, J.; Fredlund, L.; Addison, D.; Holmgren, J.; Strömstedt, P.-E.; Rehnström, E.; Lundbäck, T. A fully integrated assay panel for early drug metabolism and pharmacokinetics profiling. Assay Drug Dev. Technol. 2020, 18, 157-179.

(63) Fredlund, L.; Winiwarter, S.; Hilgendorf, C. In vitro intrinsic permeability: A transporter-independent measure of Caco-2 cell permeability in drug design and development. Mol. Pharmaceutics 2017, 14, 1601-1609.

(64) DiscoveRx. PathHunter Keap1-Nrf2 Functional Assay for chemiluminescent detection of activated Nrf2 Product Booklet: 93 $0821 \mathrm{C} 3$.

(65) Battye, T. G.; Kontogiannis, L.; Johnson, O.; Powell, H. R.; Leslie, A. G. iMOSFLM: a new graphical interface for diffraction-image processing with MOSFLM. Acta Crystallogr., Sect. D: Biol. Crystallogr. 2011, 67, 271-281.

(66) Evans, P. Scaling and assessment of data quality. Acta Crystallogr., Sect. D: Biol. Crystallogr. 2006, 62, 72-82.

(67) McCoy, A. J.; Grosse-Kunstleve, R. W.; Adams, P. D.; Winn, M. D.; Storoni, L. C.; Read, R. J. Phaser crystallographic software. J. Appl. Crystallogr. 2007, 40, 658-674.

(68) Emsley, P.; Cowtan, K. Coot: model-building tools for molecular graphics. Acta Crystallogr., Sect. D: Biol. Crystallogr. 2004, 60, 21262132. 